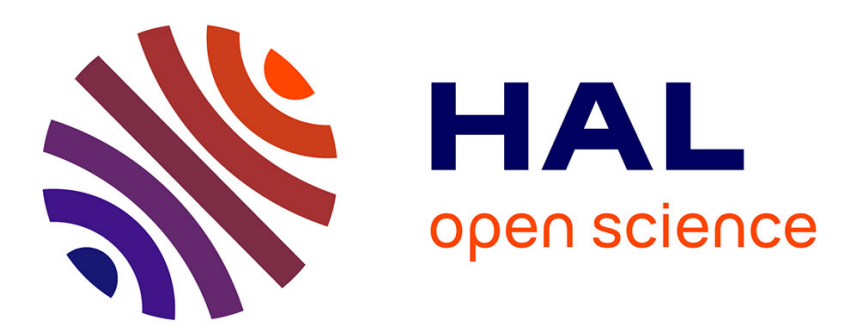

\title{
Convergence and error analysis for a class of splitting schemes in incompressible fluid-structure interaction
}

Miguel Angel Fernández, Jimmy Mullaert

\section{To cite this version:}

Miguel Angel Fernández, Jimmy Mullaert. Convergence and error analysis for a class of splitting schemes in incompressible fluid-structure interaction. IMA Journal of Numerical Analysis, 2016, 36 (4), pp.1748-1782. 10.1093/imanum/drv055 . hal-01102975v3

\section{HAL Id: hal-01102975 \\ https://hal.inria.fr/hal-01102975v3}

Submitted on 23 Oct 2015

HAL is a multi-disciplinary open access archive for the deposit and dissemination of scientific research documents, whether they are published or not. The documents may come from teaching and research institutions in France or abroad, or from public or private research centers.
L'archive ouverte pluridisciplinaire HAL, est destinée au dépôt et à la diffusion de documents scientifiques de niveau recherche, publiés ou non, émanant des établissements d'enseignement et de recherche français ou étrangers, des laboratoires publics ou privés. 
Convergence and error analysis for a class of splitting schemes in incompressible fluid-structure interaction

Miguel A. Fernández, Jimmy Mullaert

RESEARCH REPORT

$\mathrm{N}^{\circ} 8670$

January 2015

Project-Team Reo 



\title{
Guntas
}

\section{Convergence and error analysis for a class of splitting schemes in incompressible fluid-structure interaction}

\author{
Miguel A. Fernández ${ }^{* \dagger}$, Jimmy Mullaert*† \\ Project-Team Reo \\ Research Report $\mathrm{n}^{\circ} 8670$ - January 2015 - 36 pages
}

\begin{abstract}
This paper is devoted to the convergence analysis of the generalized Robin-Neumann schemes introduced in [Internat. J. Numer. Methods Engrg., 101(3):199-229, 2015] for the coupling of a viscous incompressible fluid with a thick-walled elastic or viscoelastic structure. To this purpose, a representative linearized setting is considered. The methods are formulated within a class of operator splitting schemes which treat implicitly the coupling between the fluid and the solid inertia contributions. This guarantees energy stability. A priori error estimates are derived for all the explicit and semi-implicit variants. The analysis predicts a non-uniformity in space of the splitting error, hence confirming the numerical evidence of [Internat. J. Numer. Methods Engrg., 101(3):199-229, 2015] for the explicit variants. Besides, the analysis demonstrates that the genesis of this accuracy loss is the spatial non-uniformity of the discrete elastic or viscoelastic solid operator. The theoretical findings are illustrated via a numerical study which shows, in particular, that alternative splitting schemes recently reported in the literature also suffer from these accuracy issues.
\end{abstract}

Key-words: fluid-structure interaction, Stokes flow, visco-elastic solid, finite element method, mass-lumping, splitting schemes, a priori error estimates.

This work was supported by the French National Research Agency (ANR) through the EXIFSI project (ANR-12-JS01-0004).

* Inria, REO project-team, Rocquencourt - B.P. 105, F-78153 Le Chesnay cedex, France

$\dagger$ UPMC Univ Paris VI, REO project-team, UMR 7958 LJLL, F-75005 Paris, France

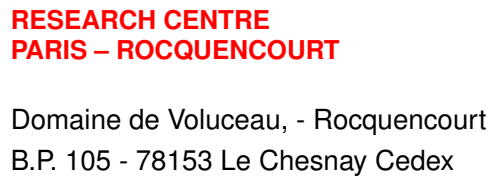




\section{Analyse de convergence et d'erreur pour une classe de méthodes de découplage en interaction fluide-structure}

Résumé : Cet article est consacré à l'analyse de la convergence des schémas Robin-Neumann généralisés introduits dans [Internat. J. Numer. Methods Engrg., 101(3):199-229, 2015] pour le couplage d'un fluide visqueux incompressible avec une structure élastique épaisse. Les méthodes sont formulées dans une classe de schémas à pas fractionnaire qui traitent implicitement le couplage entre le fluide et l'inertie du solide. Ceci garantit la stabilité dans la norme de l'énergie. Des estimations d'erreur a priori sont obtenues pour toutes les variantes explicites et semiimplicites. L'analyse prévoit une non-uniformité en espace de l'erreur de découplage, confirmant ainsi les résultats numériques présentés dans [Internat. J. Numer. Methods Engrg., 101(3):199229, 2015] pour les variantes explicites. En outre, l'analyse montre que l'origine de cette perte de précision est la non-uniformité spatiale de l'opérateur solide visco-élastique discret. Les résultats théoriques sont illustrés par une étude numérique qui montre, en particulier, que des schémas alternatifs récemment proposés dans la littérature ont le même type de problèmes de précision.

Mots-clés : interaction fluide-structure, écoulement de Stokes, structure visco-élastique, méthode des éléments finis, condensation de masse, schémas de découplage en temps, estimations d'erreur a priori. 


\section{Introduction}

This paper deals with the numerical approximation of fluid-structure interaction systems involving a viscous incompressible fluid and a thick-walled structure. We consider a coupled system in which the fluid is described by the Stokes equations, in a fixed configuration, and the solid by the linear visco-elastodynamics equations. Standard kinematic and kinetic coupling conditions are enforced on the interface. Though simplified, this linear setting retains some of the main numerical issues that appear in the simulation of complex non-linear incompressible fluid-structure systems (see, e.g., [13, 23]).

The key feature of the numerical methods analyzed in the present work is that they provide different degrees of splitting in the computation of the fluid and the solid: semi-implicit and explicit coupling schemes. Semi-implicit coupling schemes perform an implicit-explicit splitting of the coupled problem based on a specific fractional-step time-stepping of the fluid or the solid (see, e.g., [18, 32, 3, 7]). The implicit part of the coupling guarantees numerical stability while the explicit one reduces computational complexity.

Explicit coupling schemes are those that uncouple the fluid and solid time-marchings through specific explicit discretizations of the interface conditions. This yields, a priori, the most efficient solution procedure, but the design of such (stable and accurate) methods in incompressible fluidstructure interaction is a delicate problem. The main reason is the extremely stiff nature of the Dirichlet-Neumann interface coupling: the so-called added-mass effect (see, e.g., [13, 23]). In fact, stability in explicit coupling demands a different treatment of the interface conditions.

In $[10,11]$, stability is achieved from a specific Robin-Robin formulation derived from Nitsche's interface method (see, e.g., [26]). The price to pay is a perturbed truncation error whose leading term scales as $\mathcal{O}(\tau / h)$, with $\tau, h$ respectively denoting the time and space discretization parameters. Suitable extrapolations and correction iterations are hence needed to enhance accuracy (see [11]). Alternative explicit strategies have been recently proposed in [11, Section 4.3] and in [4], but their rigorous stability analysis remains open.

In this paper we consider the explicit coupling schemes introduced in [19, 21]. The key ingredient for the stability of these methods is a certain interface Robin consistency that can be retrieved at the space semi-discrete level, using a lumped-mass approximation in the structure, and which consistently integrates the solid inertial effects within the fluid. Basically, these splitting schemes extend the Robin-Neumann explicit coupling paradigm of [17, 20] for thinwalled structures, to the case of the coupling with a thick-walled structure. Extensive numerical evidence reported in [21] indicates, however, that the optimal accuracy of the thin-walled solid case is not preserved. More precisely, the error induced by the splitting is not uniform in $h$ and appears to be $\mathcal{O}\left(\tau^{2^{r-1}} / h^{\frac{1}{2}}\right)$, with $r \in\{0,1,2\}$ denoting the order of the explicit extrapolations.

The aim of the present work is to identify the source of this $h^{\frac{1}{2}}$ accuracy loss. To this purpose, the schemes are formulated within a wider class of operator splitting schemes in which the solid mass-lumping approximation becomes optional and simply determines the semi-implicit or explicit nature of the methods (Algorithms 1 and 2). The resulting semi-implicit schemes have some connections with the methods recently reported in [7] and present several advantages with respect to them. Error estimates are derived for all the (explicit and semi-implicit) variants using energy arguments. The analysis confirms the $\mathcal{O}\left(\tau^{2^{r-1}} / h^{\frac{1}{2}}\right)$ convergence rate observed numerically in [21]. Furthermore, it demonstrates that the $h^{\frac{1}{2}}$-loss is exclusively due the non-uniformity of the solid discrete elastic or viscoelastic operator, regardless of the mass-lumping approximation in the solid. These theoretical findings are further illustrated via numerical experiments which show, in particular, that semi-implicit scheme of [7] also suffers from this non-uniformity issue.

The numerical analysis of linear incompressible fluid-structure interaction problems has been the topic of a number of studies in the literature (see $[29,16,1,17,20])$. To the best of our 
knowledge, this is the first time that the convergence analysis addresses the case of the coupling with a thick-walled solid via explicit coupling schemes.

The rest of the paper is organized as follows. Section 2 presents the linear continuous setting which serves as model coupled problem. Section 3 introduces the space and time discretizations and the different splitting methods. We recall the stability result for the explicit schemes and show that this holds also for the new semi-implicit variants. Section 4 is devoted to the error analysis of the methods using energy arguments. The numerical experiments are reported and discussed in Section 5. Finally, a summary of the results is given in Section 6.

\section{Problem setting}

We consider a fluid-structure system described by the Stokes equations in a domain $\Omega^{\mathrm{f}} \subset \mathbb{R}^{d}$ and by the linear viscoelasticity equations in an adjacent domain $\Omega^{\mathrm{s}} \subset \mathbb{R}^{d}(d=2$ or 3$)$. We denote by $\Sigma \stackrel{\text { def }}{=} \partial \Omega^{\mathrm{s}} \cap \partial \Omega^{\mathrm{f}}$ the fluid-structure interface. We assume that $\partial \Omega^{\mathrm{f}}=\Gamma^{\mathrm{f}, \mathrm{d}} \cup \Gamma^{\mathrm{f}, \mathrm{n}} \cup \Sigma$ and $\partial \Omega^{\mathrm{s}}=\Gamma^{\mathrm{s}, \mathrm{d}} \cup \Gamma^{\mathrm{s}, \mathrm{n}} \cup \Sigma$ are given partitions of the fluid and solid boundaries, respectively, and that $T>0$ is a given final time instant.

The considered linear coupled problem reads therefore as follows: find the fluid velocity $\boldsymbol{u}: \Omega^{\mathrm{f}} \times(0, T) \rightarrow \mathbb{R}^{d}$, the fluid pressure $p: \Omega^{\mathrm{f}} \times(0, T) \rightarrow \mathbb{R}$, the structure displacement $\boldsymbol{d}: \Omega^{\mathrm{s}} \times(0, T) \rightarrow \mathbb{R}^{d}$ and the structure velocity $\dot{\boldsymbol{d}}: \Omega^{\mathrm{s}} \times(0, T) \rightarrow \mathbb{R}^{d}$ such that

$$
\begin{aligned}
& \left\{\begin{array}{rll}
\rho^{\mathrm{f}} \partial_{t} \boldsymbol{u}-\operatorname{div} \boldsymbol{\sigma}^{\mathrm{f}}(\boldsymbol{u}, p)=\mathbf{0} & \text { in } & \Omega^{\mathrm{f}} \times(0, T), \\
\operatorname{div} \boldsymbol{u}=0 & \text { in } \quad & \Omega^{\mathrm{f}} \times(0, T), \\
\boldsymbol{u}=\mathbf{0} & \text { on } & \Gamma^{\mathrm{f}, \mathrm{d}} \times(0, T), \\
\boldsymbol{\sigma}^{\mathrm{f}}(\boldsymbol{u}, p) \boldsymbol{n}^{\mathrm{f}}=\boldsymbol{h} & \text { on } & \Gamma^{\mathrm{f}, \mathrm{n}} \times(0, T),
\end{array}\right. \\
& \left\{\begin{array}{rll}
\rho^{\mathrm{s}} \partial_{t} \dot{\boldsymbol{d}}-\operatorname{div} \boldsymbol{\sigma}^{\mathrm{s}}(\boldsymbol{d}, \dot{\boldsymbol{d}})+\alpha_{0} \rho^{\mathrm{s}} \dot{\boldsymbol{d}}=\mathbf{0} & \text { in } \quad \Omega^{\mathrm{s}} \times(0, T), \\
\dot{\boldsymbol{d}}=\partial_{t} \boldsymbol{d} & \text { in } \quad \Omega^{\mathrm{s}} \times(0, T), \\
\boldsymbol{d}=\mathbf{0}, \quad \alpha_{1} \dot{\boldsymbol{d}}=\mathbf{0} & \text { on } \quad \Gamma^{\mathrm{s}, \mathrm{d}} \times(0, T), \\
\boldsymbol{\sigma}^{\mathrm{s}}(\boldsymbol{d}, \dot{\boldsymbol{d}}) \boldsymbol{n}^{\mathrm{s}}=\mathbf{0} & \text { on } \quad \Gamma^{\mathrm{s}, \mathrm{n}} \times(0, T),
\end{array}\right. \\
& \left\{\begin{array}{rll}
\boldsymbol{u}=\dot{\boldsymbol{d}} & \text { on } & \Sigma \times(0, T), \\
\boldsymbol{\sigma}^{\mathrm{s}}(\boldsymbol{d}, \dot{\boldsymbol{d}}) \boldsymbol{n}^{\mathrm{s}}=-\boldsymbol{\sigma}^{\mathrm{f}}(\boldsymbol{u}, p) \boldsymbol{n}^{\mathrm{f}} & \text { on } & \Sigma \times(0, T)
\end{array}\right.
\end{aligned}
$$

and satisfying the initial conditions $\boldsymbol{u}(0)=\boldsymbol{u}_{0}, \boldsymbol{d}(0)=\boldsymbol{d}_{0}$ and $\dot{\boldsymbol{d}}(0)=\boldsymbol{v}_{0}$. Here, $\rho^{\mathrm{f}}, \rho^{\mathrm{s}}>0$ stand for the fluid and solid densities, $\boldsymbol{h}$ for a given surface traction on $\Gamma^{\mathrm{f}, \mathrm{n}}$ and $\boldsymbol{n}^{\mathrm{f}}, \boldsymbol{n}^{\mathrm{s}}$ for the exterior unit normal vectors to the boundaries of $\Omega^{\mathrm{f}}$ and $\Omega^{\mathrm{s}}$, respectively. The fluid stress tensor $\boldsymbol{\sigma}^{\mathrm{f}}(\boldsymbol{u}, p)$ is given by

$$
\boldsymbol{\sigma}^{\mathrm{f}}(\boldsymbol{u}, p) \stackrel{\text { def }}{=}-p \boldsymbol{I}_{\mathbb{R}^{d \times d}}+2 \mu \boldsymbol{\epsilon}(\boldsymbol{u}), \quad \boldsymbol{\epsilon}(\boldsymbol{u}) \stackrel{\text { def }}{=} \frac{1}{2}\left(\boldsymbol{\nabla} \boldsymbol{u}+\nabla \boldsymbol{u}^{\mathrm{T}}\right),
$$

where $\mu>0$ denotes the fluid dynamic viscosity. For the solid, the stress tensor is defined by the relations

$$
\boldsymbol{\sigma}^{\mathrm{s}}(\boldsymbol{d}, \dot{\boldsymbol{d}}) \stackrel{\text { def }}{=} \boldsymbol{\sigma}(\boldsymbol{d})+\alpha_{1} \boldsymbol{\sigma}(\dot{\boldsymbol{d}}), \quad \boldsymbol{\sigma}(\boldsymbol{d}) \stackrel{\text { def }}{=} 2 L_{1} \boldsymbol{\epsilon}(\boldsymbol{d})+L_{2}(\operatorname{div} \boldsymbol{d}) \boldsymbol{I}_{\mathbb{R}^{d \times d}},
$$

where $L_{1}, L_{2}>0$ stand for the Lamé coefficients and $\alpha_{0}, \alpha_{1} \geq 0$ for the Rayleigh damping parameters. Well-posedness results for this type of linear fluid-structure interaction problem can be found in [15]. 
Remark 1 The solid damping parameters $\alpha_{0}, \alpha_{1}$ can both be set to 0 in (2)-(3), so that the case of the coupling with a purely elastic solid is also included.

Remark 2 The linear problem (1)-(3) can be viewed as a simplified version of the non-linear coupled problems often used to describe more general fluid-structure interaction systems (see, e.g., [21, Sections 5-6]). Yet it retains some of the main numerical issues that appear in the non-linear framework.

In what follows, we will make use of the functional spaces

$$
\begin{array}{ll}
\boldsymbol{V}^{\mathrm{f}} \stackrel{\text { def }}{=}\left\{\boldsymbol{v}^{\mathrm{s}} \in\left[H^{1}\left(\Omega^{\mathrm{f}}\right)\right]^{d} /\left.\boldsymbol{v}^{\mathrm{f}}\right|_{\Gamma^{\mathrm{f}, \mathrm{d}}}=\mathbf{0}\right\}, & Q \stackrel{\text { def }}{=} L^{2}\left(\Omega^{\mathrm{f}}\right), \\
\boldsymbol{V}^{\mathrm{s}} \stackrel{\text { def }}{=}\left\{\boldsymbol{v}^{\mathrm{s}} \in\left[H^{1}\left(\Omega^{\mathrm{s}}\right)\right]^{d} /\left.\boldsymbol{v}^{\mathrm{s}}\right|_{\Gamma^{\mathrm{s}, \mathrm{d}}}=\mathbf{0}\right\}, & \boldsymbol{W} \stackrel{\text { def }}{=}\left\{\left(\boldsymbol{v}^{\mathrm{f}}, \boldsymbol{v}^{\mathrm{s}}\right) \in \boldsymbol{V}^{\mathrm{f}} \times \boldsymbol{V}^{\mathrm{s}} /\left.\boldsymbol{v}^{\mathrm{f}}\right|_{\Sigma}=\left.\boldsymbol{v}^{\mathrm{s}}\right|_{\Sigma}\right\}
\end{array}
$$

and the following bi-linear and linear forms:

$$
\begin{gathered}
a\left(\boldsymbol{u}, \boldsymbol{v}^{\mathrm{f}}\right) \stackrel{\text { def }}{=} 2 \mu\left(\boldsymbol{\epsilon}(\boldsymbol{u}), \boldsymbol{\epsilon}\left(\boldsymbol{v}^{\mathrm{f}}\right)\right)_{\Omega^{\mathrm{f}}}, \quad b\left(p, \boldsymbol{v}^{\mathrm{f}}\right) \stackrel{\text { def }}{=}-\left(p, \operatorname{div} \boldsymbol{v}^{\mathrm{f}}\right)_{\Omega^{\mathrm{f}}}, \quad l\left(\boldsymbol{v}^{\mathrm{f}}\right) \stackrel{\text { def }}{=}\left(\boldsymbol{h}, \boldsymbol{v}^{\mathrm{f}}\right)_{\Gamma^{\mathrm{f}, \mathrm{n}},}, \\
a^{\mathrm{e}}\left(\boldsymbol{d}, \boldsymbol{v}^{\mathrm{s}}\right) \stackrel{\text { def }}{=}\left(\boldsymbol{\sigma}(\boldsymbol{d}), \boldsymbol{\epsilon}\left(\boldsymbol{v}^{\mathrm{s}}\right)\right)_{\Omega^{\mathrm{s}}}, \quad a^{\mathrm{v}}\left(\dot{\boldsymbol{d}}, \boldsymbol{v}^{\mathrm{s}}\right) \stackrel{\text { def }}{=} \alpha_{0} \rho^{\mathrm{s}}\left(\dot{\boldsymbol{d}}, \boldsymbol{v}^{\mathrm{s}}\right)_{\Omega^{\mathrm{s}}}+\alpha_{1}\left(\boldsymbol{\sigma}(\dot{\boldsymbol{d}}), \boldsymbol{\epsilon}\left(\boldsymbol{v}^{\mathrm{s}}\right)\right)_{\Omega^{\mathrm{s}}} .
\end{gathered}
$$

Here, the symbol $(\cdot, \cdot)_{\omega}$ stands for the standard inner-product of $L^{2}(\omega)$, for a given domain $\omega$ of $\mathbb{R}^{d}$ or $\mathbb{R}^{d-1}$. We denote by $\|\cdot\|_{\mathrm{e}}$ and $\|\cdot\|_{\mathrm{v}}$ the norms associated to the inner-products $a^{\mathrm{e}}$ and $a^{\mathrm{v}}$, respectively, in particular, we have

$$
\|\dot{\boldsymbol{d}}\|_{\mathrm{v}}^{2} \stackrel{\text { def }}{=} \alpha_{0} \rho^{\mathrm{s}}\|\dot{\boldsymbol{d}}\|_{0, \Omega^{\mathrm{s}}}^{2}+\alpha_{1}\|\dot{\boldsymbol{d}}\|_{\mathrm{e}}^{2}
$$

We also set

$$
\|\boldsymbol{u}\|_{\boldsymbol{V}^{\mathrm{f}}} \stackrel{\text { def }}{=} \mu^{\frac{1}{2}}\|\boldsymbol{\nabla} \boldsymbol{u}\|_{0, \Omega^{\mathrm{f}}}, \quad\|p\|_{Q} \stackrel{\text { def }}{=} \mu^{-\frac{1}{2}}\|p\|_{0, \Omega^{\mathrm{f}}} .
$$

The coupled problem (1)-(3) admits the following variational formulation: for $t \in(0, T)$, find $(\boldsymbol{u}(t), \dot{\boldsymbol{d}}(t)) \in \boldsymbol{W}, p(t) \in Q$ and $\boldsymbol{d}(t) \in \boldsymbol{V}^{\mathrm{s}}$ such that

$$
\left\{\begin{array}{l}
\dot{\boldsymbol{d}}=\partial_{t} \boldsymbol{d}, \\
\rho^{\mathrm{f}}\left(\partial_{t} \boldsymbol{u}, \boldsymbol{v}^{\mathrm{f}}\right)_{\Omega^{\mathrm{f}}}+a\left(\boldsymbol{u}, \boldsymbol{v}^{\mathrm{f}}\right)+b\left(p, \boldsymbol{v}^{\mathrm{f}}\right)-b(q, \boldsymbol{u})+\rho^{\mathrm{s}}\left(\partial_{t} \dot{\boldsymbol{d}}, \boldsymbol{v}^{\mathrm{s}}\right)_{\Omega^{\mathrm{s}}}+a^{\mathrm{e}}\left(\boldsymbol{d}, \boldsymbol{v}^{\mathrm{s}}\right)+a^{\mathrm{v}}\left(\dot{\boldsymbol{d}}, \boldsymbol{v}^{\mathrm{s}}\right)=l\left(\boldsymbol{v}^{\mathrm{f}}\right),
\end{array}\right.
$$

for all $\left(\boldsymbol{v}^{\mathrm{f}}, \boldsymbol{v}^{\mathrm{s}}\right) \in \boldsymbol{W}$ and $q \in Q$.

\section{$3 \quad$ Numerical methods}

This section is devoted to the numerical approximation of the coupled problem (5). The key feature of the methods considered is that they provide different degrees of splitting between the computation of the fluid and the solid. We recall the family of schemes introduced in [19, 21] (Algorithm 2) and a variant (Algorithm 1) with less degree of splitting between the computation of the fluid and the solid. The error analysis of these methods (Algorithms 1 and 2) will be the topic of Section 4. Finally, we review some alternative splitting schemes that can be found in the literature (Algorithm 3, see [7]).

The spatial semi-discrete finite element approximation of the coupled problem (5) is introduced in Section 3.1. Section 3.2 presents the time discretization and the different fluid-solid time splitting schemes.

$\mathrm{RR} \mathrm{n}^{\circ} 8670$ 


\subsection{Spatial semi-discrete approximation}

We consider a finite element approximation in space based on continuous piece-wise affine functions. The corresponding finite element spaces are denoted by $\boldsymbol{V}_{h}^{\mathrm{f}} \subset \boldsymbol{V}^{\mathrm{f}}, Q_{h} \subset Q, \boldsymbol{V}_{h}^{\mathrm{s}} \subset \boldsymbol{V}^{\mathrm{s}}$, where the subscript $h>0$ indicates the level of spatial refinement. The associated family of meshes (for $h>0$ ) are supposed to be shape-regular and, for simplicity, quasi-uniform as well. Since the fluid velocity/pressure pair $\boldsymbol{V}_{h}^{\mathrm{f}} / Q_{h}$ fails to satisfy the inf-sup condition, we consider a symmetric pressure stabilization method defined by a non-negative bilinear form, $s_{h}: Q_{h} \times Q_{h} \rightarrow \mathbb{R}$ entering, for instance, the abstract framework of [9]. We define the semi-norm $\left|q_{h}\right|_{s_{h}} \stackrel{\text { def }}{=} \sqrt{s_{h}\left(q_{h}, q_{h}\right)}$ for all $q_{h} \in Q_{h}$.

Furthermore, we assume that the fluid and solid discretizations match at the interface, that is,

$$
\boldsymbol{\Lambda}_{\Sigma, h} \stackrel{\text { def }}{=}\left\{\left.\boldsymbol{v}_{h}^{\mathrm{f}}\right|_{\Sigma} / \boldsymbol{v}_{h}^{\mathrm{f}} \in \boldsymbol{V}_{h}^{\mathrm{f}}\right\}=\left\{\left.\boldsymbol{v}_{h}^{\mathrm{s}}\right|_{\Sigma} / \boldsymbol{v}_{h}^{\mathrm{s}} \in \boldsymbol{V}_{h}^{\mathrm{s}}\right\}
$$

and we set $\boldsymbol{V}_{\Sigma, h}^{\mathrm{f}} \stackrel{\text { def }}{=}\left\{\boldsymbol{v}_{h}^{\mathrm{f}} \in \boldsymbol{V}_{h}^{\mathrm{f}} /\left.\boldsymbol{v}_{h}^{\mathrm{f}}\right|_{\Sigma}=\mathbf{0}\right\}, \boldsymbol{V}_{\Sigma, h}^{\mathrm{s}} \stackrel{\text { def }}{=}\left\{\boldsymbol{v}_{h}^{\mathrm{s}} \in \boldsymbol{V}_{h}^{\mathrm{s}} /\left.\boldsymbol{v}_{h}^{\mathrm{s}}\right|_{\Sigma}=\mathbf{0}\right\}$ and

$$
\boldsymbol{W}_{h} \stackrel{\text { def }}{=}\left\{\left(\boldsymbol{v}_{h}^{\mathrm{f}}, \boldsymbol{v}_{h}^{\mathrm{s}}\right) \in \boldsymbol{V}_{h}^{\mathrm{f}} \times \boldsymbol{V}_{h}^{\mathrm{s}} /\left.\boldsymbol{v}_{h}^{\mathrm{f}}\right|_{\Sigma}=\left.\boldsymbol{v}_{h}^{\mathrm{s}}\right|_{\Sigma}\right\} \subset \boldsymbol{W} .
$$

We will consider the standard solid-sided and fluid-sided discrete lifting operators,

$$
\mathcal{L}_{h}^{\mathrm{s}}: \boldsymbol{\Lambda}_{\Sigma, h} \rightarrow \boldsymbol{V}_{h}^{\mathrm{s}}, \quad \mathcal{L}_{h}^{\mathrm{f}}: \boldsymbol{\Lambda}_{\Sigma, h} \rightarrow \boldsymbol{V}_{h}^{\mathrm{f}},
$$

defined for all $\boldsymbol{\xi}_{h} \in \boldsymbol{\Lambda}_{\Sigma, h}$, such that the nodal values of $\mathcal{L}_{h}^{\mathrm{s}} \boldsymbol{\xi}_{h}, \mathcal{L}_{h}^{\mathrm{f}} \boldsymbol{\xi}_{h}$ vanish out of $\Sigma$ and that $\left.\left(\mathcal{L}_{h}^{\mathrm{s}} \boldsymbol{\xi}_{h}\right)\right|_{\Sigma}=\left.\left(\mathcal{L}_{h}^{\mathrm{f}} \boldsymbol{\xi}_{h}\right)\right|_{\Sigma}=\boldsymbol{\xi}_{h}$.

In what follows, the symbol $(\cdot, \cdot)_{*}$ designates either the standard inner-product $(\cdot, \cdot)_{\Omega^{\mathrm{s}}}$ in $L^{2}\left(\Omega^{\mathrm{s}}\right)$, or its lumped-mass approximation $(\cdot, \cdot)_{\Omega^{\mathrm{s}}, h}$ (see, e.g., [28, Section 2.3] and [33, Chapter 15]). We also set

$$
a_{*}^{\mathrm{v}}\left(\dot{\boldsymbol{d}}_{h}, \boldsymbol{v}_{h}^{\mathrm{s}}\right) \stackrel{\text { def }}{=} \alpha_{0} \rho^{\mathrm{s}}\left(\dot{\boldsymbol{d}}_{h}, \boldsymbol{v}_{h}^{\mathrm{s}}\right)_{*}+\alpha_{1} a^{\mathrm{e}}\left(\dot{\boldsymbol{d}}_{h}, \boldsymbol{v}_{h}^{\mathrm{s}}\right)
$$

for all $\boldsymbol{d}_{h}, \boldsymbol{v}_{h}^{\mathrm{s}} \in \boldsymbol{V}_{h}^{\mathrm{s}}$. We denote by $\|\cdot\|_{\mathrm{v}, *}$ and $\|\cdot\|_{*}$ the norms associated to the inner-products $a_{*}^{\mathrm{v}}$ and $(\cdot, \cdot)_{*}$, respectively.

Remark 3 We recall that the norms $\|\cdot\|_{0, \Omega^{\mathrm{s}}}$ and $\|\cdot\|_{*}$ are equivalent in $\boldsymbol{V}_{h}^{\mathrm{s}}$, uniformly in $h$ (see, e.g., [33, Chapter 15]) and the same holds for $\|\cdot\|_{\mathrm{v}}$ and $\|\cdot\|_{\mathrm{v}, *}$. We will use the symbol $\lesssim$ to indicate an inequality written up to a multiplicative constant (independent of the physical and discretization parameters).

We consider the discrete reconstructions $\boldsymbol{L}_{h}^{\mathrm{e}}: \boldsymbol{V}^{\mathrm{s}} \rightarrow \boldsymbol{V}_{h}^{\mathrm{s}}$ and $\boldsymbol{L}_{h}^{\mathrm{v}}: \boldsymbol{V}_{h}^{\mathrm{s}} \rightarrow \boldsymbol{V}_{h}^{\mathrm{s}}$, of the elastic and viscous solid operators, defined through the relations:

$$
\left(\boldsymbol{L}_{h}^{\mathrm{e}} \boldsymbol{d}, \boldsymbol{v}_{h}^{\mathrm{s}}\right)_{*}=a^{\mathrm{e}}\left(\boldsymbol{d}, \boldsymbol{v}_{h}^{\mathrm{s}}\right), \quad\left(\boldsymbol{L}_{h}^{\mathrm{v}} \dot{\boldsymbol{d}}_{h}, \boldsymbol{v}_{h}^{\mathrm{s}}\right)_{*}=a_{*}^{\mathrm{v}}\left(\dot{\boldsymbol{d}}_{h}, \boldsymbol{v}_{h}^{\mathrm{s}}\right),
$$

for all $\left(\boldsymbol{d}, \dot{\boldsymbol{d}}_{h}, \boldsymbol{v}_{h}^{\mathrm{s}}\right) \in \boldsymbol{V}^{\mathrm{s}} \times \boldsymbol{V}_{h}^{\mathrm{s}} \times \boldsymbol{V}_{h}^{\mathrm{s}}$. We will also make use of the interface operator $\boldsymbol{B}_{h}: \boldsymbol{\Lambda}_{\Sigma, h} \rightarrow$ $\boldsymbol{\Lambda}_{\Sigma, h}$, defined by $\boldsymbol{B}_{h} \stackrel{\text { def }}{=}\left(\mathcal{L}_{h}^{\mathrm{s}}\right)^{\prime} \mathcal{L}_{h}^{\mathrm{s}}$, where $\left(\mathcal{L}_{h}^{\mathrm{s}}\right)^{\prime}$ stands for the adjoint operator of $\mathcal{L}_{h}^{\mathrm{s}}$ with respect to the lumped-mass inner product $(\cdot, \cdot)_{\Omega^{\mathrm{s}}, h}$ in $\boldsymbol{V}_{h}^{\mathrm{s}}$. Note that the interface operator $\boldsymbol{B}_{h}$ is selfadjoint, positive, definite and diagonal with respect to the finite element basis of $\boldsymbol{\Lambda}_{\Sigma, h}$. In order to simplify the presentation, we use the notation $\boldsymbol{B}_{h} \boldsymbol{v}_{h}^{\mathrm{s}}$ instead of $\boldsymbol{B}_{h}\left(\left.\boldsymbol{v}_{h}^{\mathrm{s}}\right|_{\Sigma}\right)$ for $\boldsymbol{v}_{h}^{\mathrm{s}} \in \boldsymbol{V}_{h}^{\mathrm{s}}$.

The space semi-discrete formulation of problem (5), including a possible mass-lumping approximation in the structure, reads therefore as follows: for all $t \in(0, T)$, find $\left(\boldsymbol{u}_{h}(t), \dot{\boldsymbol{d}}_{h}(t)\right) \in$ 
$\boldsymbol{W}_{h}, p_{h}(t) \in Q_{h}$ and $\boldsymbol{d}_{h}(t) \in \boldsymbol{V}_{h}^{\mathrm{s}}$ such that

$$
\left\{\begin{array}{l}
\dot{\boldsymbol{d}}_{h}=\partial_{t} \boldsymbol{d}_{h}, \\
\rho^{\mathrm{f}}\left(\partial_{t} \boldsymbol{u}_{h}, \boldsymbol{v}_{h}^{\mathrm{f}}\right)_{\Omega^{\mathrm{f}}}+a\left(\boldsymbol{u}_{h}, \boldsymbol{v}_{h}^{\mathrm{f}}\right)+b\left(p_{h}, \boldsymbol{v}_{h}^{\mathrm{f}}\right)-b\left(q_{h}, \boldsymbol{u}_{h}\right)+s_{h}\left(p_{h}, q_{h}\right) \\
\quad+\rho^{\mathrm{s}}\left(\partial_{t} \dot{\boldsymbol{d}}_{h}, \boldsymbol{v}_{h}^{\mathrm{s}}\right)_{*}+a^{\mathrm{e}}\left(\boldsymbol{d}_{h}, \boldsymbol{v}_{h}^{\mathrm{s}}\right)+a_{*}^{\mathrm{v}}\left(\dot{\boldsymbol{d}}_{h}, \boldsymbol{v}_{h}^{\mathrm{s}}\right)=l\left(\boldsymbol{v}_{h}^{\mathrm{f}}\right)
\end{array}\right.
$$

for all $\left(\boldsymbol{v}_{h}^{\mathrm{f}}, \boldsymbol{v}_{h}^{\mathrm{s}}\right) \in \boldsymbol{W}_{h}$ and $q_{h} \in Q_{h}$.

\subsection{Time discretization: coupling schemes}

This section is devoted to the discretization in time of (9). In the succeeding text, the parameter $\tau>0$ stands for the time-step length, $t_{n} \stackrel{\text { def }}{=} n \tau<T$, with $n \in \mathbb{N}$, and $\partial_{\tau} x^{n} \stackrel{\text { def }}{=}\left(x^{n}-x^{n-1}\right) / \tau$ for the first-order backward difference. The symbol $x^{n, \star}$ denotes the $r$-th order explicit extrapolation at time $t_{n}$, namely,

$$
x^{n, \star} \stackrel{\text { def }}{=}\left\{\begin{array}{lll}
0 & \text { if } & r=0, \\
x^{n-1} & \text { if } & r=1, \\
2 x^{n-1}-x^{n-2} & \text { if } & r=2 .
\end{array}\right.
$$

Basically, the explicit coupling schemes introduced in [21] build on a certain interface Robin consistency that can be recovered from the space semi-discrete formulation (9) with a masslumping approximation in the structure. In the spirit of [19], we propose an alternative derivation of these methods based on operator splitting (Sections 3.2.1-3.2.2). The benefit of this approach is that mass-lumping becomes optional and fully highlights its role on the explicit nature of the coupling schemes.

\subsubsection{Semi-implicit coupling schemes}

We first consider the following fractional-step time-marching of (9): for $n \geq 1$,

1. Find $\left(\boldsymbol{u}_{h}^{n}, \dot{\boldsymbol{d}}_{h}^{n-\frac{1}{2}}\right) \in \boldsymbol{W}_{h}$ and $p_{h}^{n} \in Q_{h}$ such that

$$
\left\{\begin{array}{l}
\rho^{\mathrm{f}}\left(\partial_{\tau} \boldsymbol{u}_{h}^{n}, \boldsymbol{v}_{h}^{\mathrm{f}}\right)_{\Omega^{\mathrm{f}}}+a\left(\boldsymbol{u}_{h}^{n}, \boldsymbol{v}_{h}^{\mathrm{f}}\right)+b\left(p_{h}^{n}, \boldsymbol{v}_{h}^{\mathrm{f}}\right)-b\left(q_{h}, \boldsymbol{u}_{h}^{n}\right)+s_{h}\left(p_{h}^{n}, q_{h}\right) \\
\quad+\frac{\rho^{\mathrm{s}}}{\tau}\left(\dot{\boldsymbol{d}}_{h}^{n-\frac{1}{2}}, \boldsymbol{v}_{h}^{\mathrm{s}}\right)_{*}=l\left(\boldsymbol{v}_{h}^{\mathrm{f}}\right)+\frac{\rho^{\mathrm{s}}}{\tau}\left(\dot{\boldsymbol{d}}_{h}^{n-1}, \boldsymbol{v}_{h}^{\mathrm{s}}\right)_{*}-a^{\mathrm{e}}\left(\boldsymbol{d}_{h}^{n, \star}, \boldsymbol{v}_{h}^{\mathrm{s}}\right)-a_{*}^{\mathrm{v}}\left(\dot{\boldsymbol{d}}_{h}^{n, \star}, \boldsymbol{v}_{h}^{\mathrm{s}}\right)
\end{array}\right.
$$

for all $\left(\boldsymbol{v}_{h}^{\mathrm{f}}, \boldsymbol{v}_{h}^{\mathrm{s}}\right) \in \boldsymbol{W}_{h}$ and for all $q_{h} \in Q_{h}$;

2. Find $\left(\boldsymbol{d}_{h}^{n}, \dot{\boldsymbol{d}}_{h}^{n}\right) \in \boldsymbol{V}_{h}^{\mathrm{s}} \times \boldsymbol{V}_{h}^{\mathrm{s}}$ such that

$$
\left\{\begin{array}{l}
\dot{\boldsymbol{d}}_{h}^{n}=\partial_{\tau} \boldsymbol{d}_{h}^{n} \\
\frac{\rho^{\mathrm{s}}}{\tau}\left(\dot{\boldsymbol{d}}_{h}^{n}-\dot{\boldsymbol{d}}_{h}^{n-\frac{1}{2}}, \boldsymbol{v}_{h}^{\mathrm{s}}\right)_{*}+a^{\mathrm{e}}\left(\boldsymbol{d}_{h}^{n}-\boldsymbol{d}_{h}^{n, \star}, \boldsymbol{v}_{h}^{\mathrm{s}}\right)+a_{*}^{\mathrm{v}}\left(\dot{\boldsymbol{d}}_{h}^{n}-\dot{\boldsymbol{d}}_{h}^{n, \star}, \boldsymbol{v}_{h}^{\mathrm{s}}\right)=0
\end{array}\right.
$$

for all $\boldsymbol{v}_{h}^{\mathrm{s}} \in \boldsymbol{V}_{h}^{\mathrm{s}}$.

Note that step (10) introduces and additional unknown, the intermediate solid velocity $\dot{\boldsymbol{d}}_{h}^{n-\frac{1}{2}}$, which is implicitly coupled to the fluid through the solid inertial term. In other words, step (10) implicitly treats the solid inertia within the fluid. The remaining solid viscoelastic contributions are treated explicitly in (10) via extrapolation. The end-of-step solid velocity $\dot{\boldsymbol{d}}_{h}^{n}$ is obtained 


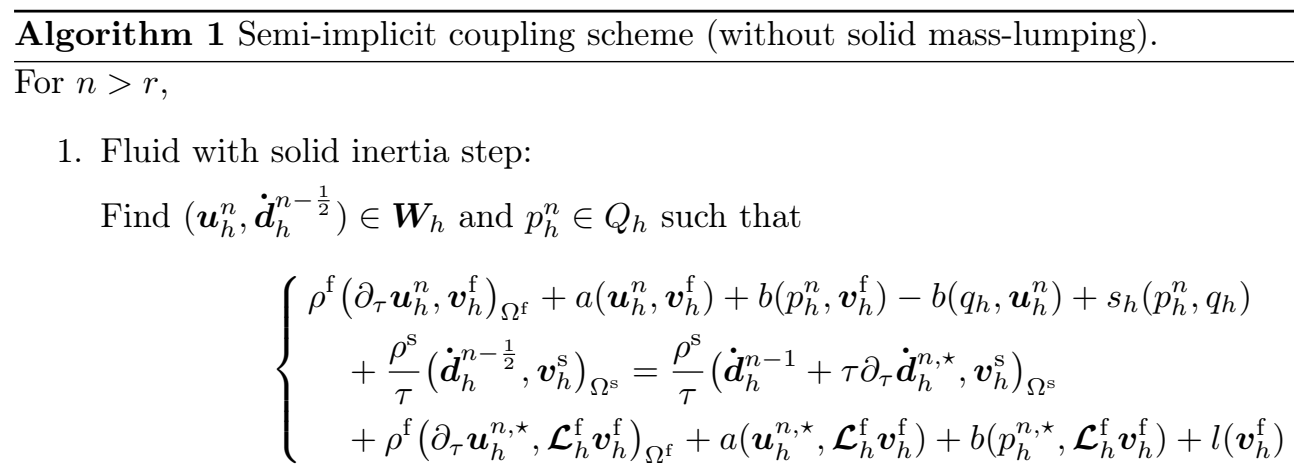

for all $\left(\boldsymbol{v}_{h}^{\mathrm{f}}, \boldsymbol{v}_{h}^{\mathrm{s}}\right) \in \boldsymbol{W}_{h}$ and $q_{h} \in Q_{h}$.

2. Solid step (Neumann):

Find $\left(\dot{\boldsymbol{d}}_{h}^{n}, \boldsymbol{d}_{h}^{n}\right) \in \boldsymbol{V}_{h}^{\mathrm{s}} \times \boldsymbol{V}_{h}^{\mathrm{s}}$ such that

$$
\left\{\begin{array}{l}
\dot{\boldsymbol{d}}_{h}^{n}=\partial_{\tau} \boldsymbol{d}_{h}^{n}, \\
\rho^{\mathrm{s}}\left(\partial_{\tau} \dot{\boldsymbol{d}}_{h}^{n}, \boldsymbol{v}_{h}^{\mathrm{s}}\right)_{\Omega^{\mathrm{s}}}+a^{\mathrm{e}}\left(\boldsymbol{d}_{h}^{n}, \boldsymbol{v}_{h}^{\mathrm{s}}\right)+a^{\mathrm{v}}\left(\dot{\boldsymbol{d}}_{h}^{n}, \boldsymbol{v}_{h}^{\mathrm{s}}\right) \\
\quad=-\rho^{\mathrm{f}}\left(\partial_{\tau} \boldsymbol{u}_{h}^{n}, \mathcal{L}_{h}^{\mathrm{f}} \boldsymbol{v}_{h}^{\mathrm{s}}\right)_{\Omega^{\mathrm{f}}}-a\left(\boldsymbol{u}_{h}^{n}, \mathcal{L}_{h}^{\mathrm{f}} \boldsymbol{v}_{h}^{\mathrm{s}}\right)-b\left(p_{h}^{n}, \mathcal{L}_{h}^{\mathrm{f}} \boldsymbol{v}_{h}^{\mathrm{s}}\right)
\end{array}\right.
$$

for all $\boldsymbol{v}_{h}^{\mathrm{s}} \in \boldsymbol{V}_{h}^{\mathrm{s}}$.

by solving the solid correction step (11). The implicit treatment of the solid inertia in (10) guarantees stability, while the extrapolation of the solid viscoelastic terms introduces a certain degree of splitting between the computation of the fluid and of the solid.

The correction step (11) can be reformulated as a standard solid (Neumann) problem by eliminating the quantitities $\left(\dot{\boldsymbol{d}}_{h}^{n-\frac{1}{2}}, \boldsymbol{d}_{h}^{n, \star}, \dot{\boldsymbol{d}}_{h}^{n, \star}\right)$. Indeed, by taking $\boldsymbol{v}_{h}^{\mathrm{f}}=\mathcal{L}_{h}^{\mathrm{f}} \boldsymbol{v}_{h}^{\mathrm{s}}$ and $q_{h}=0$ in $(10)$ and by adding the resulting expression to $(11)_{2}$, we get

$$
\rho^{\mathrm{s}}\left(\partial_{\tau} \dot{\boldsymbol{d}}_{h}^{n}, \boldsymbol{v}_{h}^{\mathrm{s}}\right)_{*}+a^{\mathrm{e}}\left(\boldsymbol{d}_{h}^{n}, \boldsymbol{v}_{h}^{\mathrm{s}}\right)+a_{*}^{\mathrm{v}}\left(\dot{\boldsymbol{d}}_{h}^{n}, \boldsymbol{v}_{h}^{\mathrm{s}}\right)=-\rho^{\mathrm{f}}\left(\partial_{\tau} \boldsymbol{u}_{h}^{n}, \mathcal{L}_{h}^{\mathrm{f}} \boldsymbol{v}_{h}^{\mathrm{s}}\right)_{\Omega^{\mathrm{f}}}-a\left(\boldsymbol{u}_{h}^{n}, \mathcal{L}_{h}^{\mathrm{f}} \boldsymbol{v}_{h}^{\mathrm{s}}\right)-b\left(p_{h}^{n}, \mathcal{L}_{h}^{\mathrm{f}} \boldsymbol{v}_{h}^{\mathrm{s}}\right)
$$

for all $\boldsymbol{v}_{h}^{\mathrm{s}} \in \boldsymbol{V}_{h}^{\mathrm{s}}$. Furthermore, for $n>r$, the extrapolated solid viscoelastic contribution in (10) can be eliminated owing to (14) at the time-steps $n-1, \ldots, n-r$. Since $\left.\boldsymbol{v}_{h}^{\mathrm{s}}\right|_{\Sigma}=\left.\boldsymbol{v}_{h}^{\mathrm{f}}\right|_{\Sigma}$ in (14), this yields

$-a^{\mathrm{e}}\left(\boldsymbol{d}_{h}^{n, \star}, \boldsymbol{v}_{h}^{\mathrm{s}}\right)-a_{*}^{\mathrm{v}}\left(\dot{\boldsymbol{d}}_{h}^{n, \star}, \boldsymbol{v}_{h}^{\mathrm{s}}\right)=\rho^{\mathrm{s}}\left(\partial_{\tau} \dot{\boldsymbol{d}}_{h}^{n, \star}, \boldsymbol{v}_{h}^{\mathrm{s}}\right)_{*}+\rho^{\mathrm{f}}\left(\partial_{\tau} \boldsymbol{u}_{h}^{n, \star}, \mathcal{L}_{h}^{\mathrm{f}} \boldsymbol{v}_{h}^{\mathrm{f}}\right)_{\Omega^{\mathrm{f}}}+a\left(\boldsymbol{u}_{h}^{n, \star}, \mathcal{L}_{h}^{\mathrm{f}} \boldsymbol{v}_{h}^{\mathrm{f}}\right)+b\left(p_{h}^{n, \star}, \mathcal{L}_{h}^{\mathrm{f}} \boldsymbol{v}_{h}^{\mathrm{f}}\right)$ for all $\left(\boldsymbol{v}_{h}^{\mathrm{f}}, \boldsymbol{v}_{h}^{\mathrm{s}}\right) \in \boldsymbol{W}_{h}$.

Algorithm 1 presents the resulting time-marching procedure without the mass-lumping approximation in the solid. It should be noted that this coupling scheme is not explicit, it is semi-implicit, since the fluid sub-problem has the same size as the original coupled problem (9).

\subsubsection{Generalized Robin-Neumann explicit coupling schemes}

If solid mass-lumping is considered in Algorithm 1, the contributions of the solid intermediate velocity $\dot{\boldsymbol{d}}_{h}^{n-\frac{1}{2}}$ can be rewritten exclusively in terms of the interface fluid velocity $\left.\boldsymbol{u}_{h}^{n}\right|_{\Sigma}$. Indeed, 
from [21, Lemma 1] and since $\left.\boldsymbol{u}_{h}^{n}\right|_{\Sigma}=\left.\dot{\boldsymbol{d}}_{h}^{n-\frac{1}{2}}\right|_{\Sigma}$, it follows that

$$
\left(\dot{\boldsymbol{d}}_{h}^{n-\frac{1}{2}}, \mathcal{L}_{h}^{\mathrm{s}} \boldsymbol{v}_{h}^{\mathrm{s}}\right)_{\Omega^{\mathrm{s}}, h}=\left(\boldsymbol{B}_{h} \dot{\boldsymbol{d}}_{h}^{n-\frac{1}{2}}, \boldsymbol{v}_{h}^{\mathrm{s}}\right)_{\Sigma}=\left(\boldsymbol{B}_{h} \boldsymbol{u}_{h}^{n}, \boldsymbol{v}_{h}^{\mathrm{f}}\right)_{\Sigma}
$$

for all $\left(\boldsymbol{v}_{h}^{\mathrm{f}}, \boldsymbol{v}_{h}^{\mathrm{s}}\right) \in \boldsymbol{W}_{h}$. In other words, after solid mass-lumping, the intermediate solid velocity $\dot{\boldsymbol{d}}_{h}^{n-\frac{1}{2}}$ disappears from the computation and, therefore, the coupling scheme becomes explicit. The resulting solution procedure is recalled in Algorithm 2.

Algorithm 2 Generalized Robin-Neumann explicit coupling schemes (from [19, 21]).

For $n>r$,

1. Fluid step (generalized Robin): find $\left(\boldsymbol{u}_{h}^{n}, p_{h}^{n}\right) \in \boldsymbol{V}_{h}^{\mathrm{f}} \times Q_{h}$ such that

$$
\left\{\begin{aligned}
\rho^{\mathrm{f}} & \left(\partial_{\tau} \boldsymbol{u}_{h}^{n}, \boldsymbol{v}_{h}^{\mathrm{f}}\right)_{\Omega^{\mathrm{f}}}+a\left(\boldsymbol{u}_{h}^{n}, \boldsymbol{v}_{h}^{\mathrm{f}}\right)+b\left(p_{h}^{n}, \boldsymbol{v}_{h}^{\mathrm{f}}\right)-b\left(q_{h}, \boldsymbol{u}_{h}^{n}\right)+s_{h}\left(p_{h}^{n}, q_{h}\right) \\
& +\frac{\rho^{\mathrm{s}}}{\tau}\left(\boldsymbol{B}_{h} \boldsymbol{u}_{h}^{n}, \boldsymbol{v}_{h}^{\mathrm{f}}\right)_{\Sigma}=\frac{\rho^{\mathrm{s}}}{\tau}\left(\boldsymbol{B}_{h}\left(\dot{\boldsymbol{d}}_{h}^{n-1}+\tau \partial_{\tau} \dot{\boldsymbol{d}}_{h}^{n, \star}\right), \boldsymbol{v}_{h}^{\mathrm{f}}\right)_{\Sigma} \\
& +\rho^{\mathrm{f}}\left(\partial_{\tau} \boldsymbol{u}_{h}^{n, \star}, \mathcal{L}_{h}^{\mathrm{f}} \boldsymbol{v}_{h}^{\mathrm{f}}\right)_{\Omega^{\mathrm{f}}}+a\left(\boldsymbol{u}_{h}^{n, \star}, \mathcal{L}_{h}^{\mathrm{f}} \boldsymbol{v}_{h}^{\mathrm{f}}\right)+b\left(p_{h}^{n, \star}, \mathcal{L}_{h}^{\mathrm{f}} \boldsymbol{v}_{h}^{\mathrm{f}}\right)+l\left(\boldsymbol{v}_{h}^{\mathrm{f}}\right)
\end{aligned}\right.
$$

for all $\left(\boldsymbol{v}_{h}^{\mathrm{f}}, q_{h}\right) \in \boldsymbol{V}_{h}^{\mathrm{f}} \times Q_{h}$.

2. Solid step (Neumann): find $\left(\dot{\boldsymbol{d}}_{h}^{n}, \boldsymbol{d}_{h}^{n}\right) \in \boldsymbol{V}_{h}^{\mathrm{s}} \times \boldsymbol{V}_{h}^{\mathrm{s}}$ such that

$$
\left\{\begin{array}{l}
\dot{\boldsymbol{d}}_{h}^{n}=\partial_{\tau} \boldsymbol{d}_{h}^{n}, \\
\rho^{\mathrm{s}}\left(\partial_{\tau} \dot{\boldsymbol{d}}_{h}^{n}, \boldsymbol{v}_{h}^{\mathrm{s}}\right)_{\Omega^{\mathrm{s}}, h}+a^{\mathrm{e}}\left(\boldsymbol{d}_{h}^{n}, \boldsymbol{v}_{h}^{\mathrm{s}}\right)+a_{h}^{\mathrm{v}}\left(\dot{\boldsymbol{d}}_{h}^{n}, \boldsymbol{v}_{h}^{\mathrm{s}}\right) \\
\quad=-\rho^{\mathrm{f}}\left(\partial_{\tau} \boldsymbol{u}_{h}^{n}, \mathcal{L}_{h}^{\mathrm{f}} \boldsymbol{v}_{h}^{\mathrm{s}}\right)_{\Omega^{\mathrm{f}}}-a\left(\boldsymbol{u}_{h}^{n}, \mathcal{L}_{h}^{\mathrm{f}} \boldsymbol{v}_{h}^{\mathrm{s}}\right)-b\left(p_{h}^{n}, \mathcal{L}_{h}^{\mathrm{f}} \boldsymbol{v}_{h}^{\mathrm{s}}\right)
\end{array}\right.
$$

for all $\boldsymbol{v}_{h}^{\mathrm{s}} \in \boldsymbol{V}_{h}^{\mathrm{s}}$.

Instead of the usual interface identity operator involved in standard Robin conditions for the fluid (see, e.g., $[2,20]$ ), the fluid sub-problem (15) incorporates the operator $\boldsymbol{B}_{h}$ which consistently accounts for the inertial effects of the solid within the fluid (see [21]). This explains the terminology generalized Robin. Note that these generalized Robin conditions must be distinguished from those considered in [22, 31, 24, 8, 30], which involve solid surface differential operators.

Remark 4 Algorithms 1 and 2 are essentially the same coupling scheme: they only differ on the discrete treatment of the inner-product $(\cdot, \cdot)_{\Omega^{\mathrm{s}}}$.

Remark 5 Algorithms 1 and 2 are multi-step methods for $r>0$, meaning that an initial condition $\left(\boldsymbol{u}_{h}^{0}, \boldsymbol{d}_{h}^{0}, \dot{\boldsymbol{d}}_{h}^{0}\right)$ is not enough to initiate the time marching. In the case $r=1$, we propose to obtain $\left(\boldsymbol{u}_{h}^{1}, \boldsymbol{d}_{h}^{1}, \dot{\boldsymbol{d}}_{h}^{1}\right)$ with one step of the scheme with $r=0$. Similarly, the scheme with $r=2$ is initialized by performing one step of the scheme with $r=1$ and so on.

For further reference in the convergence analysis of Section 4, we now recall the energy stability result established in [21, Thereom 4] for Algorithm 2. We will show that it also holds for Algorithm 1.

We begin with the following fundamental property of Algorithms 1 and 2: they are nothing but interface kinematic perturbations of a fully implicit time-discretization of (9) (implicit coupling scheme).

$\operatorname{RR} n^{\circ} 8670$ 
Lemma 1 Let $\left\{\left(\boldsymbol{u}_{h}^{n}, p_{h}^{n}, \boldsymbol{d}_{h}^{n}, \dot{\boldsymbol{d}}_{h}^{n}\right)\right\}_{n>r}$ be the sequence given by Algorithm 1 or 2. For $n>r$, there holds

$$
\boldsymbol{u}_{h}^{n}=\dot{\boldsymbol{d}}_{h}^{n}+\frac{\tau}{\rho^{\mathrm{s}}}\left[\boldsymbol{L}_{h}^{\mathrm{e}}\left(\boldsymbol{d}_{h}^{n}-\boldsymbol{d}_{h}^{n, \star}\right)+\boldsymbol{L}_{h}^{\mathrm{v}}\left(\dot{\boldsymbol{d}}_{h}^{n}-\dot{\boldsymbol{d}}_{h}^{n, \star}\right)\right] \quad \text { on } \quad \Sigma
$$

and

$$
\left\{\begin{array}{l}
\dot{\boldsymbol{d}}_{h}^{n}=\partial_{\tau} \boldsymbol{d}_{h}^{n}, \\
\rho^{\mathrm{f}}\left(\partial_{\tau} \boldsymbol{u}_{h}^{n}, \boldsymbol{v}_{h}^{\mathrm{f}}\right)_{\Omega^{\mathrm{f}}}+a\left(\boldsymbol{u}_{h}^{n}, \boldsymbol{v}_{h}^{\mathrm{f}}\right)+b\left(p_{h}^{n}, \boldsymbol{v}_{h}^{\mathrm{f}}\right)-b\left(q_{h}, \boldsymbol{u}_{h}^{n}\right)+s_{h}\left(p_{h}^{n}, q_{h}\right) \\
\quad+\rho^{\mathrm{s}}\left(\partial_{\tau} \dot{\boldsymbol{d}}_{h}^{n}, \boldsymbol{v}_{h}^{\mathrm{s}}\right)_{*}+a^{\mathrm{e}}\left(\boldsymbol{d}_{h}^{n}, \boldsymbol{v}_{h}^{\mathrm{s}}\right)+a_{*}^{\mathrm{v}}\left(\dot{\boldsymbol{d}}_{h}^{n}, \boldsymbol{v}_{h}^{\mathrm{s}}\right)=l\left(\boldsymbol{v}_{h}^{\mathrm{f}}\right)
\end{array}\right.
$$

for all $\left(\boldsymbol{v}_{h}^{\mathrm{f}}, \boldsymbol{v}_{h}^{\mathrm{s}}\right) \in \boldsymbol{W}_{h}$ and $q_{h} \in Q_{h}$.

Proof 1 The result for Algorithm 2 has been established in [21, Lemma 2]. We now detail the proof for Algorithm 1. First, the substitution of the superscript ${ }^{n}$ by ${ }^{n, \star}$ in (13), which is valid for $n>r$, gives

$$
\rho^{\mathrm{s}}\left(\partial_{\tau} \dot{\boldsymbol{d}}_{h}^{n, \star}, \boldsymbol{v}_{h}^{\mathrm{s}}\right)_{\Omega^{\mathrm{s}}}+a^{\mathrm{e}}\left(\boldsymbol{d}_{h}^{n, \star}, \boldsymbol{v}_{h}^{\mathrm{s}}\right)+a^{\mathrm{v}}\left(\dot{\boldsymbol{d}}_{h}^{n, \star}, \boldsymbol{v}_{h}^{\mathrm{s}}\right)=-\rho^{\mathrm{f}}\left(\partial_{\tau} \boldsymbol{u}_{h}^{n, \star}, \mathcal{L}_{h}^{\mathrm{f}} \boldsymbol{v}_{h}^{\mathrm{s}}\right)_{\Omega^{\mathrm{f}}}-a\left(\boldsymbol{u}_{h}^{n, \star}, \mathcal{L}_{h}^{\mathrm{f}} \boldsymbol{v}_{h}^{\mathrm{s}}\right)-b\left(p_{h}^{n, \star}, \mathcal{L}_{h}^{\mathrm{f}} \boldsymbol{v}_{h}^{\mathrm{s}}\right)
$$

for all $\boldsymbol{v}_{h}^{\mathrm{s}} \in \boldsymbol{V}_{h}^{\mathrm{s}}$. Then, the choice $\boldsymbol{v}_{h}^{\mathrm{f}}=\mathcal{L}_{h}^{\mathrm{f}} \boldsymbol{v}_{h}^{\mathrm{s}}$ and $q_{h}=0$ in (13) yields

$$
\begin{aligned}
& \rho^{\mathrm{f}}\left(\partial_{\tau} \boldsymbol{u}_{h}^{n}, \mathcal{L}_{h}^{\mathrm{f}} \boldsymbol{v}_{h}^{\mathrm{s}}\right)_{\Omega^{\mathrm{f}}}+a\left(\boldsymbol{u}_{h}^{n}, \mathcal{L}_{h}^{\mathrm{f}} \boldsymbol{v}_{h}^{\mathrm{s}}\right)+b\left(p_{h}^{n}, \mathcal{L}_{h}^{\mathrm{f}} \boldsymbol{v}_{h}^{\mathrm{s}}\right)+\frac{\rho^{\mathrm{s}}}{\tau}\left(\dot{\boldsymbol{d}}_{h}^{n-\frac{1}{2}}, \boldsymbol{v}_{h}^{\mathrm{s}}\right)_{\Omega^{\mathrm{s}}} \\
& \quad=\frac{\rho^{\mathrm{s}}}{\tau}\left(\dot{\boldsymbol{d}}_{h}^{n-1}+\tau \partial_{\tau} \dot{\boldsymbol{d}}_{h}^{n, \star}, \boldsymbol{v}_{h}^{\mathrm{s}}\right)_{\Omega^{\mathrm{s}}}+\rho^{\mathrm{f}}\left(\partial_{\tau} \boldsymbol{u}_{h}^{n, \star}, \mathcal{L}_{h}^{\mathrm{f}} \boldsymbol{v}_{h}^{\mathrm{s}}\right)_{\Omega^{\mathrm{f}}}+a\left(\boldsymbol{u}_{h}^{n, \star}, \mathcal{L}_{h}^{\mathrm{f}} \boldsymbol{v}_{h}^{\mathrm{s}}\right)+b\left(p_{h}^{n, \star}, \mathcal{L}_{h}^{\mathrm{f}} \boldsymbol{v}_{h}^{\mathrm{s}}\right)
\end{aligned}
$$

for all $\boldsymbol{v}_{h}^{\mathrm{s}} \in \boldsymbol{V}_{h}^{\mathrm{s}}$. Finally, by adding the relations (20), (19) and (13), we get

$$
\frac{\rho^{\mathrm{s}}}{\tau}\left(\dot{\boldsymbol{d}}_{h}^{n-\frac{1}{2}}-\dot{\boldsymbol{d}}_{h}^{n-1}, \boldsymbol{v}_{h}^{\mathrm{s}}\right)_{\Omega^{\mathrm{s}}}+a^{\mathrm{e}}\left(\boldsymbol{d}_{h}^{n}-\boldsymbol{d}_{h}^{n, \star}, \boldsymbol{v}_{h}^{\mathrm{s}}\right)+a^{\mathrm{v}}\left(\dot{\boldsymbol{d}}_{h}^{n}-\dot{\boldsymbol{d}}_{h}^{n, \star}, \boldsymbol{v}_{h}^{\mathrm{s}}\right)=0
$$

for all $\boldsymbol{v}_{h}^{\mathrm{s}} \in \boldsymbol{V}_{h}^{\mathrm{s}}$, or equivalently, owing to (8),

$$
\dot{\boldsymbol{d}}_{h}^{n-\frac{1}{2}}=\dot{\boldsymbol{d}}_{h}^{n}+\frac{\tau}{\rho^{\mathrm{s}}}\left[\boldsymbol{L}_{h}^{\mathrm{e}}\left(\boldsymbol{d}_{h}^{n}-\boldsymbol{d}_{h}^{n, \star}\right)+\boldsymbol{L}_{h}^{\mathrm{v}}\left(\dot{\boldsymbol{d}}_{h}^{n}-\dot{\boldsymbol{d}}_{h}^{n, \star}\right)\right] .
$$

The transmission condition (17) hence follows from (12), which enforces $\boldsymbol{u}_{h}^{n}=\dot{\boldsymbol{d}}_{h}^{n-\frac{1}{2}}$ on $\Sigma$. At last, the kinetic relation $(18)_{2}$ can be recovered from the summation of (12), (19) and (21).

The next theorem states the energy stability of Algorithms 1 and 2.

Theorem 2 Assume that $\boldsymbol{h}=\mathbf{0}$ (free system) and let

$$
\left\{\left(\boldsymbol{u}_{h}^{n}, p_{h}^{n}, \boldsymbol{d}_{h}^{n}, \dot{\boldsymbol{d}}_{h}^{n}\right)\right\}_{n>r} \subset \boldsymbol{V}_{h}^{\mathrm{f}} \times Q_{h} \times \boldsymbol{V}_{h}^{\mathrm{s}} \times \boldsymbol{V}_{h}^{\mathrm{s}}
$$

be the sequence given by Algorithm 1 or 2 with the initialization steps detailed in Remark 5. We denote by

and by

$$
E_{h}^{n} \stackrel{\text { def }}{=} \frac{\rho^{\mathrm{f}}}{2}\left\|\boldsymbol{u}_{h}^{n}\right\|_{0, \Omega^{\mathrm{f}}}^{2}+\frac{\rho^{\mathrm{s}}}{2}\left\|\dot{\boldsymbol{d}}_{h}^{n}\right\|_{0, \Omega^{\mathrm{s}}}^{2}+\frac{1}{2}\left\|\boldsymbol{d}_{h}^{n}\right\|_{\mathrm{e}}^{2}
$$

$D_{h}^{n} \stackrel{\text { def }}{=} \frac{\rho^{\mathrm{f}}}{\tau}\left\|\boldsymbol{u}_{h}^{n}-\boldsymbol{u}_{h}^{n-1}\right\|_{0, \Omega^{\mathrm{f}}}^{2}+\frac{\rho^{\mathrm{s}}}{\tau}\left\|\dot{\boldsymbol{d}}_{h}^{n}-\dot{\boldsymbol{d}}_{h}^{n-1}\right\|_{0, \Omega^{\mathrm{s}}}^{2}+\frac{1}{\tau}\left\|\boldsymbol{d}_{h}^{n}-\boldsymbol{d}_{h}^{n-1}\right\|_{\mathrm{e}}^{2}+2 \mu\left\|\boldsymbol{\epsilon}\left(\boldsymbol{u}_{h}^{n}\right)\right\|_{0, \Omega^{\mathrm{f}}}^{2}+\left|p_{h}^{n}\right|_{s_{h}}^{2}+\left\|\dot{\boldsymbol{d}}_{h}^{n}\right\|_{\mathrm{v}}^{2}$,

the total discrete energy and dissipation, respectively. The following a priori energy estimates hold: 
- $r=0$ or $r=1:$ for $n>r$

$$
E_{h}^{n}+\tau \sum_{m=r+1}^{n} D_{h}^{m} \lesssim E_{h}^{0}
$$

- $r=2$ : for $n \geq 3$

$$
E_{h}^{n}+\tau \sum_{m=3}^{n} D_{h}^{m} \lesssim \exp \left(\frac{t_{n} \gamma}{1-\gamma \tau}\right) E_{h}^{0}
$$

provided that the following set of conditions holds:

$$
\left\{\begin{aligned}
\tau\left(\alpha_{0}+\alpha_{1}\left(\frac{\omega_{\mathrm{e}}}{h}\right)^{2}\right) & <\delta \\
\tau^{5}\left(\frac{\omega_{\mathrm{e}}}{h}\right)^{6}+\tau^{2}\left(\frac{\omega_{\mathrm{e}}}{h}\right)^{2}\left(\alpha_{0}+\alpha_{1}\left(\frac{\omega_{\mathrm{e}}}{h}\right)^{2}\right) & <\gamma \\
\tau \gamma & <1
\end{aligned}\right.
$$

where $\omega_{\mathrm{e}} \stackrel{\text { def }}{=} C_{\text {inv }} \sqrt{\beta_{\mathrm{e}} / \rho^{\mathrm{s}}}, 0 \leq \delta \leq 1$ and $\gamma>0$.

Proof 2 For Algorithm 2 this result was established in [21, Thereom 4]. The result for Algorithm 1 follows from Lemma 1 by using the same arguments as in [21].

The above result shows that Algorithms 1 and 2 retain the stability properties of the original explicit Robin-Neumann schemes introduced in [20, 17], for the coupling with thin-walled structures. Extensive numerical evidence reported in [21, Section 6] demonstrates, however, that their accuracy is not preserved. More precisely, the numerically observed behavior of the energy error is $\mathcal{O}\left(\tau^{2^{r-1}} / h^{\frac{1}{2}}\right)$ for Algorithm 2, whereas an $\mathcal{O}\left(\tau^{2^{r-1}}\right)$ is obtained, both from the numerical and theoretical standpoint, with the Robin-Neumann schemes in [20, 17]. The purpose of the present paper is to identify the source of this accuracy loss, namely,

- Is solid mass-lumping the cause of the $h$ non-uniformity?

- Do Algorithms 1 and 2 deliver the same overall convergence rate?

All these fundamental questions will be answered in Section 4, via an a priori error analysis that covers both Algorithm 1 and 2. These theoretical findings will be then supported by further numerical evidence reported in Section 5.

\subsubsection{Alternative splitting scheme}

For further reference in the discussion and the numerical experiments of Section 5, we now recall the splitting scheme recently introduced in [7]. Applied to the coupled problem (5) this method yields the solution procedure reported in Algorithm 3, where $0 \leq \beta \leq 1$ denotes a free parameter. Note that this splitting treats implicitly the solid damping in (25). For a purely elastic structure (i.e., $\alpha_{0}=\alpha_{1}=0$ in (2)), Algorithm 3 with $\beta=0$ is the same as Algorithm 1 with $r=0$, since the relations (10)-(11) follow from (25)-(26).

More generally, the time-splittings provided by Algorithms 1 and 3 differ in the following two ingredients:

- the treatment of the solid damping;

- the consistency of the extrapolations on the interface.

$\mathrm{RR} \mathrm{n}^{\circ} 8670$ 
Algorithm 3 Splitting scheme from [7].

1. Fluid with solid inertia and damping step: find $\left(\boldsymbol{u}_{h}^{n}, \dot{\boldsymbol{d}}_{h}^{n-\frac{1}{2}}\right) \in \boldsymbol{W}_{h}$ and $p_{h}^{n} \in Q_{h}$ such that

$$
\left\{\begin{array}{l}
\rho^{\mathrm{f}}\left(\partial_{\tau} \boldsymbol{u}_{h}^{n}, \boldsymbol{v}_{h}^{\mathrm{f}}\right)_{\Omega^{\mathrm{f}}}+a\left(\boldsymbol{u}_{h}^{n}, \boldsymbol{v}_{h}^{\mathrm{f}}\right)+b\left(p_{h}^{n}, \boldsymbol{v}_{h}^{\mathrm{f}}\right)-b\left(q_{h}, \boldsymbol{u}_{h}^{n}\right)+s_{h}\left(p_{h}^{n}, q_{h}\right) \\
\quad+\frac{\rho^{\mathrm{s}}}{\tau}\left(\dot{\boldsymbol{d}}_{h}^{n-\frac{1}{2}}, \boldsymbol{v}_{h}^{\mathrm{s}}\right)_{\Omega^{\mathrm{s}}}+a^{\mathrm{v}}\left(\dot{\boldsymbol{d}}_{h}^{n-\frac{1}{2}}, \boldsymbol{v}_{h}^{\mathrm{s}}\right)=\frac{\rho^{\mathrm{s}}}{\tau}\left(\dot{\boldsymbol{d}}_{h}^{n-1}, \boldsymbol{v}_{h}^{\mathrm{s}}\right)_{\Omega^{\mathrm{s}}} \\
\quad-\beta\left(p_{h}^{n-1}, \boldsymbol{v}_{h}^{\mathrm{f}} \cdot \boldsymbol{n}^{\mathrm{f}}\right)_{\Sigma}+l\left(\boldsymbol{v}_{h}^{\mathrm{f}}\right)
\end{array}\right.
$$

for all $\left(\boldsymbol{v}_{h}^{\mathrm{f}}, \boldsymbol{v}_{h}^{\mathrm{s}}\right) \in \boldsymbol{W}_{h}$ and $q_{h} \in Q_{h}$.

2. Solid elastic step: find $\left(\dot{\boldsymbol{d}}_{h}^{n}, \boldsymbol{d}_{h}^{n}\right) \in \boldsymbol{V}_{h}^{\mathrm{s}} \times \boldsymbol{V}_{h}^{\mathrm{s}}$ such that

$$
\left\{\begin{array}{l}
\dot{\boldsymbol{d}}_{h}^{n}=\partial_{\tau} \boldsymbol{d}_{h}^{n} \\
\frac{\rho^{\mathrm{s}}}{\tau}\left(\dot{\boldsymbol{d}}_{h}^{n}-\dot{\boldsymbol{d}}_{h}^{n-\frac{1}{2}}, \boldsymbol{v}_{h}^{\mathrm{s}}\right)_{\Omega^{\mathrm{s}}}+a^{\mathrm{e}}\left(\boldsymbol{d}_{h}^{n}, \boldsymbol{v}_{h}^{\mathrm{s}}\right)=\beta\left(p_{h}^{n-1}, \boldsymbol{v}_{h}^{\mathrm{s}} \cdot \boldsymbol{n}^{\mathrm{f}}\right)_{\Sigma}
\end{array}\right.
$$

for all $\boldsymbol{v}_{h}^{\mathrm{s}} \in \boldsymbol{V}_{h}^{\mathrm{s}}$.

Algorithm 3 treats implicitly the whole solid hydrodynamic contributions (inertia and damping) in step (25), whereas in Algorithm 1 only the solid inertial effects are included in the left-hand side of (12). This induces two important drawbacks with respect to Algorithm 1. First, the solid mass-lumping approximation in Algorithm 3 does not make the coupling scheme explicit; and second, the solid elastic step (26) cannot be reformulated as the standard solid (Neumann) problem (16), since the intermediate velocity cannot be eliminated $\dot{\boldsymbol{d}}_{h}^{n-\frac{1}{2}}$. Indeed, by taking $\boldsymbol{v}_{h}^{\mathrm{f}}=\mathcal{L}_{h}^{\mathrm{f}} \boldsymbol{v}_{h}^{\mathrm{s}}$ and $q_{h}=0$ in $(25)$ and by adding the resulting expression to $(26)_{2}$, we get

$\rho^{\mathrm{s}}\left(\partial_{\tau} \dot{\boldsymbol{d}}_{h}^{n}, \boldsymbol{v}_{h}^{\mathrm{s}}\right)_{\Omega^{\mathrm{s}}}+a^{\mathrm{e}}\left(\boldsymbol{d}_{h}^{n}, \boldsymbol{v}_{h}^{\mathrm{s}}\right)=-a^{\mathrm{v}}\left(\dot{\boldsymbol{d}}_{h}^{n-\frac{1}{2}}, \boldsymbol{v}_{h}^{\mathrm{s}}\right)-\rho^{\mathrm{f}}\left(\partial_{\tau} \boldsymbol{u}_{h}^{n}, \mathcal{L}_{h}^{\mathrm{f}} \boldsymbol{v}_{h}^{\mathrm{s}}\right)_{\Omega^{\mathrm{f}}}-a\left(\boldsymbol{u}_{h}^{n}, \mathcal{L}_{h}^{\mathrm{f}} \boldsymbol{v}_{h}^{\mathrm{s}}\right)-b\left(p_{h}^{n}, \mathcal{L}_{h}^{\mathrm{f}} \boldsymbol{v}_{h}^{\mathrm{s}}\right)$

for all $\boldsymbol{v}_{h}^{\mathrm{s}} \in \boldsymbol{V}_{h}^{\mathrm{s}}$.

With regard to consistency, the interface term $\beta\left(p_{h}^{n-1}, \boldsymbol{v}_{h}^{\mathrm{f}} \cdot \boldsymbol{n}^{\mathrm{f}}\right)_{\Sigma}$ in Algorithm 3 is introduced, in a rather ad hoc fashion, with the aim of improving the accuracy of the time-splitting. Mathematically sound results on the stability and the consistency of Algoritm 3 for $\beta>0$ are not known. Numerical evidence suggests that the best accuracy is obtained with $\beta=1$ (see [7]). In Section 5 we will show that Algorithm 3 also suffers from the non-uniformity convergence issues discussed in Section 3.2.2.

\section{Convergence analysis}

This section is devoted to the convergence analysis of the coupling schemes given by Algorithms 1 and 2 .

\subsection{Preliminaries}

For all $\boldsymbol{d}_{h}, \boldsymbol{w}_{h} \in \boldsymbol{V}_{h}^{\mathrm{s}}$, the relation

$$
\delta_{h}\left(\boldsymbol{d}_{h}, \boldsymbol{w}_{h}\right) \stackrel{\text { def }}{=}\left(\boldsymbol{d}_{h}, \boldsymbol{w}_{h}\right)_{\Omega^{\mathrm{s}}}-\left(\boldsymbol{d}_{h}, \boldsymbol{w}_{h}\right)_{*}
$$


will denote the quadrature error. Without mass-lumping in the solid we have $\delta_{h}=0$, otherwise there holds

$$
\left|\delta_{h}\left(\boldsymbol{d}_{h}, \boldsymbol{w}_{h}\right)\right| \lesssim h\left\|\boldsymbol{d}_{h}\right\|_{1, \Omega^{\mathrm{s}}}\left\|\boldsymbol{w}_{h}\right\|_{0, \Omega^{\mathrm{s}}}
$$

for all $\boldsymbol{d}_{h}, \boldsymbol{w}_{h} \in \boldsymbol{V}_{h}^{\text {s }}$ (see, e.g., [33, Lemma 15.1]). Due to the particular form of the bilinear form $a^{\mathrm{e}}$, two positive constants $\alpha_{\mathrm{e}}, \beta_{\mathrm{e}}>0$ exist such that

$$
\alpha_{\mathrm{e}}\left\|\boldsymbol{d}_{h}\right\|_{1, \Omega^{\mathrm{s}}}^{2} \leq a^{\mathrm{e}}\left(\boldsymbol{d}_{h}, \boldsymbol{d}_{h}\right) \leq \beta_{\mathrm{e}}\left\|\boldsymbol{d}_{h}\right\|_{1, \Omega^{\mathrm{s}}}^{2}
$$

for all $\boldsymbol{d}_{h} \in \boldsymbol{V}_{h}^{\mathrm{s}}$. Thus, using an inverse inequality (see, e.g., [5, Chapter 4]), we have the following estimates:

$$
\begin{array}{rlrl}
\left\|\boldsymbol{v}_{h}^{\mathrm{s}}\right\|_{\mathrm{e}}^{2} & \leq \frac{\beta_{\mathrm{e}} C_{\mathrm{inv}}^{2}}{h^{2}}\left\|\boldsymbol{v}_{h}^{\mathrm{s}}\right\|_{0, \Omega^{\mathrm{s}}}^{2}, & \left\|\boldsymbol{v}_{h}^{\mathrm{s}}\right\|_{\mathrm{v}}^{2} & \leq\left(\alpha_{0} \rho^{\mathrm{s}}+\alpha_{1} \frac{\beta_{\mathrm{e}} C_{\mathrm{inv}}^{2}}{h^{2}}\right)\left\|\boldsymbol{v}_{h}^{\mathrm{s}}\right\|_{0, \Omega^{\mathrm{s}}}^{2}, \\
\left\|\boldsymbol{L}_{h}^{\mathrm{e}} \boldsymbol{v}_{h}^{\mathrm{s}}\right\|_{\mathrm{e}} \leq \frac{\beta_{\mathrm{e}} C_{\mathrm{inv}}^{2}}{h^{2}}\left\|\boldsymbol{v}_{h}^{\mathrm{s}}\right\|_{\mathrm{e}}, & \left\|\boldsymbol{L}_{h}^{\mathrm{v}} \boldsymbol{v}_{h}^{\mathrm{s}}\right\|_{\mathrm{v}} \leq\left(\alpha_{0} \rho^{\mathrm{s}}+\alpha_{1} \frac{\beta_{\mathrm{e}} C_{\mathrm{inv}}^{2}}{h^{2}}\right)\left\|\boldsymbol{v}_{h}^{\mathrm{s}}\right\|_{\mathrm{v}}, \\
\left\|\boldsymbol{L}_{h}^{\mathrm{e}} \boldsymbol{v}_{h}^{\mathrm{s}}\right\|_{0, \Omega^{\mathrm{s}}}^{2} \leq \frac{\beta_{\mathrm{e}} C_{\mathrm{inv}}^{2}}{h^{2}}\left\|\boldsymbol{v}_{h}^{\mathrm{s}}\right\|_{\mathrm{e}}^{2}, & \left\|\boldsymbol{L}_{h}^{\mathrm{v}} \boldsymbol{v}_{h}^{\mathrm{s}}\right\|_{0, \Omega^{\mathrm{s}}}^{2} \leq\left(\alpha_{0} \rho^{\mathrm{s}}+\alpha_{1} \frac{\beta_{\mathrm{e}} C_{\mathrm{inv}}^{2}}{h^{2}}\right)\left\|\boldsymbol{v}_{h}^{\mathrm{s}}\right\|_{\mathrm{v}}^{2},
\end{array}
$$

valid for all $\boldsymbol{v}_{h}^{\mathrm{s}} \in \boldsymbol{V}_{h}^{\mathrm{s}}$.

\subsection{Projection operators}

We introduce the Ritz-projector $\boldsymbol{\pi}_{h}^{\mathrm{e}}: \boldsymbol{V}^{\mathrm{s}} \rightarrow \boldsymbol{V}_{h}^{\mathrm{s}}$ defined, for each $\boldsymbol{d} \in \boldsymbol{V}^{\mathrm{s}}$, by the relation

$$
a^{\mathrm{e}}\left(\boldsymbol{d}-\boldsymbol{\pi}_{h}^{\mathrm{e}} \boldsymbol{d}, \boldsymbol{v}_{h}^{\mathrm{s}}\right)=0
$$

for all $\boldsymbol{v}_{h}^{\mathrm{s}} \in \boldsymbol{V}_{h}^{\mathrm{s}}$. Furthermore, we assume that the following error estimate holds

$$
\left\|\boldsymbol{d}-\boldsymbol{\pi}_{h}^{\mathrm{e}} \boldsymbol{d}\right\|_{\mathrm{e}} \lesssim h \beta_{\mathrm{e}}^{\frac{1}{2}}\|\boldsymbol{d}\|_{2, \Omega^{\mathrm{s}}}
$$

for all $\boldsymbol{d} \in \boldsymbol{V}^{\mathrm{s}} \cap\left[H^{2}\left(\Omega^{\mathrm{s}}\right)\right]^{d}$. We denote by $\Pi_{h}: Q \rightarrow Q_{h}$ the projection operator associated to the weak-consistency of the pressure stabilization operator $s_{h}$, for which we assume that

$$
\left\|p-\Pi_{h} p\right\|_{Q}+\left|\Pi_{h} p\right|_{s_{h}} \lesssim \frac{h}{\mu^{\frac{1}{2}}}\|p\|_{1, \Omega^{\mathrm{f}}} \quad \forall p \in Q \cap H^{1}\left(\Omega^{\mathrm{f}}\right) .
$$

Remark 6 An example of operator $s_{h}$ is given by (see, e.g., [6]):

$$
s_{h}\left(p_{h}, q_{h}\right)=\frac{\kappa h^{2}}{\mu}\left(\nabla p_{h}, \nabla q_{h}\right)_{\Omega^{\mathrm{f}}}
$$

for all $p_{h}, q_{h} \in Q_{h}$, where $\kappa>0$ is a user-defined parameter. In this case, the assumption (34) holds with $\Pi_{h}$ being the $L^{2}$-projection onto $Q_{h}$. This pressure stabilization method is considered in the numerical experiments of Section 5. Further examples are discussed in [9, Section 3.1.1].

The definition of the fluid and solid velocity projection operators, which will be assumed to match at the interface, is more delicate. This interface matching assumption guarantees that Lemma 4 (i.e., the discrete error counterpart of (17)) holds. This point is fundamental for the error estimates of Section 4.4. The velocity projection operators are defined by distinguishing the cases $\alpha_{1}=0$ and $\alpha_{1}>0$ (see Remark 7).

$\mathrm{RR} \mathrm{n}^{\circ} 8670$ 
For $\alpha_{1}=0$, we consider the Lagrange interpolant $\boldsymbol{I}_{h}^{\Omega^{\mathrm{s}}}$ onto $\boldsymbol{V}_{h}^{\mathrm{s}}$ for the solid velocity, while for the fluid velocity we consider the Stokes-like operator (see, e.g., [17]),

$$
\left(\boldsymbol{R}_{h}, R_{h}\right): \boldsymbol{V}^{\mathrm{f}} \rightarrow \boldsymbol{V}_{h}^{\mathrm{f}} \times Q_{h},
$$

defined as follows: for all $\boldsymbol{u} \in \boldsymbol{V}^{\mathrm{f}}$, the pair $\left(\boldsymbol{R}_{h} \boldsymbol{u}, R_{h} \boldsymbol{u}\right) \in \boldsymbol{V}_{h}^{\mathrm{f}} \times Q_{h}$ stands for the unique solution of the discrete Stokes problem

$$
\left\{\begin{array}{l}
\left.\boldsymbol{R}_{h} \boldsymbol{u}\right|_{\Sigma}=\boldsymbol{I}_{h}^{\Sigma}\left(\left.\boldsymbol{u}\right|_{\Sigma}\right) \\
a\left(\boldsymbol{R}_{h} \boldsymbol{u}, \boldsymbol{v}_{h}^{\mathrm{f}}\right)+b\left(R_{h} \boldsymbol{u}, \boldsymbol{v}_{h}^{\mathrm{f}}\right)=a\left(\boldsymbol{u}, \boldsymbol{v}_{h}^{\mathrm{f}}\right) \quad \forall \boldsymbol{v}_{h}^{\mathrm{f}} \in \boldsymbol{V}_{\Sigma, h}, \\
b\left(q_{h}, \boldsymbol{R}_{h} \boldsymbol{u}\right)=s_{h}\left(R_{h} \boldsymbol{u}, q_{h}\right) \quad \forall q_{h} \in Q_{h},
\end{array}\right.
$$

where $\boldsymbol{I}_{h}^{\Sigma}$ denotes the Lagrange interpolant onto the discrete trace space $\boldsymbol{\Lambda}_{\Sigma, h}$ given by (6). Owing to the nodal nature of the Lagrange interpolant, we have $\boldsymbol{I}_{h}^{\Sigma}\left(\left.\dot{\boldsymbol{d}}\right|_{\Sigma}\right)=\left.\left(\boldsymbol{I}_{h}^{\Omega^{\mathrm{s}}} \dot{\boldsymbol{d}}\right)\right|_{\Sigma}$. Hence, from $(36)_{1}$, we get

$$
\boldsymbol{R}_{h} \boldsymbol{u}=\boldsymbol{I}_{h}^{\Omega^{\mathrm{s}}} \dot{\boldsymbol{d}} \text { on } \Sigma,
$$

or, equivalently, $\left(\boldsymbol{R}_{h} \boldsymbol{u}, \boldsymbol{I}_{h}^{\Omega^{\mathrm{s}}} \dot{\boldsymbol{d}}\right) \in \boldsymbol{W}_{h}$.

Standard interpolation theory (see, .e.g, [5, Chapter 4]) yields the estimates

$$
\begin{aligned}
\left\|\dot{\boldsymbol{d}}-\boldsymbol{I}_{h}^{\Omega^{\mathrm{s}}} \dot{\boldsymbol{d}}\right\|_{0, \Omega^{\mathrm{s}}}+h\left\|\dot{\boldsymbol{d}}-\boldsymbol{I}_{h}^{\Omega^{\mathrm{s}}} \dot{\boldsymbol{d}}\right\|_{1, \Omega^{\mathrm{s}}} & \lesssim h^{2}\|\dot{\boldsymbol{d}}\|_{2, \Omega^{\mathrm{s}}}, \\
\left\|\boldsymbol{I}_{h}^{\Omega^{\mathrm{s}}} \dot{\boldsymbol{d}}\right\|_{1, \Omega^{\mathrm{s}}} & \lesssim h\|\dot{\boldsymbol{d}}\|_{2, \Omega^{\mathrm{s}}}+\|\dot{\boldsymbol{d}}\|_{1, \Omega^{\mathrm{s}}}
\end{aligned}
$$

for $\dot{\boldsymbol{d}} \in\left[H^{2}\left(\Omega^{\mathrm{s}}\right)\right]^{d}$. On the other hand, from [17, Lemma 3], it follows that

$$
\begin{aligned}
\left\|\boldsymbol{u}-\boldsymbol{R}_{h} \boldsymbol{u}\right\|_{\boldsymbol{V}^{\mathrm{f}}}+\left|R_{h} \boldsymbol{u}\right|_{s_{h}}+\left\|R_{h} \boldsymbol{u}\right\|_{Q} & \lesssim h \mu^{\frac{1}{2}}\|\boldsymbol{u}\|_{2, \Omega^{\mathrm{f}}}, \\
\left\|\boldsymbol{u}-\boldsymbol{R}_{h} \boldsymbol{u}\right\|_{0, \Omega^{\mathrm{f}}} & \leq c_{\mu} \mu^{\frac{1}{2}} h^{2}\|\boldsymbol{u}\|_{2, \Omega^{\mathrm{f}}}+\tilde{c}_{\mu} h^{2}\|\boldsymbol{u}\|_{2, \Sigma},
\end{aligned}
$$

for all $\boldsymbol{u} \in\left[H^{2}\left(\Omega^{\mathrm{f}}\right)\right]^{d}$ with $\left.\boldsymbol{u}\right|_{\Sigma} \in\left[H^{2}(\Sigma)\right]^{d}$ and $\operatorname{div} \boldsymbol{u}=0$ in $\Omega^{\mathrm{f}}$.

For $\alpha_{1}>0$, we consider a coupled fluid-solid velocity projector,

$$
\left(\left(\boldsymbol{S}_{h}^{\mathrm{f}}, \boldsymbol{S}_{h}^{\mathrm{s}}\right), S_{h}\right): \boldsymbol{W} \longrightarrow \boldsymbol{W}_{h} \times Q_{h},
$$

defined as follows: For $(\boldsymbol{u}, \dot{\boldsymbol{d}}) \in \boldsymbol{W}$, we find $\left(\left(\boldsymbol{u}_{h}, \dot{\boldsymbol{d}}_{h}\right), p_{h}\right) \in \boldsymbol{W}_{h} \times Q_{h}$ such that

$$
\left\{\begin{array}{l}
a\left(\boldsymbol{u}_{h}, \boldsymbol{v}_{h}^{\mathrm{f}}\right)+\alpha_{1} a^{\mathrm{e}}\left(\dot{\boldsymbol{d}}_{h}, \boldsymbol{v}_{h}^{\mathrm{s}}\right)+b\left(p_{h}, \boldsymbol{v}_{h}^{\mathrm{f}}\right)=a\left(\boldsymbol{u}, \boldsymbol{v}_{h}^{\mathrm{f}}\right)+\alpha_{1} a^{\mathrm{e}}\left(\dot{\boldsymbol{d}}, \boldsymbol{v}_{h}^{\mathrm{s}}\right) \quad \forall\left(\boldsymbol{v}_{h}^{\mathrm{f}}, \boldsymbol{v}_{h}^{\mathrm{s}}\right) \in \boldsymbol{W}_{h}, \\
b\left(q_{h}, \boldsymbol{u}_{h}\right)=s_{h}\left(p_{h}, q_{h}\right) \quad \forall q_{h} \in Q_{h}
\end{array}\right.
$$

then we set

$$
\boldsymbol{S}_{h}^{\mathrm{f}}(\boldsymbol{u}, \dot{\boldsymbol{d}}) \stackrel{\text { def }}{=} \boldsymbol{u}_{h}, \quad \boldsymbol{S}_{h}^{\mathrm{s}}(\boldsymbol{u}, \dot{\boldsymbol{d}}) \stackrel{\text { def }}{=} \dot{\boldsymbol{d}}_{h}, \quad S_{h}(\boldsymbol{u}, \dot{\boldsymbol{d}}) \stackrel{\text { def }}{=} p_{h} .
$$

Note that, by construction, we have

$$
\boldsymbol{S}_{h}^{\mathrm{f}}(\boldsymbol{u}, \dot{\boldsymbol{d}})=\boldsymbol{S}_{h}^{\mathrm{s}}(\boldsymbol{u}, \dot{\boldsymbol{d}}) \quad \text { on } \quad \Sigma .
$$

The approximation properties of $\left(\left(\boldsymbol{S}_{h}^{\mathrm{f}}, \boldsymbol{S}_{h}^{\mathrm{s}}\right), S_{h}\right)$ are stated in the next result. 
Lemma 3 Let $(\boldsymbol{u}, \dot{\boldsymbol{d}}) \in \boldsymbol{W} \cap\left(\left[H^{2}\left(\Omega^{\mathrm{f}}\right)\right]^{d} \times\left[H^{2}\left(\Omega^{\mathrm{s}}\right)\right]^{d}\right)$ be given with divu $=0$ in $\Omega^{\mathrm{f}}$. The following estimates hold:

$$
\begin{array}{r}
\left\|\boldsymbol{S}_{h}^{\mathrm{f}}(\boldsymbol{u}, \dot{\boldsymbol{d}})\right\|_{\boldsymbol{V}^{\mathrm{f}}}+\alpha_{1}^{\frac{1}{2}}\left\|\boldsymbol{S}_{h}^{\mathrm{s}}(\boldsymbol{u}, \dot{\boldsymbol{d}})\right\|_{\mathrm{e}}+\left|S_{h}(\boldsymbol{u}, \dot{\boldsymbol{d}})\right|_{s_{h}} \\
\left\|\boldsymbol{u}-\boldsymbol{S}_{h}^{\mathrm{f}}\left(\boldsymbol{u}\left\|_{\boldsymbol{V}^{\mathrm{f}}}+\alpha_{1}^{\frac{1}{2}}\right\| \dot{\boldsymbol{d}}\right)\right\|_{V^{\mathrm{f}}}+\alpha_{1}^{\frac{1}{2}}\left\|\dot{\boldsymbol{d}}-\boldsymbol{S}_{h}^{\mathrm{s}}(\boldsymbol{u}, \dot{\boldsymbol{d}})\right\|_{\mathrm{e}}+\left\|S_{h}(\boldsymbol{u}, \dot{\boldsymbol{d}})\right\|_{Q}+\left|S_{h}(\boldsymbol{u}, \dot{\boldsymbol{d}})\right|_{s_{h}} \\
\lesssim h \mu^{\frac{1}{2}}\|\boldsymbol{u}\|_{2, \Omega^{\mathrm{f}}} \\
+h\left(\alpha_{1} \beta_{\mathrm{e}}\right)^{\frac{1}{2}}\|\dot{\boldsymbol{d}}\|_{2, \Omega^{\mathrm{s}}} .
\end{array}
$$

Proof 3 See Section A of the appendix.

Owing to the above discussion, for the fluid and solid velocities we consider the projection operator,

$$
\left(\left(\boldsymbol{P}_{h}^{\mathrm{f}}, \boldsymbol{P}_{h}^{\mathrm{s}}\right), P_{h}\right): \boldsymbol{W} \longrightarrow \boldsymbol{W}_{h} \times Q_{h},
$$

defined as,

$$
\begin{aligned}
& \boldsymbol{P}_{h}^{\mathrm{f}}(\boldsymbol{u}, \dot{\boldsymbol{d}}) \stackrel{\text { def }}{=}\left\{\begin{array} { l l l } 
{ \boldsymbol { R } _ { h } \boldsymbol { u } } & { \text { if } } & { \alpha _ { 1 } = 0 , } \\
{ \boldsymbol { S } _ { h } ^ { \mathrm { f } } ( \boldsymbol { u } , \dot { \boldsymbol { d } } ) } & { \text { if } } & { \alpha _ { 1 } > 0 , }
\end{array} \quad \boldsymbol { P } _ { h } ^ { \mathrm { s } } ( \boldsymbol { u } , \dot { \boldsymbol { d } } ) \stackrel { \text { def } } { = } \left\{\begin{array}{lll}
\boldsymbol{I}_{h}^{\Omega^{\mathrm{s}}} \dot{\boldsymbol{d}} & \text { if } & \alpha_{1}=0, \\
\boldsymbol{S}_{h}^{\mathrm{s}}(\boldsymbol{u}, \dot{\boldsymbol{d}}) & \text { if } & \alpha_{1}>0,
\end{array}\right.\right. \\
& P_{h}(\boldsymbol{u}, \dot{\boldsymbol{d}}) \stackrel{\text { def }}{=}\left\{\begin{array}{lll}
R_{h} \boldsymbol{u} & \text { if } & \alpha_{1}=0, \\
S_{h}(\boldsymbol{u}, \dot{\boldsymbol{d}}) & \text { if } & \alpha_{1}>0,
\end{array}\right.
\end{aligned}
$$

for all $(\boldsymbol{u}, \dot{d}) \in W$.

\subsection{Error decomposition}

In order to ease the presentation, we will use the notation $y^{n}$ (without subscript ${ }_{h}$ ) to denote the temporal value $y\left(t_{n}\right)$ of a time dependent function $x$ (that can be, e.g., $\boldsymbol{d}, \dot{\boldsymbol{d}}, \boldsymbol{u}$ or $p$ ). Furthermore, we will frequently make a convenient abuse of notation which consists in using $\partial_{t} y^{n}$ instead of $\left(\partial_{t} y\right)^{n}$. This also applies to other operators acting on $y$.

We split the solid displacement error at the time instant $t_{n}$ as:

$$
\boldsymbol{d}^{n}-\boldsymbol{d}_{h}^{n}=\underbrace{\boldsymbol{d}^{n}-\boldsymbol{\pi}_{h}^{\mathrm{e}} \boldsymbol{d}^{n}}_{\stackrel{\text { def }}{=} \boldsymbol{\xi}_{\pi}^{n}}+\underbrace{\boldsymbol{\pi}_{h}^{\mathrm{e}} \boldsymbol{d}^{n}-\boldsymbol{d}_{h}^{n}}_{\stackrel{\text { def }}{=} \boldsymbol{\xi}_{h}^{n}} .
$$

For the fluid pressure, we take

$$
p^{n}-p_{h}^{n}=\underbrace{p^{n}-\Pi_{h} p^{n}}_{\stackrel{\text { def }}{=} y_{\pi}^{n}}+\underbrace{\Pi_{h} p^{n}-p_{h}^{n}}_{\stackrel{\text { def }}{=} y_{h}^{n}} .
$$

At last, the fluid and solid velocity errors are decomposed as

$\mathrm{RR} \mathrm{n}^{\circ} 8670$

$$
\begin{gathered}
\boldsymbol{u}^{n}-\boldsymbol{u}_{h}^{n}=\underbrace{\boldsymbol{u}^{n}-\boldsymbol{P}_{h}^{\mathrm{f}}\left(\boldsymbol{u}^{n}, \dot{\boldsymbol{d}}^{n}\right)}_{\stackrel{\text { def }}{=} \boldsymbol{\theta}_{\pi}^{n}}+\underbrace{\boldsymbol{P}_{h}^{\mathrm{f}}\left(\boldsymbol{u}^{n}, \dot{\boldsymbol{d}}^{n}\right)-\boldsymbol{u}_{h}^{n}}_{\stackrel{\text { def }}{=} \boldsymbol{\theta}_{h}^{n}}, \\
\dot{\boldsymbol{d}}^{n}-\dot{\boldsymbol{d}}_{h}^{n}=\underbrace{\dot{\boldsymbol{d}}^{n}-\boldsymbol{P}_{h}^{\mathrm{s}}\left(\boldsymbol{u}^{n}, \dot{\boldsymbol{d}}^{n}\right)}_{\stackrel{\text { def }}{=} \dot{\boldsymbol{\xi}}_{\pi}^{n}}+\underbrace{\boldsymbol{P}_{h}^{\mathrm{s}}\left(\boldsymbol{u}^{n}, \dot{\boldsymbol{d}}^{n}\right)-\dot{\boldsymbol{d}}_{h}^{n}}_{\stackrel{\text { def }}{=} \dot{\boldsymbol{\xi}}_{h}^{n}} .
\end{gathered}
$$


We conclude this section with two fundamental results, which will be useful for the proofs of the discrete error estimates stated in Section 4.4. The first has to do with the fact that the quantities $\boldsymbol{\theta}_{h}^{n}$ and $\dot{\boldsymbol{\xi}}_{h}^{n}$, introduced in (47), do not match at the interface. In other words, the pair $\left(\boldsymbol{\theta}_{h}^{n}, \dot{\boldsymbol{\xi}}_{h}^{n}\right)$ does not belong to $\boldsymbol{W}_{h}$. The next Lemma shows how to circumvent this via suitable corrections.

Lemma 4 Let $\dot{\boldsymbol{\xi}}_{h}^{n-\frac{1}{2}} \in \boldsymbol{V}_{h}^{\mathrm{s}}$ be defined as

$$
\begin{aligned}
\dot{\boldsymbol{\xi}}_{h}^{n-\frac{1}{2}} \stackrel{\text { def }}{=} \dot{\boldsymbol{\xi}}_{h}^{n}+ & \frac{\tau}{\rho^{\mathrm{s}}}\left[\boldsymbol{L}_{h}^{\mathrm{e}}\left(\boldsymbol{\xi}_{h}^{n}-\boldsymbol{\xi}_{h}^{n, \star}\right)+\boldsymbol{L}_{h}^{\mathrm{v}}\left(\dot{\boldsymbol{\xi}}_{h}^{n}-\dot{\boldsymbol{\xi}}_{h}^{n, \star}\right)\right] \\
& -\frac{\tau}{\rho^{\mathrm{s}}}\left[\boldsymbol{L}_{h}^{\mathrm{e}}\left(\boldsymbol{d}^{n}-\boldsymbol{d}^{n, \star}\right)+\boldsymbol{L}_{h}^{\mathrm{v}} \boldsymbol{P}_{h}^{\mathrm{s}}\left(\boldsymbol{u}^{n}-\boldsymbol{u}^{n, \star}, \dot{\boldsymbol{d}}^{n}-\dot{\boldsymbol{d}}^{n, \star}\right)\right] .
\end{aligned}
$$

Then, we have $\boldsymbol{\theta}_{h}^{n}=\dot{\boldsymbol{\xi}}_{h}^{n-\frac{1}{2}}$ on $\Sigma$.

Proof 4 The interface identity follows from (17), (37), (41), (45) and (47).

The next Lemma is motivated by the observation that, even though the standard displacementvelocity relation $\dot{\boldsymbol{d}}_{h}^{n}=\partial_{\tau} \boldsymbol{d}_{h}^{n}$ holds for Algorithm 1 and 2, their discrete error counterparts, $\dot{\boldsymbol{\xi}}_{h}^{n}$ and $\partial_{\tau} \boldsymbol{\xi}_{h}^{n}$, do not satisfy a similar expression. Basically, this comes from the fact that different projection operators are considered for the solid unknowns in (45) and (47) 2 . More precisely, we have the following result.

Lemma 5 Let $\boldsymbol{z}_{h}^{n} \in \boldsymbol{V}_{h}^{\mathrm{s}}$ be defined as

$$
\boldsymbol{z}_{h}^{n} \stackrel{\text { def }}{=} \dot{\boldsymbol{\xi}}_{h}^{n}-\partial_{\tau} \boldsymbol{\xi}_{h}^{n} .
$$

There holds

$$
\boldsymbol{z}_{h}^{n}=\boldsymbol{P}_{h}^{\mathrm{s}}\left(\boldsymbol{u}^{n}, \dot{\boldsymbol{d}}^{n}\right)-\boldsymbol{\pi}_{h}^{\mathrm{e}} \partial_{\tau} \boldsymbol{d}^{n}
$$

and

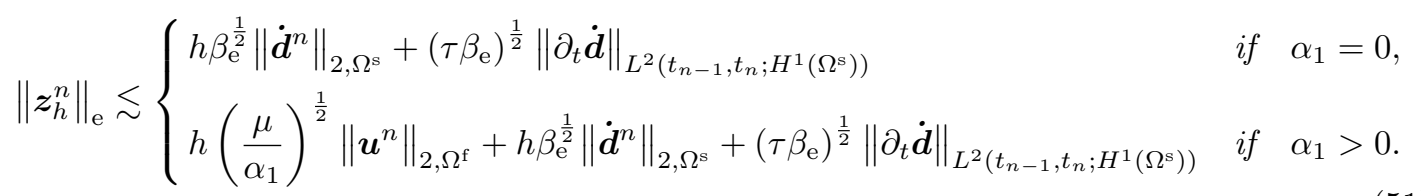

Proof 5 The identity (50) follows directly from (18), (45) and (47) $)_{2}$. With regard to the estimate (51), by adding and subtracting suitable terms and using $(2)_{2}$, we have

$$
\left\|\boldsymbol{z}_{h}^{n}\right\|_{\mathrm{e}} \leq\left\|\boldsymbol{P}_{h}^{\mathrm{s}}\left(\boldsymbol{u}^{n}, \dot{\boldsymbol{d}}^{n}\right)-\dot{\boldsymbol{d}}^{n}\right\|_{\mathrm{e}}+\left\|\dot{\boldsymbol{d}}^{n}-\boldsymbol{\pi}_{h}^{\mathrm{e}} \dot{\boldsymbol{d}}^{n}\right\|_{\mathrm{e}}+\left\|\boldsymbol{\pi}_{h}^{\mathrm{e}}\left(\partial_{t}-\partial_{\tau}\right) \boldsymbol{d}^{n}\right\|_{\mathrm{e}}
$$

and we conclude by estimating each term separately using (33), (43) and a Taylor expansion.

\subsection{Discrete error estimation}

For $n>r$, we define the energy norm of the discrete discrete error at time $t_{n}$ as

$$
\begin{aligned}
\mathcal{Z}_{h}^{n} \stackrel{\text { def }}{=}\left(\rho^{\mathrm{f}}\right)^{\frac{1}{2}}\left\|\boldsymbol{\theta}_{h}^{n}\right\|_{0, \Omega^{\mathrm{f}}}+\left(\sum_{m=r+1}^{n} \tau\left\|\boldsymbol{\theta}_{h}^{m}\right\|_{\boldsymbol{V}}^{2}\right)^{\frac{1}{2}} & +\left(\sum_{m=r+1}^{n} \tau\left|y_{h}^{m}\right|_{s_{h}}^{2}\right)^{\frac{1}{2}} \\
& +\rho^{\mathrm{s} \frac{1}{2}}\left\|\dot{\boldsymbol{\xi}}_{h}^{n}\right\|_{0, \Omega^{\mathrm{s}}}+\left\|\boldsymbol{\xi}_{h}^{n}\right\|_{\mathrm{e}}+\left(\sum_{m=r+1}^{n} \tau\left\|\dot{\boldsymbol{\xi}}_{h}^{m}\right\|_{\mathrm{v}}^{2}\right)^{\frac{1}{2}} .
\end{aligned}
$$


This section states a bound for $\mathcal{Z}_{h}^{n}$. To this purpose, we make the following assumptions on the exact solution $(\boldsymbol{u}, p, \boldsymbol{d}, \dot{\boldsymbol{d}})$ of the continuous problem $(5)$ :

- $\alpha_{1}=0$ :

$$
\begin{aligned}
& \boldsymbol{u} \in\left[H^{1}\left(0, T ; H^{2}\left(\Omega^{\mathrm{f}}\right)\right)\right]^{d}, \quad \partial_{t t} \boldsymbol{u} \in\left[L^{2}\left(0, T ; L^{2}\left(\Omega^{\mathrm{f}}\right)\right)\right]^{d},\left.\quad \boldsymbol{u}\right|_{\Sigma} \in\left[H^{1}\left(0, T ; H^{2}(\Sigma)\right)\right]^{d}, \\
& p \in H^{1}\left(0, T ; H^{1}\left(\Omega^{\mathrm{f}}\right)\right), \quad \dot{\boldsymbol{d}} \in\left[H^{2}\left(0, T ; H^{2}\left(\Omega^{\mathrm{s}}\right)\right)\right]^{d} ;
\end{aligned}
$$

- $\alpha_{1}>0$ :

$$
\begin{array}{lll}
\boldsymbol{u} \in\left[H^{1}\left(0, T ; H^{2}\left(\Omega^{\mathrm{f}}\right)\right)\right]^{d}, & \partial_{t t} \boldsymbol{u} \in\left[L^{2}\left(0, T ; H^{1}\left(\Omega^{\mathrm{f}}\right)\right)\right]^{d}, & p \in H^{1}\left(0, T ; H^{1}\left(\Omega^{\mathrm{f}}\right)\right), \\
\dot{\boldsymbol{d}} \in\left[H^{1}\left(0, T ; H^{2}\left(\Omega^{\mathrm{s}}\right)\right)\right]^{d}, & \partial_{t t} \dot{\boldsymbol{d}} \in\left[L^{2}\left(0, T ; H^{1}\left(\Omega^{\mathrm{s}}\right)\right)\right]^{d}
\end{array}
$$

The next theorem states an a priori estimate for the discrete error $\mathcal{Z}_{h}^{n}$.

Theorem 6 Let $(\boldsymbol{u}, p, \boldsymbol{d}, \dot{\boldsymbol{d}})$ be the solution of the coupled problem (5) and

$$
\left\{\left(\boldsymbol{u}_{h}^{n}, p_{h}^{n}, \boldsymbol{d}_{h}^{n}, \dot{\boldsymbol{d}}_{h}^{n}\right)\right\}_{n>r}
$$

be the discrete solution given by Algorithm 1 or 2 with

$$
\left(\boldsymbol{u}_{h}^{0}, \boldsymbol{d}_{h}^{0}, \dot{\boldsymbol{d}}_{h}^{0}\right)=\left(\boldsymbol{P}_{h}^{\mathrm{f}}\left(\boldsymbol{u}^{0}, \dot{\boldsymbol{d}}^{0}\right), \boldsymbol{\pi}_{h}^{\mathrm{e}} \boldsymbol{d}^{0}, \boldsymbol{P}_{h}^{\mathrm{s}}\left(\boldsymbol{u}^{0}, \dot{\boldsymbol{d}}^{0}\right)\right)
$$

and, depending on the extrapolation order $r \in\{0,1,2\}$, with the initiation procedure detailed in Remark 5. We assume that the exact solution has the regularity provided by (52)-(53) and that

$$
\left(\alpha_{0}+1 / T\right) \tau<1 \text {. }
$$

For the schemes with $r=2$ we assume, in addition, that the stability condition (24) holds with

$$
\frac{\gamma \tau^{2}}{T}+2 \delta^{2}<1
$$

Then, the following error estimate holds, for $n>r$ such that $n \tau<T$ :

$$
\mathcal{Z}_{h}^{n} \lesssim \begin{cases}c_{1} h+c_{2} \tau+c_{3} \tau^{2^{r-1}}\left\|\boldsymbol{L}_{h}^{\mathrm{e}} \boldsymbol{d}+\boldsymbol{L}_{h}^{\mathrm{v}} \boldsymbol{P}_{h}^{\mathrm{s}}(\boldsymbol{u}, \dot{\boldsymbol{d}})\right\|_{H^{r}\left(0, T ; L^{2}\left(\Omega^{\mathrm{s}}\right)\right)} & \text { if } \quad \alpha_{1}=0, \\ \left(c_{4}+c_{5} \alpha_{1}^{-\frac{1}{2}}\right) h+c_{6} \tau+c_{7} \tau^{2^{r-1}}\left\|\boldsymbol{L}_{h}^{\mathrm{e}} \boldsymbol{d}+\boldsymbol{L}_{h}^{\mathrm{v}} \boldsymbol{P}_{h}^{\mathrm{s}}(\boldsymbol{u}, \dot{\boldsymbol{d}})\right\|_{H^{r}\left(0, T ; L^{2}\left(\Omega^{\mathrm{s}}\right)\right)} & \text { if } \quad \alpha_{1}>0 .\end{cases}
$$

Here, the symbols $\left\{c_{i}\right\}_{i=1}^{7}$ denote positive constants independent of $h$ and $\tau$, but which depend on the extrapolation order $r$, on the physical parameters and on the regularity of the exact solution $(\boldsymbol{u}, p, \boldsymbol{d}, \dot{\boldsymbol{d}})$.

Proof 6 Basically, the proof follows the same steps as in the case of the coupling with a thinwalled structure (see [17, 20]), but with further complications arising from:

- the distributed nature of the solid velocity, viz., we cannot control the solid velocity contributions in terms of the fluid viscous dissipation (terms $T_{2}, T_{3,2}, T_{4}, T_{8}$ and $T_{9}$ below);

- the different velocity projection operators (terms $T_{3,1}$ and $T_{3,2}$ below);

- the lumped-mass approximation in the thick-walled solid (term $T_{4}$ below).

$\mathrm{RR} \mathrm{n}^{\circ} 8670$ 
From (5) and (18) we get the following modified Galerkin orthogonality

$$
\begin{aligned}
\rho^{\mathrm{f}}\left(\partial_{\tau}\left(\boldsymbol{u}^{n}-\boldsymbol{u}_{h}^{n}\right), \boldsymbol{v}_{h}^{\mathrm{f}}\right)_{\Omega^{\mathrm{f}}}+a( & \left(\boldsymbol{u}^{n}-\boldsymbol{u}_{h}^{n}, \boldsymbol{v}_{h}^{\mathrm{f}}\right)+b\left(p^{n}-p_{h}^{n}, \boldsymbol{v}_{h}^{\mathrm{f}}\right)-b\left(q_{h}, \boldsymbol{u}^{n}-\boldsymbol{u}_{h}^{n}\right) \\
+\rho^{\mathrm{s}}\left(\partial_{\tau} \dot{\boldsymbol{d}}^{n}, \boldsymbol{v}_{h}^{\mathrm{s}}\right)_{\Omega^{\mathrm{s}}} & -\rho^{\mathrm{s}}\left(\partial_{\tau} \dot{\boldsymbol{d}}_{h}^{n}, \boldsymbol{v}_{h}^{\mathrm{s}}\right)_{*}+a^{\mathrm{e}}\left(\boldsymbol{d}^{n}-\boldsymbol{d}_{h}^{n}, \boldsymbol{v}_{h}^{\mathrm{s}}\right)+a^{\mathrm{v}}\left(\dot{\boldsymbol{d}}^{n}, \boldsymbol{v}_{h}^{\mathrm{s}}\right)-a_{*}^{\mathrm{v}}\left(\dot{\boldsymbol{d}}_{h}^{n}, \boldsymbol{v}_{h}^{\mathrm{s}}\right) \\
& =\rho^{\mathrm{f}}\left(\left(\partial_{\tau}-\partial_{t}\right) \boldsymbol{u}^{n}, \boldsymbol{v}_{h}^{\mathrm{f}}\right)_{\Omega^{\mathrm{f}}}+\rho^{\mathrm{s}}\left(\left(\partial_{\tau}-\partial_{t}\right) \dot{\boldsymbol{d}}^{n}, \boldsymbol{v}_{h}^{\mathrm{s}}\right)_{\Omega^{\mathrm{s}}}+s_{h}\left(p_{h}^{n}, q_{h}\right)
\end{aligned}
$$

for all $\left(\left(\boldsymbol{v}_{h}^{\mathrm{f}}, \boldsymbol{v}_{h}^{\mathrm{s}}\right), q_{h}\right) \in \boldsymbol{W}_{h} \times Q_{h}$ and $n>r$. On the other hand, using (47), we have

$$
\begin{aligned}
\rho^{\mathrm{s}}\left(\partial_{\tau} \dot{\boldsymbol{d}}^{n}, \boldsymbol{v}_{h}^{\mathrm{s}}\right)_{\Omega^{\mathrm{s}}}-\rho^{\mathrm{s}}\left(\partial_{\tau} \dot{\boldsymbol{d}}_{h}^{n}, \boldsymbol{v}_{h}^{\mathrm{s}}\right)_{*} & =\rho^{\mathrm{s}}\left(\partial_{\tau} \dot{\boldsymbol{\xi}}_{h}^{n}, \boldsymbol{v}_{h}^{\mathrm{s}}\right)_{*}+\rho^{\mathrm{s}}\left(\partial_{\tau} \dot{\boldsymbol{\xi}}_{\pi}^{n}, \boldsymbol{v}_{h}^{\mathrm{s}}\right)_{\Omega^{\mathrm{s}}}+\rho^{\mathrm{s}} \delta_{h}\left(\partial_{\tau} \boldsymbol{P}_{h}^{\mathrm{s}}\left(\boldsymbol{u}^{n}, \dot{\boldsymbol{d}}^{n}\right), \boldsymbol{v}_{h}^{\mathrm{s}}\right), \\
a^{\mathrm{v}}\left(\dot{\boldsymbol{d}}^{n}, \boldsymbol{v}_{h}^{\mathrm{s}}\right)-a_{*}^{\mathrm{v}}\left(\dot{\boldsymbol{d}}_{h}^{n}, \boldsymbol{v}_{h}^{\mathrm{s}}\right) & =a_{*}^{\mathrm{v}}\left(\dot{\boldsymbol{\xi}}_{h}^{n}, \boldsymbol{v}_{h}^{\mathrm{s}}\right)+a^{\mathrm{v}}\left(\dot{\boldsymbol{\xi}}_{\pi}^{n}, \boldsymbol{v}_{h}^{\mathrm{s}}\right)+\alpha_{0} \rho^{\mathrm{s}} \delta_{h}\left(\boldsymbol{P}_{h}^{\mathrm{s}}\left(\boldsymbol{u}^{n}, \dot{\boldsymbol{d}}^{n}\right), \boldsymbol{v}_{h}^{\mathrm{s}}\right)
\end{aligned}
$$

for all $\boldsymbol{v}_{h}^{\mathrm{s}} \in \boldsymbol{V}_{h}^{\mathrm{s}}$. Hence, by inserting this expression into (57) and using the remaining error decompositions (45)-(47), we infer that

$$
\begin{aligned}
& \rho^{\mathrm{f}}\left(\partial_{\tau} \boldsymbol{\theta}_{h}^{n}, \boldsymbol{v}_{h}^{\mathrm{f}}\right)_{\Omega^{\mathrm{f}}}+a\left(\boldsymbol{\theta}_{h}^{n}, \boldsymbol{v}_{h}^{\mathrm{f}}\right)+b\left(y_{h}^{n}, \boldsymbol{v}_{h}^{\mathrm{f}}\right)-b\left(q_{h}, \boldsymbol{\theta}_{h}^{n}\right)+s_{h}\left(y_{h}^{n}, q_{h}\right)+\rho^{\mathrm{s}}\left(\partial_{\tau} \dot{\boldsymbol{\xi}}_{h}^{n}, \boldsymbol{v}_{h}^{\mathrm{s}}\right)_{*}+a^{\mathrm{e}}\left(\boldsymbol{\xi}_{h}^{n}, \boldsymbol{v}_{h}^{\mathrm{s}}\right) \\
& +a_{*}^{\mathrm{v}}\left(\dot{\boldsymbol{\xi}}_{h}^{n}, \boldsymbol{v}_{h}^{\mathrm{s}}\right)=\underbrace{\rho^{\mathrm{f}}\left(\left(\partial_{\tau}-\partial_{t}\right) \boldsymbol{u}^{n}, \boldsymbol{v}_{h}^{\mathrm{f}}\right)_{\Omega^{\mathrm{f}}}-\rho^{\mathrm{f}}\left(\partial_{\tau} \boldsymbol{\theta}_{\pi}^{n}, \boldsymbol{v}_{h}^{\mathrm{f}}\right)_{\Omega^{\mathrm{f}}}}_{\stackrel{\text { def }}{=} T_{1}\left(\boldsymbol{v}_{h}^{\mathrm{f}}\right)} \\
& \underbrace{+\rho^{\mathrm{s}}\left(\left(\partial_{\tau}-\partial_{t}\right) \dot{\boldsymbol{d}}^{n}, \boldsymbol{v}_{h}^{\mathrm{s}}\right)_{\Omega^{\mathrm{s}}}-\rho^{\mathrm{s}}\left(\partial_{\tau} \dot{\boldsymbol{\xi}}_{\pi}^{n}, \boldsymbol{v}_{h}^{\mathrm{s}}\right)_{\Omega^{\mathrm{s}}}}_{\stackrel{\text { def }}{=} T_{2}\left(\boldsymbol{v}_{h}^{\mathrm{s}}\right)} \\
& \underbrace{-a\left(\boldsymbol{\theta}_{\pi}^{n}, \boldsymbol{v}_{h}^{\mathrm{f}}\right)-b\left(y_{\pi}^{n}, \boldsymbol{v}_{h}^{\mathrm{f}}\right)+b\left(q_{h}, \boldsymbol{\theta}_{\pi}^{n}\right)+s_{h}\left(\Pi_{h} p^{n}, q_{h}\right)}_{\stackrel{\text { def }}{=} T_{3,1}\left(\boldsymbol{v}_{h}^{\mathrm{f}}, q_{h}\right)}+\underbrace{a^{\mathrm{v}}\left(\dot{\boldsymbol{\xi}}_{\pi}^{n}, \boldsymbol{v}_{h}^{\mathrm{s}}\right)}_{\stackrel{\text { def }}{=} T_{3,2}\left(\boldsymbol{v}_{h}^{\mathrm{s}}\right)} \\
& +\underbrace{\rho^{\mathrm{s}} \delta_{h}\left(\left(\partial_{\tau}+\alpha_{0}\right) \boldsymbol{P}_{h}^{\mathrm{s}}\left(\boldsymbol{u}^{n}, \dot{\boldsymbol{d}}^{n}\right), \boldsymbol{v}_{h}^{\mathrm{s}}\right)}_{\stackrel{\text { def }}{=} T_{4}\left(\boldsymbol{v}_{h}^{\mathrm{s}}\right)}+\underbrace{a^{\mathrm{e}}\left(\boldsymbol{\xi}_{\pi}^{n}, \boldsymbol{v}_{h}^{\mathrm{s}}\right)}_{=0}
\end{aligned}
$$

for all $\left(\left(\boldsymbol{v}_{h}^{\mathrm{f}}, \boldsymbol{v}_{h}^{\mathrm{s}}\right), q_{h}\right) \in \boldsymbol{W}_{h} \times Q_{h}$ and $n>r$.

Owing to Lemma 4, we can safely test (58) with $\left(\boldsymbol{v}_{h}^{\mathrm{f}}, \boldsymbol{v}_{h}^{\mathrm{s}}\right)=\tau\left(\boldsymbol{\theta}_{h}^{n}, \dot{\boldsymbol{\xi}}^{n-\frac{1}{2}}\right), q_{h}=\tau y_{h}^{n}$ and then use (49) to get the following discrete energy error identity:

$$
\begin{aligned}
\frac{\rho^{\mathrm{f}}}{2}( & \left.\left\|\boldsymbol{\theta}_{h}^{n}\right\|_{0, \Omega^{\mathrm{f}}}^{2}-\left\|\boldsymbol{\theta}_{h}^{n-1}\right\|_{0, \Omega^{\mathrm{f}}}^{2}+\left\|\boldsymbol{\theta}_{h}^{n}-\boldsymbol{\theta}_{h}^{n-1}\right\|_{0, \Omega^{\mathrm{f}}}^{2}\right)+2 \mu \tau\left\|\boldsymbol{\epsilon}\left(\boldsymbol{\theta}_{h}^{n}\right)\right\|_{0, \Omega^{\mathrm{f}}}^{2}+\tau\left|y_{h}^{n}\right|_{\mathrm{s}, h}^{2} \\
& +\frac{\rho^{\mathrm{s}}}{2}\left(\left\|\dot{\boldsymbol{\xi}}_{h}^{n}\right\|_{*}^{2}-\left\|\dot{\boldsymbol{\xi}}_{h}^{n-1}\right\|_{*}^{2}+\left\|\dot{\boldsymbol{\xi}}_{h}^{n}-\dot{\boldsymbol{\xi}}_{h}^{n-1}\right\|_{*}^{2}\right)+\frac{1}{2}\left(\left\|\boldsymbol{\xi}_{h}^{n}\right\|_{\mathrm{e}}^{2}-\left\|\boldsymbol{\xi}_{h}^{n-1}\right\|_{\mathrm{e}}^{2}+\left\|\boldsymbol{\xi}_{h}^{n}-\boldsymbol{\xi}_{h}^{n-1}\right\|_{\mathrm{e}}^{2}\right) \\
& +\tau\left\|\dot{\boldsymbol{\xi}}_{h}^{n}\right\|_{\mathrm{v}, *}^{2}+T_{5,1}+T_{5,2} \\
= & T_{1}\left(\boldsymbol{v}_{h}^{\mathrm{f}}\right)+T_{2}\left(\boldsymbol{v}_{h}^{\mathrm{s}}\right)+T_{3,1}\left(\boldsymbol{v}_{h}^{\mathrm{f}}, q_{h}\right)+T_{3,2}\left(\boldsymbol{v}_{h}^{\mathrm{s}}\right)+T_{4}\left(\boldsymbol{v}_{h}^{\mathrm{s}}\right)+T_{6,1}+T_{6,2}+\underbrace{\tau a^{\mathrm{e}}\left(\boldsymbol{\xi}_{h}^{n}, \boldsymbol{z}_{h}^{n}\right)}_{\stackrel{\text { def }}{=} T_{7}} .
\end{aligned}
$$

The terms $T_{5,1}, T_{5,2}$ and $T_{6,1}, T_{6,2}$ correspond to the last two contributions of $\dot{\boldsymbol{\xi}}_{h}^{n-\frac{1}{2}}$ in (48), 
namely,

$$
\begin{aligned}
& T_{5,1} \stackrel{\text { def }}{=} \tau\left(\dot{\boldsymbol{\xi}}_{h}^{n}-\dot{\boldsymbol{\xi}}_{h}^{n-1}, \boldsymbol{L}_{h}^{\mathrm{e}}\left(\boldsymbol{\xi}_{h}^{n}-\boldsymbol{\xi}_{h}^{n, \star}\right)+\boldsymbol{L}_{h}^{\mathrm{v}}\left(\dot{\boldsymbol{\xi}}_{h}^{n}-\dot{\boldsymbol{\xi}}_{h}^{n, \star}\right)\right)_{*}, \\
& T_{5,2} \stackrel{\text { def }}{=} \frac{\tau^{2}}{\rho^{\mathrm{s}}}\left(\boldsymbol{L}_{h}^{\mathrm{e}} \boldsymbol{\xi}_{h}^{n}+\boldsymbol{L}_{h}^{\mathrm{v}} \dot{\boldsymbol{\xi}}_{h}^{n}, \boldsymbol{L}_{h}^{\mathrm{e}}\left(\boldsymbol{\xi}_{h}^{n}-\boldsymbol{\xi}_{h}^{n, \star}\right)+\boldsymbol{L}_{h}^{\mathrm{v}}\left(\dot{\boldsymbol{\xi}}_{h}^{n}-\dot{\boldsymbol{\xi}}_{h}^{n, \star}\right)\right)_{*}, \\
& T_{6,1} \stackrel{\text { def }}{=} \tau\left(\dot{\boldsymbol{\xi}}_{h}^{n}-\dot{\boldsymbol{\xi}}_{h}^{n-1}, \boldsymbol{L}_{h}^{\mathrm{e}}\left(\boldsymbol{d}^{n}-\boldsymbol{d}^{n, \star}\right)+\boldsymbol{L}_{h}^{\mathrm{v}} \boldsymbol{P}_{h}^{\mathrm{s}}\left(\boldsymbol{u}^{n}-\boldsymbol{u}^{n, \star}, \dot{\boldsymbol{d}}^{n}-\dot{\boldsymbol{d}}^{n, \star}\right)\right)_{*}, \\
& T_{6,2} \stackrel{\text { def }}{=} \frac{\tau^{2}}{\rho^{\mathrm{s}}}\left(\boldsymbol{L}_{h}^{\mathrm{e}} \boldsymbol{\xi}_{h}^{n}+\boldsymbol{L}_{h}^{\mathrm{v}} \dot{\boldsymbol{\xi}}_{h}^{n}, \boldsymbol{L}_{h}^{\mathrm{e}}\left(\boldsymbol{d}^{n}-\boldsymbol{d}^{n, \star}\right)+\boldsymbol{L}_{h}^{\mathrm{v}} \boldsymbol{P}_{h}^{\mathrm{s}}\left(\boldsymbol{u}^{n}-\boldsymbol{u}^{n, \star}, \dot{\boldsymbol{d}}^{n}-\dot{\boldsymbol{d}}^{n, \star}\right)\right)_{*} .
\end{aligned}
$$

In the last two terms, we have applied the identity $\boldsymbol{L}_{h}^{\mathrm{e}} \boldsymbol{\pi}_{h}^{\mathrm{e}}=\boldsymbol{L}_{h}^{\mathrm{e}}$ which can straightforwardly be inferred from (8) and (32).

The contributions given in (60) will be estimated below by distinguishing each case of extrapolation. The remain terms, $T_{1}, T_{2}, T_{3}, T_{4}$ and $T_{7}$ in (59), will be bounded irrespectively of $r$. Note that the term $T_{4}$ accounts for the error induced by the mass-lumping approximation in the solid. We proceed by estimating first the terms $T_{1}, T_{2}, T_{3}, T_{4}$ and $T_{7}$.

The treatment of the term $T_{1}$ is standard. Using the Poincaré inequality (with constant $C_{\mathrm{P}}$ ) and a Taylor expansion, we have

$$
\begin{aligned}
\left|T_{1}\left(\tau \boldsymbol{\theta}_{h}^{n}\right)\right| & \leq \rho^{\mathrm{f}} \tau\left(\left\|\left(\partial_{\tau}-\partial_{t}\right) \boldsymbol{u}^{n}\right\|_{0, \Omega^{\mathrm{f}}}+\left\|\partial_{\tau} \boldsymbol{\theta}_{\pi}^{n}\right\|_{0, \Omega^{\mathrm{f}}}\right)\left\|\boldsymbol{\theta}_{h}^{n}\right\|_{0, \Omega^{\mathrm{f}}} \\
& \leq \rho^{\mathrm{f}} \tau\left(\tau^{\frac{1}{2}}\left\|\partial_{t t} \boldsymbol{u}\right\|_{L^{2}\left(t_{n-1}, t_{n} ; L^{2}\left(\Omega^{\mathrm{f}}\right)\right)}+\tau^{-\frac{1}{2}}\left\|\partial_{t} \boldsymbol{\theta}_{\pi}\right\|_{L^{2}\left(t_{n-1}, t_{n} ; L^{2}\left(\Omega^{\mathrm{f}}\right)\right)}\right)\left\|\boldsymbol{\theta}_{h}^{n}\right\|_{0, \Omega^{\mathrm{f}}} \\
& \leq \frac{\left(\tau \rho^{\mathrm{f}} C_{\mathrm{P}}\right)^{2}}{2 \epsilon_{1} \mu}\left\|\partial_{t t} \boldsymbol{u}\right\|_{L^{2}\left(t_{n-1}, t_{n} ; L^{2}\left(\Omega^{\mathrm{f}}\right)\right)}^{2}+\frac{\left(\rho^{\mathrm{f}} C_{\mathrm{P}}\right)^{2}}{2 \epsilon_{1} \mu} \underbrace{\left\|\partial_{t} \boldsymbol{\theta}_{\pi}\right\|_{L^{2}\left(t_{n-1}, t_{n} ; L^{2}\left(\Omega^{\mathrm{f}}\right)\right)}^{2}}_{\stackrel{\text { def }}{=} T_{1,1}}+\epsilon_{1} \tau\left\|\boldsymbol{\theta}_{h}^{n}\right\|_{\boldsymbol{V}^{2}}^{2} .
\end{aligned}
$$

In this estimate, the last term will be absorbed in the left-hand side of (59) for $\epsilon_{1}>0$ small enough, using Korn's inequality. For the second term, $T_{1,1}$, we use the approximation properties of the fluid velocity projection operator, viz.:

- $\alpha_{1}=0$ : from $(39)_{2}$, we get

$$
T_{1,1} \leq h^{4}\left(c_{\mu}^{2} \mu\left\|\partial_{t} \boldsymbol{u}\right\|_{L^{2}\left(t_{n-1}, t_{n} ; H^{2}\left(\Omega^{\mathrm{f}}\right)\right)}^{2}+\tilde{c}_{\mu}^{2}\left\|\partial_{t} \boldsymbol{u}\right\|_{L^{2}\left(t_{n-1}, t_{n} ; H^{2}(\Sigma)\right)}^{2}\right) ;
$$

- $\alpha_{1}>0$ : we combine the Poincaré inequality with estimate (43), which yields

$$
T_{1,1} \leq C_{\mathrm{P}}^{2}\left\|\partial_{t} \boldsymbol{\theta}_{\pi}\right\|_{L^{2}\left(t_{n-1}, t_{n} ; H^{1}\left(\Omega^{\mathrm{f}}\right)\right)}^{2} \lesssim h^{2} C_{\mathrm{P}}^{2}\left(\left\|\partial_{t} \boldsymbol{u}\right\|_{L^{2}\left(t_{n-1}, t_{n} ; H^{2}\left(\Omega^{\mathrm{f}}\right)\right)}^{2}+\frac{\alpha_{1} \beta_{\mathrm{e}}}{\mu}\left\|\partial_{t} \dot{\boldsymbol{d}}\right\|_{L^{2}\left(t_{n-1}, t_{n} ; H^{2}\left(\Omega^{\mathrm{f}}\right)\right)}^{2}\right) .
$$

For the solid term $T_{2}$, we have

$$
\begin{aligned}
\left|T_{2}\left(\tau \dot{\boldsymbol{\xi}}^{n-\frac{1}{2}}\right)\right| & \leq \rho^{\mathrm{s}} \tau\left(\left\|\left(\partial_{t}-\partial_{\tau}\right) \dot{\boldsymbol{d}}^{n}\right\|_{0, \Omega^{\mathrm{s}}}+\left\|\partial_{\tau} \dot{\boldsymbol{\xi}}_{\pi}^{n}\right\|_{0, \Omega^{\mathrm{s}}}\right)\left\|\dot{\boldsymbol{\xi}}^{n-\frac{1}{2}}\right\|_{0, \Omega^{\mathrm{s}}} \\
& \leq \rho^{\mathrm{s}} \tau\left(\tau^{\frac{1}{2}}\left\|\partial_{t t} \dot{\boldsymbol{d}}\right\|_{L^{2}\left(t_{n-1}, t_{n} ; L^{2}\left(\Omega^{\mathrm{s}}\right)\right)}+\tau^{-\frac{1}{2}}\left\|\partial_{t} \dot{\boldsymbol{\xi}}_{\pi}\right\|_{L^{2}\left(t_{n-1}, t_{n} ; L^{2}\left(\Omega^{\mathrm{s}}\right)\right)}\right)\left\|\dot{\boldsymbol{\xi}}^{n-\frac{1}{2}}\right\|_{0, \Omega^{\mathrm{s}}} \\
& \leq \frac{\rho^{\mathrm{s}} T \tau^{2}}{2 \epsilon_{2}}\left\|\partial_{t t} \dot{\boldsymbol{d}}\right\|_{L^{2}\left(t_{n-1}, t_{n} ; L^{2}\left(\Omega^{\mathrm{s}}\right)\right)}^{2}+\frac{\rho^{\mathrm{s}} T}{2 \epsilon_{2}} \underbrace{\left\|\partial_{t} \dot{\boldsymbol{\xi}}_{\pi}\right\|_{L^{2}\left(t_{n-1}, t_{n} ; L^{2}\left(\Omega^{\mathrm{s}}\right)\right)}^{2}}_{\stackrel{\text { def }}{=} T_{2,1}}+\epsilon_{2} \frac{\rho^{\mathrm{s}} \tau}{T}\left\|\dot{\boldsymbol{\xi}}^{n-\frac{1}{2}}\right\|_{0, \Omega^{\mathrm{s}}}^{2} .
\end{aligned}
$$

$\mathrm{RR} \mathrm{n}^{\circ} 8670$ 
Note that the last term is distributed in the whole solid domain $\Omega^{\mathrm{s}}$ and, hence, it cannot be controlled via the fluid viscous dissipation as in the case of the coupling with a thin-walled solid (see [20, Theorem 3]). As we shall detail below (see estimate (67)), this term can be controlled, for some $\epsilon_{2}>0$ small enough, with the additional dissipation and truncation error introduced by the splitting. For the term $T_{2,1}$ we use the approximation properties of the solid velocity projection operator, namely:

- $\alpha_{1}=0:$ from (38), we get

$$
T_{2,1} \lesssim h^{4}\left\|\partial_{t} \dot{d}\right\|_{L^{2}\left(t_{n-1}, t_{n} ; H^{2}\left(\Omega^{\mathrm{s}}\right)\right)}^{2}
$$

- $\alpha_{1}>0$ : using (43) and the $H^{1}$-coercivity of the elastic bilinear form (30), we get

$$
T_{2,1} \leq C_{\mathrm{P}}^{2}\left\|\partial_{t} \dot{\boldsymbol{\xi}}_{\pi}\right\|_{L^{2}\left(t_{n-1}, t_{n} ; H^{1}\left(\Omega^{\mathrm{s}}\right)\right)}^{2} \lesssim \frac{h^{2} C_{\mathrm{P}}^{2}}{\alpha_{\mathrm{e}}}\left(\frac{\mu}{\alpha_{1}}\left\|\partial_{t} \boldsymbol{u}\right\|_{L^{2}\left(t_{n-1}, t_{n} ; H^{2}\left(\Omega^{\mathrm{f}}\right)\right)}^{2}+\beta_{\mathrm{e}}\left\|\partial_{t} \dot{\boldsymbol{d}}\right\|_{L^{2}\left(t_{n-1}, t_{n} ; H^{2}\left(\Omega^{\mathrm{f}}\right)\right)}^{2}\right) .
$$

The treatment of the terms $T_{3,1}$ and $T_{3,2}$ depends particularly on the nature of the fluid-solid velocity projection operator. We hence treat each case separately:

- $\alpha_{1}=0$ : From (36), (34), (39) and since divu $\boldsymbol{u}^{n}=0$ in $\Omega^{\mathrm{f}}$, we get

$$
\begin{aligned}
\left|\tau T_{3,1}\left(\boldsymbol{\theta}_{h}^{n}, y_{h}^{n}\right)\right| & \leq \tau\left|a\left(\boldsymbol{\theta}_{\pi}^{n}, \boldsymbol{\theta}_{h}^{n}\right)\right|+\tau\left|b\left(y_{\pi}^{n}, \boldsymbol{\theta}_{h}^{n}\right)\right|+\tau\left|s_{h}\left(P_{h} \boldsymbol{u}^{n}, y_{h}^{n}\right)\right|+\tau\left|s_{h}\left(\Pi_{h} p^{n}, y_{h}^{n}\right)\right| \\
& \lesssim \frac{\tau h^{2}}{\varepsilon_{3}}\left(\mu\left\|\boldsymbol{u}^{n}\right\|_{2, \Omega^{\mathrm{f}}}^{2}+\mu^{-1}\left\|p^{n}\right\|_{1, \Omega^{\mathrm{f}}}^{2}\right)+\varepsilon_{3} \tau\left(\left\|\boldsymbol{\theta}_{h}^{n}\right\|_{\boldsymbol{V}^{\mathrm{f}}}^{2}+\left|y_{h}^{n}\right|_{s_{h}}^{2}\right) .
\end{aligned}
$$

On the other hand, since $\alpha_{1}=0$, we have

$$
\left|T_{3,2}\left(\tau \dot{\boldsymbol{\xi}}^{n-\frac{1}{2}}\right)\right|=\tau \alpha_{0} \rho^{\mathrm{s}}\left|\left(\dot{\boldsymbol{\xi}}_{\pi}^{n}, \boldsymbol{\xi}^{n-\frac{1}{2}}\right)_{\Omega^{\mathrm{s}}}\right| \leq \frac{\alpha_{0} \rho^{\mathrm{s}} \tau h^{2}}{4 \epsilon_{4}}\left\|\dot{\boldsymbol{d}}^{n}\right\|_{2, \Omega^{\mathrm{s}}}^{2}+\epsilon_{4} \alpha_{0} \rho^{\mathrm{s}} \tau\left\|\dot{\boldsymbol{\xi}}_{h}^{n-\frac{1}{2}}\right\|_{0, \Omega^{\mathrm{s}}}^{2} ;
$$

- $\alpha_{1}>0$ : from (40) and since divu ${ }^{n}=0$ in $\Omega^{\mathrm{f}}$, we have

$$
\begin{aligned}
& \left|\tau T_{3,1}\left(\boldsymbol{\theta}_{h}^{n}, y_{h}^{n}\right)+\tau T_{3,2}\left(\dot{\boldsymbol{\xi}}^{n-\frac{1}{2}}\right)\right| \leq \tau\left|b\left(P_{h}\left(\boldsymbol{u}^{n}, \dot{\boldsymbol{d}}^{n}\right), \boldsymbol{\theta}_{h}^{n}\right)\right|+\tau\left|b\left(y_{\pi}^{n}, \boldsymbol{\theta}_{h}^{n}\right)\right|+\tau\left|s_{h}\left(\Pi_{h} p^{n}, y_{h}^{n}\right)\right| \\
& +\tau\left|s_{h}\left(P_{h}\left(\boldsymbol{u}^{n}, \dot{\boldsymbol{d}}^{n}\right), y_{h}^{n}\right)\right|+\tau \alpha_{0} \rho^{\mathrm{s}}\left|\left(\dot{\boldsymbol{\xi}}_{\pi}^{n}, \dot{\boldsymbol{\xi}}_{h}^{n-\frac{1}{2}}\right)_{\Omega^{\mathrm{s}}}\right| \\
& \leq \frac{\tau\left(\epsilon_{3}+\epsilon_{4}\right)}{2}\left\|\boldsymbol{\theta}_{h}^{n}\right\|_{\boldsymbol{V}^{\mathrm{f}}}^{2}+\frac{\tau}{2}\left|y_{h}^{n}\right|_{s_{h}}^{2}+\epsilon_{5} \tau \alpha_{0} \rho^{\mathrm{s}}\left\|\dot{\boldsymbol{\xi}}_{h}^{n-\frac{1}{2}}\right\|_{0, \Omega^{\mathrm{s}}}^{2} \\
& +\underbrace{\frac{\tau}{2 \epsilon_{3}}\left\|P_{h}\left(\boldsymbol{u}^{n}, \dot{\boldsymbol{d}}^{n}\right)\right\|_{Q}^{2}+\frac{\tau}{2 \epsilon_{4}}\left\|y_{\pi}^{n}\right\|_{Q}^{2}+\frac{\tau}{2}\left(\left|\Pi_{h} p^{n}\right|_{s_{h}}^{2}+\left|P_{h}\left(\boldsymbol{u}^{n}, \dot{\boldsymbol{d}}^{n}\right)\right|_{s_{h}}^{2}\right)+\frac{\tau \alpha_{0} \rho^{\mathrm{s}}}{4 \epsilon_{5}}\left\|\dot{\boldsymbol{\xi}}_{\pi}^{n}\right\|_{0, \Omega^{\mathrm{s}}}^{2}}_{\stackrel{\text { def }}{=} T_{3,3}} .
\end{aligned}
$$

For $\epsilon_{3}, \epsilon_{4}>0$ small enough, the first part of the right-hand side can be absorbed by the left-hand side of (59) using Korn's inequality. The control of the term $\epsilon_{5} \alpha_{0} \rho^{\mathrm{s}} \tau\left\|\dot{\boldsymbol{\xi}}_{h}^{n-\frac{1}{2}}\right\|_{0, \Omega^{\mathrm{s}}}^{2}$ will be discussed below (see estimate (67)). The remaining terms in the right-hand side of (63) can be estimated via (30), (43) and (34), which yields

$$
\begin{aligned}
T_{3,3} \lesssim \tau h^{2}\left(\mu\left\|\boldsymbol{u}^{n}\right\|_{2, \Omega^{\mathrm{f}}}^{2}+\alpha_{1} \beta_{\mathrm{e}}\left\|\dot{\boldsymbol{d}}^{n}\right\|_{2, \Omega^{\mathrm{s}}}^{2}\right. & \left.+\mu^{-1}\left\|p^{n}\right\|_{1, \Omega^{\mathrm{f}}}^{2}\right) \\
& +\tau h^{2} \frac{\alpha_{0} \rho^{\mathrm{s}} C_{\mathrm{P}}^{2}}{4 \alpha_{\mathrm{e}}}\left(\frac{\mu}{\alpha_{1}}\left\|\boldsymbol{u}^{n}\right\|_{2, \Omega^{\mathrm{f}}}^{2}+\beta_{\mathrm{e}}\left\|\dot{\boldsymbol{d}}^{n}\right\|_{2, \Omega^{\mathrm{s}}}^{2}\right) .
\end{aligned}
$$


We now consider the mass-lumping term $T_{4}$. To this purpose, we first note that, owing to (29) and using a Taylor expansion, we have

$$
\begin{gathered}
\left|T_{4}\left(\tau \dot{\boldsymbol{\xi}}^{n-\frac{1}{2}}\right)\right| \lesssim h \tau \rho^{\mathrm{s}}\left\|\left(\partial_{\tau}+\alpha_{0}\right) \boldsymbol{P}_{h}^{\mathrm{s}}\left(\boldsymbol{u}^{n}, \dot{\boldsymbol{d}}^{n}\right)\right\|_{1, \Omega^{\mathrm{s}}}\left\|\dot{\boldsymbol{\xi}}^{n-\frac{1}{2}}\right\|_{0, \Omega^{\mathrm{s}}} \\
\lesssim \frac{\rho^{\mathrm{s}} T \tau h^{2}}{\epsilon_{6}}\left\|\left(\partial_{\tau}+\alpha_{0}\right) \boldsymbol{P}_{h}^{\mathrm{s}}\left(\boldsymbol{u}^{n}, \dot{\boldsymbol{d}}^{n}\right)\right\|_{1, \Omega^{\mathrm{s}}}^{2}+\frac{\epsilon_{6} \rho^{\mathrm{s}} \tau}{T}\left\|\dot{\boldsymbol{\xi}}^{n-\frac{1}{2}}\right\|_{0, \Omega^{\mathrm{s}}}^{2} \\
\underbrace{\frac{\rho^{\mathrm{s}} T \tau h^{2}}{\epsilon_{6}}\left(\tau\left\|\boldsymbol{P}_{h}^{\mathrm{s}}\left(\partial_{t t} \boldsymbol{u}, \partial_{t t} \dot{\boldsymbol{d}}\right)\right\|_{L^{2}\left(t_{n-1}, t_{n} ; H^{1}\left(\Omega^{\mathrm{s}}\right)\right)}^{2}+\left\|\boldsymbol{P}_{h}^{\mathrm{s}}\left(\partial_{t} \boldsymbol{u}^{n}, \partial_{t} \dot{\boldsymbol{d}}^{n}\right)\right\|_{1, \Omega^{\mathrm{s}}}^{2}+\alpha_{0}^{2}\left\|\boldsymbol{P}_{h}^{\mathrm{s}}\left(\boldsymbol{u}^{n}, \dot{\boldsymbol{d}}^{n}\right)\right\|_{1, \Omega^{\mathrm{s}}}^{2}\right)}_{\underbrace{=}_{\text {def }} T_{4,1}}
\end{gathered}
$$

As above, the last term will be controlled for $\epsilon_{6}>0$ small enough through the numerical dissipation provided by the splitting (see estimate (67)). The term $T_{4,1}$ can be controlled via (38) or (30) and (42) depending of the type of solid velocity projection operator. Thus,

$$
\left\|\boldsymbol{P}_{h}^{\mathrm{s}}\left(\boldsymbol{u}^{n}, \dot{\boldsymbol{d}}^{n}\right)\right\|_{1, \Omega^{\mathrm{s}}}^{2} \lesssim \begin{cases}h^{2}\left\|\dot{\boldsymbol{d}}^{n}\right\|_{2, \Omega^{\mathrm{s}}}^{2}+\left\|\dot{\boldsymbol{d}}^{n}\right\|_{1, \Omega^{\mathrm{s}}}^{2} & \text { if } \alpha_{1}=0, \\ \frac{1}{\alpha_{\mathrm{e}}}\left(\frac{\mu}{\alpha_{1}}\left\|\boldsymbol{u}^{n}\right\|_{1, \Omega^{\mathrm{f}}}^{2}+\beta_{\mathrm{e}}\left\|\dot{\boldsymbol{d}}^{n}\right\|_{1, \Omega^{\mathrm{s}}}^{2}\right) & \text { if } \quad \alpha_{1}>0\end{cases}
$$

and similarly for the other contributions in $T_{4,1}$.

For the term $T_{7}$, using the continuity of the bilinear form $a^{\mathrm{e}}$, we have

$$
\left|T_{7}\right| \leq \tau\left\|\boldsymbol{\xi}_{h}^{n}\right\|_{\mathrm{e}}\left\|\boldsymbol{z}_{h}^{n}\right\|_{\mathrm{e}} \leq \frac{\tau}{2 T}\left\|\boldsymbol{\xi}_{h}^{n}\right\|_{\mathrm{e}}^{2}+\frac{T \tau}{2}\left\|\boldsymbol{z}_{h}^{n}\right\|_{\mathrm{e}}^{2}
$$

where the first term of the left-hand side can be treated by applying Gronwall's Lemma to (59) and the last one is controlled by the approximation result (51).

We now recall that the estimates (62)-(64) assume that we have a control on

$$
\epsilon_{7} \underbrace{\left(\alpha_{0}+\frac{1}{T}\right)}_{\stackrel{\text { def }}{=} c_{\alpha_{0}, T}} \tau \rho^{\mathrm{s}}\left\|\dot{\boldsymbol{\xi}}^{n-\frac{1}{2}}\right\|_{0, \Omega^{\mathrm{s}}}^{2}
$$

with $\epsilon_{7} \stackrel{\text { def }}{=} \max \left\{\epsilon_{2}, \epsilon_{4}, \epsilon_{5}, \epsilon_{6}\right\}$ small enough. To this purpose we note that, owing to (48) and (54), we have

$$
\begin{gathered}
\epsilon_{7} c_{\alpha_{0}, T} \tau \rho^{\mathrm{s}}\left\|\dot{\boldsymbol{\xi}}^{n-\frac{1}{2}}\right\|_{0, \Omega^{\mathrm{s}}}^{2} \lesssim \epsilon_{7} c_{\alpha_{0}, T} \tau \rho^{\mathrm{s}}\left\|\dot{\boldsymbol{\xi}}_{h}^{n}\right\|_{0, \Omega^{\mathrm{s}}}^{2}+\underbrace{\frac{\epsilon_{7} \tau^{2}}{\rho^{\mathrm{s}}}\left\|\boldsymbol{L}_{h}^{\mathrm{e}}\left(\boldsymbol{\xi}_{h}^{n}-\boldsymbol{\xi}_{h}^{n, \star}\right)+\boldsymbol{L}_{h}^{\mathrm{v}}\left(\dot{\boldsymbol{\xi}}_{h}^{n}-\dot{\boldsymbol{\xi}}_{h}^{n, \star}\right)\right\|_{0, \Omega^{\mathrm{s}}}^{2}}_{\stackrel{\text { def }}{=} T_{8}} \\
+\underbrace{\frac{\epsilon_{7} \tau^{2}}{\rho^{\mathrm{s}}}\left\|\boldsymbol{L}_{h}^{\mathrm{e}}\left(\boldsymbol{d}^{n}-\boldsymbol{d}^{n, \star}\right)+\boldsymbol{L}_{h}^{\mathrm{v}} \boldsymbol{P}_{h}^{\mathrm{s}}\left(\boldsymbol{u}^{n}-\boldsymbol{u}^{n, \star}, \dot{\boldsymbol{d}}^{n}-\dot{\boldsymbol{d}}^{n, \star}\right)\right\|_{0, \Omega^{\mathrm{s}}}^{2}}_{\stackrel{\text { def }}{=} T_{9}} \cdot
\end{gathered}
$$

The first part term will be left in the right-hand side and will be treated via Gronwall's Lemma. The terms $T_{8}$ and $T_{9}$ will, respectively, be handled in terms of $r \in\{0,1,2\}$ via the numerical dissipation provided by the fluid-solid splitting (term $T_{5,2}$ as shown below) and a Taylor expansion.

$\mathrm{RR} \mathrm{n}^{\circ} 8670$ 
We now estimate the remaining terms given by (60) for each choice of the extrapolation order $r$. The bounds for $T_{5,1}+T_{5,2}$ basically follow from the arguments of the proof of Theorem 2 reported in [21, Section 4.2], we recall them for the sake of completeness.

Case $r=0$. We have

$$
T_{5,1}+T_{5,2} \geq-\frac{\rho^{\mathrm{s}}}{3}\left\|\dot{\boldsymbol{\xi}}_{h}^{n}-\dot{\boldsymbol{\xi}}_{h}^{n-1}\right\|_{*}^{2}+\frac{\tau^{2}}{4 \rho^{\mathrm{s}}}\left\|\boldsymbol{L}_{h}^{\mathrm{e}} \boldsymbol{\xi}_{h}^{n}+\boldsymbol{L}_{h}^{\mathrm{v}} \dot{\boldsymbol{\xi}}_{h}^{n}\right\|_{*}^{2}
$$

The first term can be absorbed in (59) by the numerical dissipation of the time-stepping in the solid. The additional dissipation provided by (68) guarantees the control of the term $T_{8}$, for $\epsilon_{7}>0$ small enough. The term $T_{6}$ is estimated as follows:

$$
T_{6,1}+T_{6,2} \lesssim \frac{\rho^{\mathrm{s}}}{6}\left\|\dot{\boldsymbol{\xi}}_{h}^{n}-\dot{\boldsymbol{\xi}}_{h}^{n-1}\right\|_{*}^{2}+\frac{\tau^{2}}{8 \rho^{\mathrm{s}}}\left\|\boldsymbol{L}_{h}^{\mathrm{e}} \boldsymbol{\xi}_{h}^{n}+\boldsymbol{L}_{h}^{\mathrm{v}} \dot{\boldsymbol{\xi}}_{h}^{n}\right\|_{*}^{2}+\frac{7 \tau^{2}}{2 \rho^{\mathrm{s}}} \underbrace{\left\|\boldsymbol{L}_{h}^{\mathrm{e}} \boldsymbol{d}^{n}+\boldsymbol{L}_{h}^{\mathrm{v}} \boldsymbol{P}_{h}^{\mathrm{s}}\left(\boldsymbol{u}^{n}, \dot{\boldsymbol{d}}^{n}\right)\right\|_{*}^{2}}_{\stackrel{\text { def }}{=} T_{6,3}} .
$$

Moreover, using a Taylor expansion, for the last term we have

$$
T_{6,3} \lesssim \tau^{-1}\left\|\boldsymbol{L}_{h}^{\mathrm{e}} \boldsymbol{d}+\boldsymbol{L}_{h}^{\mathrm{v}} \boldsymbol{P}_{h}^{\mathrm{s}}(\boldsymbol{u}, \dot{\boldsymbol{d}})\right\|_{L^{2}\left(t_{n-1}, t_{n} ; L^{2}\left(\Omega^{\mathrm{s}}\right)\right)}^{2}+\tau\left\|\partial_{t}\left(\boldsymbol{L}_{h}^{\mathrm{e}} \boldsymbol{d}+\boldsymbol{L}_{h}^{\mathrm{v}} \boldsymbol{P}_{h}^{\mathrm{s}}(\boldsymbol{u}, \dot{\boldsymbol{d}})\right)\right\|_{L^{2}\left(t_{n-1}, t_{n} ; L^{2}\left(\Omega^{\mathrm{s}}\right)\right)}^{2},
$$

which provides also a bound for $T_{9}$.

Finally, estimate (56) follows by inserting (61)-(65) and (68)-(69) in (59), summing over $m=1, \ldots, n$ and applying Gronwall's Lemma and Korn's inequality.

Case $r=1$. Using the modified velocity-displacement relation (49), we have

$$
\begin{aligned}
T_{5,1}+T_{5,2}= & \frac{\tau^{2}}{2}\left(\left\|\dot{\boldsymbol{\xi}}_{h}^{n}\right\|_{\mathrm{e}}^{2}-\left\|\dot{\boldsymbol{\xi}}_{h}^{n-1}\right\|_{\mathrm{e}}^{2}+\left\|\dot{\boldsymbol{\xi}}_{h}^{n}-\dot{\boldsymbol{\xi}}_{h}^{n-1}\right\|_{\mathrm{e}}^{2}\right)+\tau\left\|\dot{\boldsymbol{\xi}}_{h}^{n}-\dot{\boldsymbol{\xi}}_{h}^{n-1}\right\|_{\mathrm{v}}^{2} \\
& +\frac{\tau^{2}}{2 \rho^{\mathrm{s}}}\left(\left\|\boldsymbol{L}_{h}^{\mathrm{e}} \boldsymbol{\xi}_{h}^{n}+\boldsymbol{L}_{h}^{\mathrm{v}} \dot{\boldsymbol{\xi}}_{h}^{n}\right\|_{*}^{2}-\left\|\boldsymbol{L}_{h}^{\mathrm{e}} \boldsymbol{\xi}_{h}^{n-1}+\boldsymbol{L}_{h}^{\mathrm{v}} \dot{\boldsymbol{\xi}}_{h}^{n-1}\right\|_{*}^{2}\right) \\
& +\frac{\tau^{2}}{2 \rho^{\mathrm{s}}}\left\|\boldsymbol{L}_{h}^{\mathrm{e}}\left(\boldsymbol{\xi}_{h}^{n}-\boldsymbol{\xi}_{h}^{n-1}\right)+\boldsymbol{L}_{h}^{\mathrm{v}}\left(\dot{\boldsymbol{\xi}}_{h}^{n}-\dot{\boldsymbol{\xi}}_{h}^{n-1}\right)\right\|_{*}^{2}-\underbrace{\tau^{2} a^{\mathrm{e}}\left(\dot{\boldsymbol{\xi}}_{h}^{n}-\dot{\boldsymbol{\xi}}_{h}^{n-1}, \boldsymbol{z}_{h}^{n}\right)}_{T_{5,3}},
\end{aligned}
$$

where

$$
\left|T_{5,3}\right| \leq \frac{\tau^{2}}{4}\left\|\dot{\boldsymbol{\xi}}_{h}^{n}-\dot{\boldsymbol{\xi}}_{h}^{n-1}\right\|_{\mathrm{e}}^{2}+\tau^{2}\left\|\boldsymbol{z}_{h}^{n}\right\|_{\mathrm{e}}^{2}
$$

Hence, this term can be controlled with the extra dissipation provided by (70) and the estimate (51). Moreover, the additional dissipation provided by the last line of (70) guarantees the control of the term $T_{8}$, for $\epsilon_{7}>0$ sufficiently small. On the other hand, we have

$$
\begin{aligned}
T_{6,1}+T_{6,2} \leq \frac{\rho^{\mathrm{s}}}{4}\left\|\dot{\boldsymbol{\xi}}_{h}^{n}-\dot{\boldsymbol{\xi}}_{h}^{n-1}\right\|_{*}^{2}+ & \frac{\tau^{3}}{2 T \rho^{\mathrm{s}}}\left\|\boldsymbol{L}_{h}^{\mathrm{e}} \boldsymbol{\xi}_{h}^{n}+\boldsymbol{L}_{h}^{\mathrm{v}} \dot{\boldsymbol{\xi}}_{h}^{n}\right\|_{*}^{2} \\
& +\frac{\tau^{2}(T+\tau)}{2 \rho^{\mathrm{s}}}\left\|\partial_{t}\left(\boldsymbol{L}_{h}^{\mathrm{e}} \boldsymbol{d}+\boldsymbol{L}_{h}^{\mathrm{v}} \boldsymbol{P}_{h}^{\mathrm{s}}(\boldsymbol{u}, \dot{\boldsymbol{d}})\right)\right\|_{L^{2}\left(t_{n-1}, t_{n} ; L^{2}\left(\Omega^{\mathrm{s}}\right)\right)}^{2}
\end{aligned}
$$

The first term will be absorbed by the left-hand side of (59) while the second term will be controlled with (70) and Gronwall's Lemma. At last, for the term $T_{9}$, using again a Taylor expansion we have

$$
T_{9} \leq \epsilon_{7} \frac{\tau^{3}}{\rho^{\mathrm{s}}}\left\|\partial_{t}\left(\boldsymbol{L}_{h}^{\mathrm{e}} \boldsymbol{d}+\boldsymbol{L}_{h}^{\mathrm{v}} \boldsymbol{P}_{h}^{\mathrm{s}}(\boldsymbol{u}, \dot{\boldsymbol{d}})\right)\right\|_{L^{2}\left(t_{n-1}, t_{n} ; L^{2}\left(\Omega^{\mathrm{s}}\right)\right)}^{2} .
$$


Finally, we conclude by inserting (61)-(65) and (70)-(71) into (59), by summing over $m=$ $2, \ldots, n$ and applying the discrete Gronwall's Lemma and Korn's inequality. The right-hand side contributions at time $t_{1}$ coming from the initialization step are controlled by using (56) with $r=0, T=\tau$ and $n=1$.

Case $r=2$. The modified velocity-displacement relation (49) yields

$$
\begin{aligned}
T_{5,1}= & \tau^{2}\left\|\dot{\boldsymbol{\xi}}_{h}^{n}-\dot{\boldsymbol{\xi}}_{h}^{n-1}\right\|_{\mathrm{e}}^{2}+\frac{\tau}{2}\left(\left\|\dot{\boldsymbol{\xi}}_{h}^{n}-\dot{\boldsymbol{\xi}}_{h}^{n-1}\right\|_{\mathrm{v}, *}^{2}-\left\|\dot{\boldsymbol{\xi}}_{h}^{n-1}-\dot{\boldsymbol{\xi}}_{h}^{n-2}\right\|_{\mathrm{v}, *}^{2}+\left\|\dot{\boldsymbol{\xi}}_{h}^{n}-2 \dot{\boldsymbol{\xi}}_{h}^{n-1}+\dot{\boldsymbol{\xi}}_{h}^{n-2}\right\|_{\mathrm{v}, *}^{2}\right) \\
& \underbrace{-\tau^{2} a^{\mathrm{e}}\left(\dot{\boldsymbol{\xi}}_{h}^{n}-\dot{\boldsymbol{\xi}}_{h}^{n-1}, \boldsymbol{z}_{h}^{n}-\boldsymbol{z}_{h}^{n-1}\right)}_{\stackrel{\text { def }}{=} T_{5,1,1}},
\end{aligned}
$$

where,

$$
\left|T_{5,1,1}\right| \leq \tau^{2}\left\|\dot{\boldsymbol{\xi}}_{h}^{n}-\dot{\boldsymbol{\xi}}_{h}^{n-1}\right\|_{\mathrm{e}}\left\|\boldsymbol{z}_{h}^{n}-\boldsymbol{z}_{h}^{n-1}\right\|_{\mathrm{e}} \leq \frac{\tau^{2}}{4}\left\|\dot{\boldsymbol{\xi}}_{h}^{n}-\dot{\boldsymbol{\xi}}_{h}^{n-1}\right\|_{\mathrm{e}}^{2}+\tau^{2}\left\|\boldsymbol{z}_{h}^{n}-\boldsymbol{z}_{h}^{n-1}\right\|_{\mathrm{e}}^{2}
$$

Here, the first term is controlled by the numerical dissipation provided by (72) while for the last we apply the bound (51) stated in Lemma 5.

The estimation of the term $T_{5,2}$ is more delicate. It is convenient to split it as follows:

$$
\begin{aligned}
T_{5,2}= & \underbrace{\frac{\tau^{2}}{\rho^{\mathbf{s}}}\left(\boldsymbol{L}_{h}^{\mathrm{v}} \dot{\boldsymbol{\xi}}_{h}^{n}, \boldsymbol{L}_{h}^{\mathrm{e}}\left(\boldsymbol{\xi}_{h}^{n}-2 \boldsymbol{\xi}_{h}^{n-1}+\boldsymbol{\xi}_{h}^{n-2}\right)\right)_{*}}_{\stackrel{\text { def }}{=} T_{5,2,1}}+\underbrace{\frac{\tau^{2}}{\rho^{\mathrm{s}}}\left(\boldsymbol{L}_{h}^{\mathrm{e}} \boldsymbol{\xi}_{h}^{n}, \boldsymbol{L}_{h}^{\mathrm{e}}\left(\boldsymbol{\xi}_{h}^{n}-2 \boldsymbol{\xi}_{h}^{n-1}+\boldsymbol{\xi}_{h}^{n-2}\right)\right)_{*}}_{\stackrel{\text { def }}{=} T_{5,2,2}} \\
& +\underbrace{\frac{\tau^{2}}{\rho^{\mathrm{s} \epsilon}}\left(\boldsymbol{L}_{h}^{\mathrm{e}} \boldsymbol{\xi}_{h}^{n}+\boldsymbol{L}_{h}^{\mathrm{v}} \dot{\boldsymbol{\xi}}_{h}^{n}, \boldsymbol{L}_{h}^{\mathrm{v}}\left(\dot{\boldsymbol{\xi}}_{h}^{n}-2 \dot{\boldsymbol{\xi}}_{h}^{n-1}+\dot{\boldsymbol{\xi}}_{h}^{n-2}\right)\right)_{*}}_{\stackrel{\text { def }}{=} T_{5,2,3}} .
\end{aligned}
$$

For the first term, using (7), (8), (49), we have

$$
\begin{aligned}
T_{5,2,1}= & \frac{\tau^{3}}{\rho^{\mathrm{s}}}\left(\boldsymbol{L}_{h}^{\mathrm{v}} \dot{\boldsymbol{\xi}}_{h}^{n}, \boldsymbol{L}_{h}^{\mathrm{e}}\left(\dot{\boldsymbol{\xi}}_{h}^{n}-\dot{\boldsymbol{\xi}}_{h}^{n-1}\right)\right)_{*} \underbrace{-\frac{\tau^{3}}{\rho^{\mathrm{s}}}\left(\boldsymbol{L}_{h}^{\mathrm{v}} \dot{\boldsymbol{\xi}}_{h}^{n}, \boldsymbol{L}_{h}^{\mathrm{e}}\left(\boldsymbol{z}_{h}^{n}-\boldsymbol{z}_{h}^{n-1}\right)\right)_{*}}_{\stackrel{\text { def }}{=} T_{5,2,1,1}} \\
= & \frac{\alpha_{0} \tau^{3}}{2}\left(\left\|\dot{\boldsymbol{\xi}}_{h}^{n}\right\|_{\mathrm{e}}^{2}-\left\|\dot{\boldsymbol{\xi}}_{h}^{n-1}\right\|_{\mathrm{e}}^{2}+\left\|\dot{\boldsymbol{\xi}}_{h}^{n}-\dot{\boldsymbol{\xi}}_{h}^{n-1}\right\|_{\mathrm{e}}^{2}\right) \\
& +\frac{\alpha_{1} \tau^{3}}{2 \rho^{\mathrm{s}}}\left(\left\|\boldsymbol{L}_{h}^{\mathrm{e}} \dot{\boldsymbol{\xi}}_{h}^{n}\right\|_{*}^{2}-\left\|\boldsymbol{L}_{h}^{\mathrm{e}} \dot{\boldsymbol{\xi}}_{h}^{n-1}\right\|_{*}^{2}+\left\|\boldsymbol{L}_{h}^{\mathrm{e}}\left(\dot{\boldsymbol{\xi}}_{h}^{n}-\dot{\boldsymbol{\xi}}_{h}^{n-1}\right)\right\|_{*}^{2}\right)+T_{5,2,1,1} .
\end{aligned}
$$

Moreover, owing to (31), the notation $\omega_{\mathrm{e}} \stackrel{\text { def }}{=} C_{\mathrm{inv}} \sqrt{\beta_{\mathrm{e}} / \rho^{\mathrm{s}}}$ and the stability condition (24), we get

$$
\left|T_{5,2,1,1}\right| \lesssim \frac{\tau^{5} \omega_{\mathrm{e}}^{2}}{T h^{2}}\left(\alpha_{0}+\frac{\alpha_{1} \omega_{\mathrm{e}}^{2}}{h^{2}}\right)\left\|\dot{\boldsymbol{\xi}}_{h}^{n}\right\|_{\mathrm{v}}^{2}+\frac{\tau T}{4}\left\|\boldsymbol{z}_{h}^{n}-\boldsymbol{z}_{h}^{n-1}\right\|_{\mathrm{e}}^{2} \leq \frac{\gamma \tau^{3}}{T}\left\|\dot{\boldsymbol{\xi}}_{h}^{n}\right\|_{\mathrm{v}}^{2}+\frac{\tau T}{4}\left\|\boldsymbol{z}_{h}^{n}-\boldsymbol{z}_{h}^{n-1}\right\|_{\mathrm{e}}^{2}
$$

For the second term of (74) we proceed in a similar fashion, using (49), (31) and the stability $\mathrm{RR} \mathrm{n}^{\circ} 8670$ 
condition (24), which yields

$$
\begin{aligned}
\left|T_{5,2,2}\right| & \leq \frac{\tau^{3}}{\rho^{\mathrm{s}}}\left\|\boldsymbol{L}_{h}^{\mathrm{e}} \boldsymbol{\xi}_{h}^{n}\right\|_{\mathrm{e}}\left(\left\|\dot{\boldsymbol{\xi}}_{h}^{n}-\dot{\boldsymbol{\xi}}_{h}^{n-1}\right\|_{\mathrm{e}}+\left\|\boldsymbol{z}_{h}^{n}-\boldsymbol{z}_{h}^{n-1}\right\|_{\mathrm{e}}\right) \\
& \leq \tau^{6} \frac{\omega_{\mathrm{e}}^{6}}{h^{6}}\left\|\boldsymbol{\xi}_{h}^{n}\right\|_{\mathrm{e}}^{2}+\frac{\rho^{\mathrm{s}}}{2}\left\|\dot{\boldsymbol{\xi}}_{h}^{n}-\dot{\boldsymbol{\xi}}_{h}^{n-1}\right\|_{0, \Omega^{\mathrm{s}}}^{2}+\frac{\rho^{\mathrm{s}}}{2}\left\|\boldsymbol{z}_{h}^{n}-\boldsymbol{z}_{h}^{n-1}\right\|_{0, \Omega^{\mathrm{s}}}^{2} \\
& \leq \gamma \tau\left\|\boldsymbol{\xi}_{h}^{n}\right\|_{\mathrm{e}}^{2}+\frac{\rho^{\mathrm{s}}}{2}\left\|\dot{\boldsymbol{\xi}}_{h}^{n}-\dot{\boldsymbol{\xi}}_{h}^{n-1}\right\|_{0, \Omega^{\mathrm{s}}}^{2}+\frac{\rho^{\mathrm{s}}}{2}\left\|\boldsymbol{z}_{h}^{n}-\boldsymbol{z}_{h}^{n-1}\right\|_{0, \Omega^{\mathrm{s}}}^{2} .
\end{aligned}
$$

At last, owing to (7), (31) and the stability condition (24), we have

$$
\begin{aligned}
\left|T_{5,2,3}\right| & \lesssim \frac{\tau^{2}}{\rho^{\mathrm{s}}}\left\|\boldsymbol{L}_{h}^{\mathrm{e}} \boldsymbol{\xi}_{h}^{n}+\boldsymbol{L}_{h}^{\mathrm{v}} \dot{\boldsymbol{\xi}}_{h}^{n}\right\|_{\mathrm{v}}\left\|\dot{\boldsymbol{\xi}}_{h}^{n}-2 \dot{\boldsymbol{\xi}}_{h}^{n-1}+\dot{\boldsymbol{\xi}}_{h}^{n-2}\right\|_{\mathrm{v}} \\
& \lesssim \frac{2 \tau^{3}}{\left(\rho^{\mathrm{s}}\right)^{2}}\left\|\boldsymbol{L}_{h}^{\mathrm{e}} \boldsymbol{\xi}_{h}^{n}\right\|_{\mathrm{v}}^{2}+\frac{2 \tau^{3}}{\left(\rho^{\mathrm{s}}\right)^{2}}\left\|\boldsymbol{L}_{h}^{\mathrm{v}} \dot{\boldsymbol{\xi}}_{h}^{n}\right\|_{\mathrm{v}}^{2}+\frac{\tau}{4}\left\|\dot{\boldsymbol{\xi}}_{h}^{n}-2 \dot{\boldsymbol{\xi}}_{h}^{n-1}+\dot{\boldsymbol{\xi}}_{h}^{n-2}\right\|_{\mathrm{v}}^{2} \\
& \lesssim 2 \frac{\tau^{3} \omega_{\mathrm{e}}^{2}}{h^{2}}\left(\alpha_{0}+\frac{\alpha_{1} \omega_{\mathrm{e}}^{2}}{h^{2}}\right)\left\|\boldsymbol{\xi}_{h}^{n}\right\|_{\mathrm{e}}^{2}+2 \tau^{3}\left(\alpha_{0}+\frac{\alpha_{1} \omega_{\mathrm{e}}^{2}}{h^{2}}\right)^{2}\left\|\dot{\boldsymbol{\xi}}_{h}^{n}\right\|_{\mathrm{v}}^{2}+\frac{\tau}{4}\left\|\dot{\boldsymbol{\xi}}_{h}^{n}-2 \dot{\boldsymbol{\xi}}_{h}^{n-1}+\dot{\boldsymbol{\xi}}_{h}^{n-2}\right\|_{\mathrm{v}}^{2} \\
& \lesssim 2 \gamma \tau\left\|\boldsymbol{\xi}_{h}^{n}\right\|_{\mathrm{e}}^{2}+2 \tau \delta^{2}\left\|\dot{\boldsymbol{\xi}}_{h}^{n}\right\|_{\mathrm{v}}^{2}+\frac{\tau}{4}\left\|\dot{\boldsymbol{\xi}}_{h}^{n}-2 \dot{\boldsymbol{\xi}}_{h}^{n-1}+\dot{\boldsymbol{\xi}}_{h}^{n-2}\right\|_{\mathrm{v}}^{2}
\end{aligned}
$$

In summary, by inserting the above estimations into (74), we have that

$$
\begin{array}{r}
T_{5,2} \gtrsim \frac{\alpha_{0} \tau^{3}}{2}\left(\left\|\dot{\boldsymbol{\xi}}_{h}^{n}\right\|_{\mathrm{e}}^{2}-\left\|\dot{\boldsymbol{\xi}}_{h}^{n-1}\right\|_{\mathrm{e}}^{2}+\tau^{2}\left\|\partial_{\tau} \dot{\boldsymbol{\xi}}_{h}^{n}\right\|_{\mathrm{e}}^{2}\right)+\frac{\alpha_{1} \tau^{3}}{2 \rho^{\mathrm{s}}}\left(\left\|\boldsymbol{L}_{h}^{\mathrm{e}} \dot{\boldsymbol{\xi}}_{h}^{n}\right\|_{*}^{2}-\left\|\boldsymbol{L}_{h}^{\mathrm{e}} \dot{\boldsymbol{\xi}}_{h}^{n-1}\right\|_{*}^{2}+\tau^{2}\left\|\boldsymbol{L}_{h}^{\mathrm{e}} \partial_{\tau} \dot{\boldsymbol{\xi}}_{h}^{n}\right\|_{*}^{2}\right) \\
-\frac{\tau}{4}\left\|\dot{\boldsymbol{\xi}}_{h}^{n}-2 \dot{\boldsymbol{\xi}}_{h}^{n-1}+\dot{\boldsymbol{\xi}}_{h}^{n-2}\right\|_{\mathrm{v}}^{2}-3 \gamma \tau\left\|\boldsymbol{\xi}_{h}^{n}\right\|_{\mathrm{e}}^{2}-\tau\left(\frac{\gamma \tau^{2}}{T}+2 \delta^{2}\right)\left\|\dot{\boldsymbol{\xi}}_{h}^{n}\right\|_{\mathrm{v}}^{2}-\frac{\rho^{\mathrm{s}}}{2}\left\|\dot{\boldsymbol{\xi}}_{h}^{n}-\dot{\boldsymbol{\xi}}_{h}^{n-1}\right\|_{0, \Omega^{\mathrm{s}}}^{2} \\
-\frac{\rho^{\mathrm{s}}}{2}\left\|\boldsymbol{z}_{h}^{n}-\boldsymbol{z}_{h}^{n-1}\right\|_{0, \Omega^{\mathrm{s}}}^{2}-\frac{\tau T}{4}\left\|\boldsymbol{z}_{h}^{n}-\boldsymbol{z}_{h}^{n-1}\right\|_{\mathrm{e}}^{2}
\end{array}
$$

In the second line, the first term is absorbed into the numerical dissipation provided by (72), the second term is controlled via Gronwall's Lemma, the third by the solid physical dissipation of (59), under the relation (55), and the fourth term by the numerical dissipation of the solid timestepping in (59). The last two terms are estimated with the bound (51) provided by Lemma 5.

We now detail how the term $T_{8}$ is controlled in this case. Using (49), (31) and the stability condition (24), we have

$$
\begin{aligned}
T_{8} & \leq 2 \frac{\epsilon_{7} \tau^{4}}{\rho^{\mathrm{s}}}\left\|\boldsymbol{L}_{h}^{\mathrm{e}}\left(\dot{\boldsymbol{\xi}}_{h}^{n}-\dot{\boldsymbol{\xi}}_{h}^{n-1}\right)+\boldsymbol{L}_{h}^{\mathrm{e}}\left(\boldsymbol{z}_{h}^{n}-\boldsymbol{z}_{h}^{n-1}\right)\right\|_{0, \Omega^{\mathrm{s}}}^{2}+2 \frac{\epsilon_{7} \tau^{2}}{\rho^{\mathrm{s}}}\left\|\boldsymbol{L}_{h}^{\mathrm{v}}\left(\dot{\boldsymbol{\xi}}_{h}^{n}-2 \dot{\boldsymbol{\xi}}_{h}^{n-1}+\dot{\boldsymbol{\xi}}_{h}^{n-2}\right)\right\|_{0, \Omega^{\mathrm{s}}}^{2} \\
& \leq 4 \frac{\epsilon_{7} \tau^{4} \omega_{\mathrm{e}}^{2}}{h^{2}}\left(\left\|\dot{\boldsymbol{\xi}}_{h}^{n}-\dot{\boldsymbol{\xi}}_{h}^{n-1}\right\|_{\mathrm{e}}^{2}+\left\|\boldsymbol{z}_{h}^{n}-\boldsymbol{z}_{h}^{n-1}\right\|_{\mathrm{e}}^{2}\right)+2 \epsilon_{7} \tau^{2}\left(\alpha_{0}+\frac{\alpha_{1} \omega_{\mathrm{e}}^{2}}{h^{2}}\right)\left\|\left(\dot{\boldsymbol{\xi}}_{h}^{n}-2 \dot{\boldsymbol{\xi}}_{h}^{n-1}+\dot{\boldsymbol{\xi}}_{h}^{n-2}\right)\right\|_{\mathrm{v}}^{2} \\
& \leq 4 \epsilon_{7}(\gamma \tau)^{\frac{1}{3}} \tau^{2}\left(\left\|\dot{\boldsymbol{\xi}}_{h}^{n}-\dot{\boldsymbol{\xi}}_{h}^{n-1}\right\|_{\mathrm{e}}^{2}+\left\|\boldsymbol{z}_{h}^{n}-\boldsymbol{z}_{h}^{n-1}\right\|_{\mathrm{e}}^{2}\right)+2 \epsilon_{7} \tau^{2} \delta\left\|\left(\dot{\boldsymbol{\xi}}_{h}^{n}-2 \dot{\boldsymbol{\xi}}_{h}^{n-1}+\dot{\boldsymbol{\xi}}_{h}^{n-2}\right)\right\|_{\mathrm{v}}^{2}
\end{aligned}
$$

The first and the last term are, respectively, absorbed into (59) and $T_{5,1}$, for $\epsilon_{7}>0$ small enough. The second term involving $\left\|\boldsymbol{z}_{h}^{n}-\boldsymbol{z}_{h}^{n-1}\right\|_{\mathrm{e}}^{2}$ is controlled via (51).

For the remaining terms $T_{6,1}$ and $T_{6,2}$, we proceed similarly by combining (31) and (24) with 
a Taylor expansion, which yields

$$
\begin{aligned}
\left|T_{6,1}\right|+\left|T_{6,2}\right| \lesssim \frac{\rho^{\mathrm{s}}}{4}\left\|\dot{\boldsymbol{\xi}}_{h}^{n}-\dot{\boldsymbol{\xi}}_{h}^{n-1}\right\|_{*}^{2} & +\frac{(\gamma \tau)^{\frac{1}{3}} \tau}{4 T}\left\|\boldsymbol{\xi}_{h}^{n}\right\|_{\mathrm{e}}^{2}+\frac{\tau^{2} \delta}{4 T}\left\|\dot{\boldsymbol{\xi}}_{h}^{n}\right\|_{\mathrm{v}}^{2} \\
& +\frac{\tau^{4}(\tau+T)}{\rho^{\mathrm{s}}}\left\|\partial_{t t}\left(\boldsymbol{L}_{h}^{\mathrm{e}} \boldsymbol{\xi}+\boldsymbol{L}_{h}^{\mathrm{v}} \boldsymbol{P}_{h}^{\mathrm{s}}(\boldsymbol{u}, \dot{\boldsymbol{d}})\right)\right\|_{L^{2}\left(t_{n-1}, t_{n} ; L^{2}\left(\Omega^{\mathrm{s}}\right)\right)}^{2},
\end{aligned}
$$

where the first three terms can be absorbed into the left-hand side of (59) and using Gronwall's Lemma. At last, for the term $T_{9}$, using the same Taylor expansion, we have

$$
T_{9} \leq \epsilon_{7} \frac{\tau^{5}}{\rho^{\mathrm{s}}}\left\|\partial_{t t}\left(\boldsymbol{L}_{h}^{\mathrm{e}} \boldsymbol{d}+\boldsymbol{L}_{h}^{\mathrm{v}} \boldsymbol{P}_{h}^{\mathrm{s}}(\boldsymbol{u}, \dot{\boldsymbol{d}})\right)\right\|_{L^{2}\left(t_{n-1}, t_{n} ; L^{2}\left(\Omega^{\mathrm{s}}\right)\right)}^{2} .
$$

Finally, estimate (56) follows by inserting (61)-(65) and (72)-(75) in (59), summing over $m=$ $3, \ldots, n$ and applying Gronwall's Lemma. The right-hand side contributions at time $t_{2}$ coming from the initialization step can be controlled by using (56) with $r=1, T=2 \tau$ and $n=2$.

Remark 7 One of the fundamental ingredients in the above proof is the $L^{2}$-continuity in space of the solid velocity contributions and the intrinsic control of (66) provided by the splitting scheme. This $L^{2}$-continuity is guaranteed by the choice of the solid velocity projection operator (44), which distinguishes the cases $\alpha_{1}=0$ and $\alpha_{1}>0$. Note that the case $\alpha_{1}>0$ cannot be handled by considering also the standard Lagrange interpolant $\boldsymbol{I}_{h}^{\Omega^{\mathrm{s}}}$ since, with this choice, the term $T_{3,2}\left(\tau \dot{\boldsymbol{\xi}}^{n-\frac{1}{2}}\right)$ breaks the $L^{2}$-continuity.

Theorem 6 provides a bound of $\mathcal{Z}_{h}^{n}$ whose leading contribution involves $h$-dependent term

$$
\left\|\boldsymbol{L}_{h}^{\mathrm{e}} \boldsymbol{d}+\boldsymbol{L}_{h}^{\mathrm{v}} \boldsymbol{P}_{h}^{\mathrm{s}}(\boldsymbol{u}, \dot{\boldsymbol{d}})\right\|_{H^{r}\left(0, T ; L^{2}\left(\Omega^{\mathrm{s}}\right)\right)} .
$$

The key arguments for the estimation of this quantity, in terms of $h$, are given in the next section.

\subsection{Stability of the discrete viscoelastic operator}

We consider the following notation for the continuous viscoelastic operators

$$
\boldsymbol{L}^{\mathrm{e}} \boldsymbol{d} \stackrel{\text { def }}{=}-\operatorname{div} \boldsymbol{\sigma}(\boldsymbol{d}), \quad \boldsymbol{L}^{\mathrm{v}} \dot{\boldsymbol{d}} \stackrel{\text { def }}{=} \alpha_{0} \rho^{\mathrm{s}} \dot{\boldsymbol{d}}-\alpha_{1} \operatorname{div} \boldsymbol{\sigma}(\dot{\boldsymbol{d}}),
$$

with $\alpha_{0}, \alpha_{1} \geq 0$. The next result describes the behavior of (76) in terms of $h$.

Lemma 7 Let $(\boldsymbol{u}, \dot{\boldsymbol{d}}) \in \boldsymbol{W} \cap\left(\left[H^{2}\left(\Omega^{\mathrm{f}}\right)\right]^{d} \times\left[H^{2}\left(\Omega^{\mathrm{s}}\right)\right]^{d}\right)$. The following estimates hold,

- for $\alpha_{1}=0$ :

$$
\begin{aligned}
\left\|\boldsymbol{L}_{h}^{\mathrm{e}} \boldsymbol{d}+\boldsymbol{L}_{h}^{\mathrm{v}} \boldsymbol{P}_{h}^{\mathrm{s}}(\boldsymbol{u}, \dot{\boldsymbol{d}})\right\|_{0, \Omega^{\mathrm{s}}} \lesssim\left\|\boldsymbol{L}^{\mathrm{e}} \boldsymbol{d}+\boldsymbol{L}^{\mathrm{v}} \dot{\boldsymbol{d}}\right\|_{0, \Omega^{\mathrm{s}}}+h^{-\frac{1}{2}}\left\|\boldsymbol{\sigma}(\boldsymbol{d}) \boldsymbol{n}^{\mathrm{s}}\right\|_{0, \Sigma} \\
+\alpha_{0} \rho^{\mathrm{s}}\left(h\|\dot{\boldsymbol{d}}\|_{1, \Omega^{\mathrm{s}}}+h^{2}\|\dot{\boldsymbol{d}}\|_{2, \Omega^{\mathrm{s}}}\right)
\end{aligned}
$$

- for $\alpha_{1}>0$ :

$$
\begin{aligned}
\left\|\boldsymbol{L}_{h}^{\mathrm{e}} \boldsymbol{d}+\boldsymbol{L}_{h}^{\mathrm{v}} \boldsymbol{P}_{h}^{\mathrm{s}}(\boldsymbol{u}, \dot{\boldsymbol{d}})\right\|_{0, \Omega^{\mathrm{s}}} \lesssim & \left\|\boldsymbol{L}^{\mathrm{e}} \boldsymbol{d}+\boldsymbol{L}^{\mathrm{v}} \dot{\boldsymbol{d}}\right\|_{0, \Omega^{\mathrm{s}}}+h^{-\frac{1}{2}}\left\|\boldsymbol{\sigma}^{\mathrm{s}}(\boldsymbol{d}, \dot{\boldsymbol{d}}) \boldsymbol{n}^{\mathrm{s}}\right\|_{0, \Sigma} \\
& +\left(\frac{\alpha_{0} \rho^{\mathrm{s}} C_{\mathrm{P}}}{\left(\alpha_{1} \alpha_{\mathrm{e}}\right)^{\frac{1}{2}}} h+\left(\alpha_{1} \beta_{\mathrm{e}}\right)^{\frac{1}{2}}\right)\left(\mu^{\frac{1}{2}}\|\boldsymbol{u}\|_{2, \Omega^{\mathrm{f}}}+\left(\alpha_{1} \beta_{\mathrm{e}}\right)^{\frac{1}{2}}\|\dot{\boldsymbol{d}}\|_{2, \Omega^{\mathrm{s}}}\right) \\
& +\frac{\alpha_{0} \rho^{\mathrm{s}} h}{\left(\alpha_{1} \alpha_{\mathrm{e}}\right)^{\frac{1}{2}}}\left(\|\boldsymbol{u}\|_{\boldsymbol{V}^{\mathrm{f}}}+\alpha_{1}^{\frac{1}{2}}\|\dot{\boldsymbol{d}}\|_{\mathrm{e}}\right) .
\end{aligned}
$$

$\mathrm{RR} \mathrm{n}^{\circ} 8670$ 
Proof 7 From (7), (8) and (28), we have

$$
\begin{aligned}
\left(\boldsymbol{L}_{h}^{\mathrm{e}} \boldsymbol{d}+\boldsymbol{L}_{h}^{\mathrm{v}} \boldsymbol{P}_{h}^{\mathrm{s}}(\boldsymbol{u}, \dot{\boldsymbol{d}}), \boldsymbol{v}_{h}^{\mathrm{s}}\right)_{*}= & a^{\mathrm{e}}\left(\boldsymbol{d}, \boldsymbol{v}_{h}^{\mathrm{s}}\right)+\alpha_{0} \rho^{\mathrm{s}}\left(\boldsymbol{P}_{h}^{\mathrm{s}}(\boldsymbol{u}, \dot{\boldsymbol{d}}), \boldsymbol{v}_{h}^{\mathrm{s}}\right)_{\Omega^{\mathrm{s}}}+\alpha_{1} a^{\mathrm{e}}\left(\boldsymbol{P}_{h}^{\mathrm{s}}(\boldsymbol{u}, \dot{\boldsymbol{d}}), \boldsymbol{v}_{h}^{\mathrm{s}}\right) \\
& -\alpha_{0} \rho^{\mathrm{s}} \delta_{h}\left(\boldsymbol{P}_{h}^{\mathrm{s}}(\boldsymbol{u}, \dot{\boldsymbol{d}}), \boldsymbol{v}_{h}^{\mathrm{s}}\right) \\
= & a^{\mathrm{e}}\left(\boldsymbol{d}, \boldsymbol{v}_{h}^{\mathrm{s}}\right)+a^{\mathrm{v}}\left(\dot{\boldsymbol{d}}, \boldsymbol{v}_{h}^{\mathrm{s}}\right)+\alpha_{0} \rho^{\mathrm{s}}\left(\boldsymbol{P}_{h}^{\mathrm{s}}(\boldsymbol{u}, \dot{\boldsymbol{d}})-\dot{\boldsymbol{d}}, \boldsymbol{v}_{h}^{\mathrm{s}}\right)_{\Omega^{\mathrm{s}}}+\alpha_{1} a^{\mathrm{e}}\left(\boldsymbol{P}_{h}^{\mathrm{s}}(\boldsymbol{u}, \dot{\boldsymbol{d}})-\dot{\boldsymbol{d}}, \boldsymbol{v}_{h}^{\mathrm{s}}\right) \\
& -\alpha_{0} \rho^{\mathrm{s}} \delta_{h}\left(\boldsymbol{P}_{h}^{\mathrm{s}}(\boldsymbol{u}, \dot{\boldsymbol{d}}), \boldsymbol{v}_{h}^{\mathrm{s}}\right) .
\end{aligned}
$$

Thus, owing to (29)-(30) and using an inverse inequality (see, e.g., [5, Chapter 4]), it follows that

$$
\begin{aligned}
\left(\boldsymbol{L}_{h}^{\mathrm{e}} \boldsymbol{d}+\boldsymbol{L}_{h}^{\mathrm{v}} \boldsymbol{P}_{h}^{\mathrm{s}}(\boldsymbol{u}, \dot{\boldsymbol{d}}), \boldsymbol{v}_{h}^{\mathrm{s}}\right)_{*} \lesssim & a^{\mathrm{e}}\left(\boldsymbol{d}, \boldsymbol{v}_{h}^{\mathrm{s}}\right)+a^{\mathrm{v}}\left(\dot{\boldsymbol{d}}, \boldsymbol{v}_{h}^{\mathrm{s}}\right)+\alpha_{0} \rho^{\mathrm{s}}\left\|\boldsymbol{P}_{h}^{\mathrm{s}}(\boldsymbol{u}, \dot{\boldsymbol{d}})-\dot{\boldsymbol{d}}\right\|_{0, \Omega^{\mathrm{s}}}\left\|\boldsymbol{v}_{h}^{\mathrm{s}}\right\|_{0, \Omega^{\mathrm{s}}} \\
& +\alpha_{1} \frac{\beta_{\mathrm{e}}^{\frac{1}{2}}}{h}\left\|\boldsymbol{P}_{h}^{\mathrm{s}}(\boldsymbol{u}, \dot{\boldsymbol{d}})-\dot{\boldsymbol{d}}\right\|_{\mathrm{e}}\left\|\boldsymbol{v}_{h}^{\mathrm{s}}\right\|_{0, \Omega^{\mathrm{s}}}+\alpha_{0} \rho^{\mathrm{s}} h\left\|\boldsymbol{P}_{h}^{\mathrm{s}}(\boldsymbol{u}, \dot{\boldsymbol{d}})\right\|_{1, \Omega^{\mathrm{s}}}\left\|\boldsymbol{v}_{h}^{\mathrm{s}}\right\|_{0, \Omega^{\mathrm{s}}} .
\end{aligned}
$$

In addition, by using the definition (4), integration by parts, the boundary conditions on $\Gamma^{\mathrm{s}, \mathrm{d}} \cup \Gamma^{\mathrm{s}, \mathrm{n}}$ and a discrete trace inequality (see, e.g., [5, Chapter 10]), we get

$$
\begin{aligned}
a^{\mathrm{e}}\left(\boldsymbol{d}, \boldsymbol{v}_{h}^{\mathrm{s}}\right)+a^{\mathrm{v}}\left(\dot{\boldsymbol{d}}, \boldsymbol{v}_{h}^{\mathrm{s}}\right) & =\left(\boldsymbol{\sigma}(\boldsymbol{d}), \boldsymbol{\epsilon}\left(\boldsymbol{v}_{h}^{\mathrm{s}}\right)\right)_{\Omega^{\mathrm{s}}}+\alpha_{0} \rho^{\mathrm{s}}\left(\dot{\boldsymbol{d}}, \boldsymbol{v}_{h}^{\mathrm{s}}\right)_{\Omega^{\mathrm{s}}}+\alpha_{1}\left(\boldsymbol{\sigma}(\dot{\boldsymbol{d}}), \boldsymbol{\epsilon}\left(\boldsymbol{v}_{h}^{\mathrm{s}}\right)\right)_{\Omega^{\mathrm{s}}} \\
& =\left(\boldsymbol{L}^{\mathrm{e}} \boldsymbol{d}+\boldsymbol{L}^{\mathrm{v}} \dot{\boldsymbol{d}}, \boldsymbol{v}_{h}^{\mathrm{s}}\right)_{\Omega^{\mathrm{s}}}+\int_{\Sigma} \boldsymbol{\sigma}^{\mathrm{s}}(\boldsymbol{d}, \dot{\boldsymbol{d}}) \boldsymbol{n}^{\mathrm{s}} \cdot \boldsymbol{v}_{h}^{\mathrm{s}} \\
& \lesssim\left(\left\|\boldsymbol{L}^{\mathrm{e}} \boldsymbol{d}+\boldsymbol{L}^{\mathrm{v}} \dot{\boldsymbol{d}}\right\|_{0, \Omega^{\mathrm{s}}}+h^{-\frac{1}{2}}\left\|\boldsymbol{\sigma}^{\mathrm{s}}(\boldsymbol{d}, \dot{\boldsymbol{d}}) \boldsymbol{n}^{\mathrm{s}}\right\|_{0, \Sigma}\right)\left\|\boldsymbol{v}_{h}^{\mathrm{s}}\right\|_{0, \Omega^{\mathrm{s}}}
\end{aligned}
$$

As a result, by inserting this estimate into (79), we get

$$
\begin{aligned}
& \left(\boldsymbol{L}_{h}^{\mathrm{e}} \boldsymbol{d}+\boldsymbol{L}_{h}^{\mathrm{v}} \boldsymbol{P}_{h}^{\mathrm{s}}(\boldsymbol{u}, \dot{\boldsymbol{d}}), \boldsymbol{v}_{h}^{\mathrm{s}}\right)_{*} \lesssim\left(\left\|\boldsymbol{L}^{\mathrm{e}} \boldsymbol{d}+\boldsymbol{L}^{\mathrm{v}} \dot{\boldsymbol{d}}\right\|_{0, \Omega^{\mathrm{s}}}+h^{-\frac{1}{2}}\left\|\boldsymbol{\sigma}^{\mathrm{s}}(\boldsymbol{d}, \dot{\boldsymbol{d}}) \boldsymbol{n}^{\mathrm{s}}\right\|_{0, \Sigma}\right. \\
& \left.\quad+\alpha_{0} \rho^{\mathrm{s}}\left\|\boldsymbol{P}_{h}^{\mathrm{s}}(\boldsymbol{u}, \dot{\boldsymbol{d}})-\dot{\boldsymbol{d}}\right\|_{0, \Omega^{\mathrm{s}}}+\alpha_{1} \frac{\beta_{\mathrm{e}}^{\frac{1}{2}}}{h}\left\|\boldsymbol{P}_{h}^{\mathrm{s}}(\boldsymbol{u}, \dot{\boldsymbol{d}})-\dot{\boldsymbol{d}}\right\|_{\mathrm{e}}+\alpha_{0} \rho^{\mathrm{s}} h\left\|\boldsymbol{P}_{h}^{\mathrm{s}}(\boldsymbol{u}, \dot{\boldsymbol{d}})\right\|_{1, \Omega^{\mathrm{s}}}\right)\left\|\boldsymbol{v}_{h}^{\mathrm{s}}\right\|_{0, \Omega^{\mathrm{s}}}
\end{aligned}
$$

The estimate (77) then follows by taking $\boldsymbol{v}_{h}^{\mathrm{s}}=\boldsymbol{L}_{h}^{\mathrm{e}} \boldsymbol{d}+\boldsymbol{L}_{h}^{\mathrm{v}} \boldsymbol{P}_{h}^{\mathrm{s}}(\boldsymbol{u}, \dot{\boldsymbol{d}})$ in (81) and thereafter using (44) and (38). At last, the estimate (78) follows by a similar argument together with Poincaré inequality and Lemma 3.

Lemma 7 predicts an $\mathcal{O}\left(h^{-\frac{1}{2}}\right)$ behavior for the quantities (76). This is due to the thick-walled nature of the solid which induces a non-uniformity in $h$ of the discrete operators $\boldsymbol{L}_{h}^{\mathrm{e}}$ and $\boldsymbol{L}_{h}^{\mathrm{v}}$. Basically, the $h^{\frac{1}{2}}$-loss comes from the interface terms arising in the integration by parts of $(80)$.

Remark 8 It should be noted that the above issue does not appear in the case of coupling with a thin-walled structure (as shown in [1\%, 20]), since by construction the fluid-structure interface $\Sigma$ and the solid domain $\Omega^{\mathrm{s}}$ coincide. This explains the differences on the convergence rates observed in [20, Section 6.1] and [21, Section 6.1.1]. 


\subsection{A priori error estimates}

For $n>r$, we define the energy norm of the error at time $t_{n}$ as

$$
\begin{aligned}
\mathcal{E}_{h}^{n} \stackrel{\text { def }}{=}\left(\rho^{\mathrm{f}}\right)^{\frac{1}{2}}\left\|\boldsymbol{u}^{n}-\boldsymbol{u}_{h}^{n}\right\|_{0, \Omega^{\mathrm{f}}} & +\left(\sum_{m=r+1}^{n} \tau\left\|\boldsymbol{u}^{m}-\boldsymbol{u}_{h}^{m}\right\|_{\boldsymbol{V}}^{2}\right)^{\frac{1}{2}}+\left(\sum_{m=r+1}^{n} \tau\left|p_{h}^{m}\right|_{s_{h}}^{2}\right)^{\frac{1}{2}} \\
& +\left(\rho^{\mathrm{s}}\right)^{\frac{1}{2}}\left\|\dot{\boldsymbol{d}}^{n}-\dot{\boldsymbol{d}}_{h}^{n}\right\|_{0, \Omega^{\mathrm{s}}}+\left\|\boldsymbol{d}^{n}-\boldsymbol{d}_{h}^{n}\right\|_{\mathrm{e}}+\left(\sum_{m=r+1}^{n} \tau\left\|\dot{\boldsymbol{d}}^{m}-\dot{\boldsymbol{d}}_{h}^{m}\right\|_{\mathrm{v}}^{2}\right)^{\frac{1}{2}} .
\end{aligned}
$$

The next result provides a priori error estimates for Algorithms 1 and 2 .

Theorem 8 Under the assumptions of Theorem 6 and if, for $\alpha_{1}>0$,

$$
\boldsymbol{u} \in\left[H^{r}\left(0, T ; H^{2}\left(\Omega^{\mathrm{f}}\right)\right)\right]^{d}, \quad \dot{\boldsymbol{d}} \in\left[H^{r}\left(0, T ; H^{2}\left(\Omega^{\mathrm{s}}\right)\right)\right]^{d},
$$

the following error estimates hold,

- for $\alpha_{1}=0$ :

$$
\begin{aligned}
\mathcal{E}_{h}^{n} \lesssim \tilde{c}_{1} h+ & \tilde{c}_{2} \tau+\tilde{c}_{3} \tau^{2^{r-1}}\left(\tilde{c}_{4} h+\tilde{c}_{5} h^{2}\right) \\
& +\tilde{c}_{3} \tau^{2^{r-1}}\left(\left\|\boldsymbol{L}^{\mathrm{e}} \boldsymbol{d}+\boldsymbol{L}^{\mathrm{v}} \dot{\boldsymbol{d}}\right\|_{H^{r}\left(0, T ; L^{2}\left(\Omega^{\mathrm{s}}\right)\right)}+h^{-\frac{1}{2}}\left\|\boldsymbol{\sigma}(\boldsymbol{d}) \boldsymbol{n}^{\mathrm{s}}\right\|_{H^{r}\left(0, T ; L^{2}(\Sigma)\right)}\right)
\end{aligned}
$$

- for $\alpha_{1}>0$ :

$$
\begin{aligned}
\mathcal{E}_{h}^{n} \lesssim & \tilde{c}_{6}\left(1+\tilde{c}_{7} \alpha_{1}^{-\frac{1}{2}}\right) h+\tilde{c}_{8} \tau+\tilde{c}_{9} \tau^{2^{r-1}}\left(\tilde{c}_{10} h+\tilde{c}_{11} h \alpha_{1}^{-\frac{1}{2}}+\tilde{c}_{12} \alpha_{1}^{\frac{1}{2}}+\tilde{c}_{13} \alpha_{1}\right) \\
& +\tilde{c}_{9} \tau^{2^{r-1}}\left(\left\|\boldsymbol{L}^{\mathrm{e}} \boldsymbol{d}+\boldsymbol{L}^{\mathrm{v}} \dot{\boldsymbol{d}}\right\|_{H^{r}\left(0, T ; L^{2}\left(\Omega^{\mathrm{s}}\right)\right)}+h^{-\frac{1}{2}}\left\|\boldsymbol{\sigma}^{\mathrm{s}}(\boldsymbol{d}, \dot{\boldsymbol{d}}) \boldsymbol{n}^{\mathrm{s}}\right\|_{H^{r}\left(0, T ; L^{2}(\Sigma)\right)}\right)
\end{aligned}
$$

Here, the symbols $\left\{\tilde{c}_{i}\right\}_{i=1}^{13}$ denote positive constants independent of $h, \tau$ and $\alpha_{1}$, but which depend on other physical parameters and on the regularity of $(\boldsymbol{u}, p, \boldsymbol{d}, \dot{\boldsymbol{d}})$.

Proof 8 The above estimates straightforwardly follow from the results of Theorem 6 and Lemma $\%$, in combination with the approximation results and the error decomposition of Sections 4.2-4.3.

Theorem 8 confirms the numerical evidence reported in [21] for Algorithm 2. Another salient consequence of Theorem 8 is that the mass-lumping approximation does not perturbs the overall accuracy of the coupling scheme, since the truncation error induced by splitting is the same for Algorithms 1 and 2 .

Provided that the exact solution has the appropriate regularity, the error introduced by Algorithms 1 and 2 has an order $\mathcal{O}\left((\tau / h)^{\frac{1}{2}}\right)$ if $r=0$. In this case, the standard hyperbolicCFL scaling $\tau=\mathcal{O}(h)$ is not enough to guarantee the convergence of the scheme. Furthermore, a sub-optimal convergence rate is recovered for the scheme with $r=0$ under the restrictive parabolic-CFL condition $\tau=\mathcal{O}\left(h^{2}\right)$.

Remark 9 In the particular case of the coupling with a purely elastic structure (i.e., $\alpha_{0}=\alpha_{1}=$ $0)$, similar conclusions hold for Algorithm 3 with $\beta=0$. Indeed, in this framework, the method coincides with the non-extrapolated variant of Algorithm 1 and, hence, the error estimate (82) applies with $r=0$.

$\mathrm{RR} \mathrm{n}^{\circ} 8670$ 
With the first-order extrapolation $(r=1)$, the leading term of the error is of order $\mathcal{O}\left(\tau / h^{\frac{1}{2}}\right)$. This means that a sub-optimal convergence rate is obtained under $\tau=\mathcal{O}(h)$. An overall firstorder convergence rate is guaranteed under a $\frac{3}{2}-\mathrm{CFL}$ condition, $\tau=\mathcal{O}\left(h^{\frac{3}{2}}\right)$. We recall that, owing to Theorem 2, Algorithms 1 and 2 are unconditionally stable in this case.

At last, with $r=2$, the error introduced by the splitting is of order $\mathcal{O}\left(\tau^{2} / h^{\frac{1}{2}}\right)$, which is enough to ensure an optimal first-order convergence rate under $\tau=\mathcal{O}(h)$, but the stability condition (24) demands further restrictions on the discretization parameters.

\section{$5 \quad$ Numerical experiments}

As discussed above, numerical evidence on the convergence rates of Algorithm 2 has already been reported in [21, Section 6.1.1]. The objective of this numerical section is twofold:

- The first target is to illustrate that, as predicted by the error estimates of Theorem 8 , Algorithms 1 and 2 deliver the same convergence rate. In order words, the mass-lumping approximation does not affect the accuracy of the splitting;

- The second is to demonstrate that Algorithm 3 also suffers from similar non-uniform convergence issues.

To this purpose we consider the pressure wave numerical example of [21, Section 6.1.1], namely, in (1)-(3) we take $\Omega^{\mathrm{f}}=[0, L] \times[0, R], \Omega^{\mathrm{s}}=[0, L] \times[R, R+\epsilon], L=6, R=0.5$ and $\epsilon=0.1$. All the units are given in the CGS system. At the fluid boundary $x=0$ we impose a sinusoidal pressure of maximal amplitude $2 \times 10^{4}$ during $5 \times 10^{-3}$ time instants, corresponding to half a period. Zero traction is enforced at $x=6$ and a symmetry condition is applied on the lower wall. Transverse membrane effects that appear in axisymmetric formulations are included through an additional zeroth-order term $c_{0} \boldsymbol{d}$ in the solid equation (2) $)_{1}$. The solid is clamped at its extremities and zero traction is enforced on its upper boundary. The fluid physical parameters are given by $\rho^{\mathrm{f}}=1$ and $\mu=0.035$. For the solid we have $\rho^{\mathrm{s}}=1.1, L_{1}=1.15 \cdot 10^{6}, L_{2}=1.7 \cdot 10^{6}$ and $c_{0}=4 \cdot 10^{6}$. For the sake of simplicity, a purely elastic solid is considered $\left(\alpha_{0}=\alpha_{1}=0\right)$. The pressure stabilization parameter in (35) is set to $\kappa=10^{-3}$. All the computations have been performed with FreeFem ++ (see [27]).

We have reported the relative displacement error $\left\|\boldsymbol{d}_{h}^{N}-\boldsymbol{d}(T)\right\|_{\mathrm{e}} /\|\boldsymbol{d}(T)\|_{\mathrm{e}}$ at the final time $T=0.015$ for the different schemes and several choices of scaling for the couple $(h, \tau)$. We also present the rate of convergence to illustrate the global accuracy of the schemes. Relative errors are calculated with a reference solution obtained with an implicit coupling scheme with $h=0.003125$ and $\tau=10^{-6}$.

Table 1 reports the convergence results for Algorithm 2 with $\tau=\mathcal{O}(h)$. We clearly see that the scheme with $r=0$ does not converge and that the scheme with $r=1$ exhibits a sub-optimal convergence rate. As presented in Table 2, same phenomena are observed with Algorithms 1. This points out the fact the mass-lumping approximation has nothing to do with the non-uniformity in $h$ of the splitting. Table 3 demonstrates that Algorithm 3 with $\beta=1$ also suffers from this issue. We recall that, since the structure is purely elastic, Algorithm 3 with $\beta=0$ coincides with Algorithm 1 with $r=0$.

We now consider a different scaling for the couple of discretization parameters. Table 4 reports the results obtained with Algorithms 1 and 2 with $r=1$ and $\tau=\mathcal{O}\left(h^{\frac{3}{2}}\right)$. In both cases we recover an optimal first-order convergence rate. In Table 5 we report the convergence results for Algorithms 1 and 2 with $r=2$ and $\tau=\mathcal{O}\left(h^{\frac{6}{5}}\right)$. Note that, in this case, the scaling $\tau=\mathcal{O}(h)$ is inappropriate since the stability condition (24) is not fulfilled. We clearly see that both schemes deliver the same accuracy. Note that with the finest discretization we obtain a convergence rate 


\begin{tabular}{cc|cc|cc}
\hline \hline & & \multicolumn{2}{|c|}{$r=0$} & \multicolumn{2}{c}{$r=1$} \\
$h$ & $\tau$ & rel. error & $h$-rate & rel. error & $h$-rate \\
\hline 0.1 & $3.75 \times 10^{-4}$ & 1.00 & - & 0.96 & - \\
0.05 & $1.875 \times 10^{-4}$ & 1.00 & 0 & 0.83 & 0.22 \\
0.025 & $9.375 \times 10^{-5}$ & 1.00 & 0 & 0.57 & 0.54 \\
0.0125 & $4.6875 \times 10^{-5}$ & 1.01 & 0 & 0.33 & 0.77 \\
0.00625 & $2.34375 \times 10^{-5}$ & 1.01 & 0 & 0.18 & 0.88 \\
\hline
\end{tabular}

Table 1: Algorithm 2 with $\tau=\mathcal{O}(h)$.

\begin{tabular}{|c|c|c|c|c|c|}
\hline \multirow[b]{2}{*}{$h$} & \multirow[b]{2}{*}{$\tau$} & \multicolumn{2}{|c|}{$r=0$} & \multicolumn{2}{|c|}{$r=1$} \\
\hline & & rel. error & $h$-rate & rel. error & $h$-rate \\
\hline 0.1 & $3.75 \times 10^{-4}$ & 1.00 & - & 1.11 & - \\
\hline 0.05 & $1.875 \times 10^{-4}$ & 1.00 & 0 & 1.07 & 0.05 \\
\hline 0.025 & $9.375 \times 10^{-5}$ & 1.00 & 0 & 0.83 & 0.37 \\
\hline 0.0125 & $4.6875 \times 10^{-5}$ & 1.00 & 0 & 0.52 & 0.67 \\
\hline 0.00625 & $2.34375 \times 10^{-5}$ & 1.00 & 0 & 0.29 & 0.83 \\
\hline
\end{tabular}

Table 2: Algorithm 1 with $\tau=\mathcal{O}(h)$.

\begin{tabular}{cccc}
\hline \hline$h$ & $\tau$ & rel. error & $h$-rate \\
\hline 0.1 & $3.75 \times 10^{-4}$ & 1.06899 & - \\
0.05 & $1.875 \times 10^{-4}$ & 1.01822 & 0.07 \\
0.025 & $9.375 \times 10^{-5}$ & 0.79880 & 0.35 \\
0.0125 & $4.6875 \times 10^{-5}$ & 0.51719 & 0.64 \\
0.00625 & $2.34375 \times 10^{-5}$ & 0.29598 & 0.82 \\
\hline
\end{tabular}

Table 3: Algorithm 3 with $\beta=1$ and $\tau=\mathcal{O}(h)$.

\begin{tabular}{cc|cc|cc}
\hline \hline & & \multicolumn{2}{|c|}{ Algorithm 1 } & \multicolumn{2}{c}{ Algorithm 2 } \\
$h$ & $\tau$ & rel. error & $h$-rate & rel. error & $h$-rate \\
\hline 0.1 & $3.75 \times 10^{-4}$ & 0.96 & - & 1.11 & - \\
0.05 & $1.60 \times 10^{-4}$ & 0.70 & 0.47 & 0.92 & 0.03 \\
0.025 & $6.87 \times 10^{-5}$ & 0.36 & 0.95 & 0.52 & 0.83 \\
0.0125 & $2.93749 \times 10^{-5}$ & 0.16 & 1.21 & 0.23 & 1.18 \\
\hline
\end{tabular}

Table 4: Algorithm 1 and 2 with $r=1$ and $\tau=\mathcal{O}\left(h^{\frac{3}{2}}\right)$.

larger than one. This is certainly due to the fact that we are too close to the resolution with which the reference solution has been generated.

\section{Conclusion}

We have performed an a priori error analysis of the generalized Robin-Neumann explicit coupling schemes introduced in $[19,21]$. The schemes have been derived within a fractional-step splitting 


\begin{tabular}{cc|cc|cc}
\hline \hline & & \multicolumn{2}{|c|}{ Algorithm 1 } & \multicolumn{2}{c}{ Algorithm 2 } \\
$h$ & $\tau$ & rel. error & $h$-rate & rel. error & $h$-rate \\
\hline 0.1 & $3 \times 10^{-5}$ & 0.73496 & - & 0.73546 & - \\
0.05 & $1.40 \times 10^{-5}$ & 0.38805 & 0.92 & 0.38978 & 0.92 \\
0.025 & $6.57 \times 10^{-6}$ & 0.14419 & 1.42 & 0.14578 & 1.42 \\
0.0125 & $3.07 \times 10^{-6}$ & 0.04197 & 1.78 & 0.04255 & 1.78 \\
\hline
\end{tabular}

Table 5: Algorithm 1 and 2 with $r=2$ and $\tau=\mathcal{O}\left(h^{6 / 5}\right)$.

framework, which covers both the case with and without mass-lumping approximation in the solid. In both cases, the analysis confirms the $\mathcal{O}\left(\tau^{2^{r-1}} / h^{\frac{1}{2}}\right)$ error perturbation anticipated by the numerical evidence of [21] for the variant with mass-lumping in the solid. Another fundamental result of this work is that the $h$-non-uniformity of the splitting error is not a consequence of the mass-lumping approximation, which simply dictates the explicit or semi-implicit nature of the coupling scheme. The analysis indicates that the genesis of the $h^{\frac{1}{2}}$-loss is the non-uniformity of the discrete elastic or viscoelastic operator, induced by the thick-walled nature of the solid.

\section{Acknowledgements}

This work was supported by the French National Research Agency (ANR) through the EXIFSI project (ANR-12-JS01-0004).

\section{A Proof of Lemma 3}

We proceed by extending the arguments reported in [25] and [17]. We first prove the following generalized inf-sup condition.

Lemma 9 There holds

$$
\left\|q_{h}\right\|_{Q} \lesssim \sup _{\left(\boldsymbol{v}_{h}^{\mathrm{f}}, \boldsymbol{v}_{h}^{\mathrm{s}}\right) \in \boldsymbol{W}_{h} \backslash\{\mathbf{0}\}} \frac{b\left(q_{h}, \boldsymbol{v}_{h}^{\mathrm{f}}\right)}{\left\|\boldsymbol{v}_{h}^{\mathrm{f}}\right\|_{\boldsymbol{V}}+\alpha_{1}^{\frac{1}{2}}\left\|\boldsymbol{v}_{h}^{\mathrm{s}}\right\|_{\mathrm{e}}}+\left|q_{h}\right|_{s_{h}}
$$

for all $q_{h} \in Q_{h}$.

Proof 9 From [1\%, Lemma 4] we know that

$$
\left\|q_{h}\right\|_{Q} \lesssim \sup _{\boldsymbol{v}_{h}^{\mathrm{f}} \in \boldsymbol{V}_{h}^{\mathrm{f}} \backslash\{\mathbf{0}\}} \frac{b\left(q_{h}, \boldsymbol{v}_{h}^{\mathrm{f}}\right)}{\left\|\boldsymbol{v}_{h}^{\mathrm{f}}\right\|_{\boldsymbol{V}}}+\left|q_{h}\right|_{s_{h}}
$$

for all $q_{h} \in Q_{h}$. Therefore, it only remains to prove that

$$
\sup _{\boldsymbol{v}_{h}^{\mathrm{f}} \in \boldsymbol{V}_{h}^{\mathrm{f}} \backslash\{\mathbf{0}\}} \frac{b\left(q_{h}, \boldsymbol{v}_{h}^{\mathrm{f}}\right)}{\left\|\boldsymbol{v}_{h}^{\mathrm{f}}\right\|_{\boldsymbol{V}}} \lesssim \sup _{\left(\boldsymbol{v}_{h}^{\mathrm{f}}, \boldsymbol{v}_{h}^{\mathrm{s}}\right) \in \boldsymbol{W}_{h} \backslash\{\mathbf{0}\}} \frac{b\left(q_{h}, \boldsymbol{v}_{h}^{\mathrm{f}}\right)}{\left\|\boldsymbol{v}_{h}^{\mathrm{f}}\right\|_{\boldsymbol{V}}+\alpha_{1}^{\frac{1}{2}}\left\|\boldsymbol{v}_{h}^{\mathrm{s}}\right\|_{\mathrm{e}}}
$$

for all $q_{h} \in Q_{h}$. More precisely, let $q_{h} \in Q_{h}$ and $\boldsymbol{v}_{h}^{\mathrm{f}} \in \boldsymbol{V}_{h}^{\mathrm{f}} \backslash\{\mathbf{0}\}$, it is sufficient to find $\boldsymbol{v}_{h}^{\mathrm{s}} \in \boldsymbol{V}_{h}^{\mathrm{s}}$ satisfying $\left(\boldsymbol{v}_{h}^{\mathrm{f}}, \boldsymbol{v}_{h}^{\mathrm{s}}\right) \in \boldsymbol{W}_{h}^{\mathrm{s}}$ and

$$
\frac{b\left(q_{h}, \boldsymbol{v}_{h}^{\mathrm{f}}\right)}{\left\|\boldsymbol{v}_{h}^{\mathrm{f}}\right\|_{\boldsymbol{V}}} \lesssim \frac{b\left(q_{h}, \boldsymbol{v}_{h}^{\mathrm{f}}\right)}{\left\|\boldsymbol{v}_{h}^{\mathrm{f}}\right\|_{\boldsymbol{V}}+\alpha_{1}^{\frac{1}{2}}\left\|\boldsymbol{v}_{h}^{\mathrm{s}}\right\|_{\mathrm{e}}}
$$


To this purpose, we set $\boldsymbol{v}_{h}^{\mathrm{s}}=\boldsymbol{E}_{h}\left(\left.\boldsymbol{v}_{h}^{\mathrm{f}}\right|_{\Sigma}\right)$, where $\boldsymbol{E}_{h}: \Lambda_{\Sigma, h} \rightarrow \boldsymbol{V}_{h}^{\mathrm{s}}$ denotes a uniformly bounded discrete lifting operator (see, e.g., [14, Theorem 2.2]), viz.,

$$
\left.\left(\boldsymbol{E}_{h} \boldsymbol{\xi}_{h}\right)\right|_{\Sigma}=\boldsymbol{\xi}_{h}, \quad\left\|\boldsymbol{E}_{h} \boldsymbol{\xi}_{h}\right\|_{1, \Omega^{\mathrm{s}}} \lesssim\left\|\boldsymbol{\xi}_{h}\right\|_{\frac{1}{2}, \Sigma}
$$

for all $\boldsymbol{\xi}_{h} \in \Lambda_{\Sigma, h}$. Hence, owing to (30) and to the continuity of the trace operator, we have

$$
\alpha_{1}\left\|\boldsymbol{v}_{h}^{\mathrm{s}}\right\|_{\mathrm{e}}^{2} \leq \alpha_{1} \beta_{\mathrm{e}}\left\|\boldsymbol{v}_{h}^{\mathrm{s}}\right\|_{1, \Omega^{\mathrm{s}}}^{2} \lesssim \alpha_{1} \beta_{\mathrm{e}}\left\|\boldsymbol{v}_{h}^{\mathrm{f}}\right\|_{\frac{1}{2}, \Sigma}^{2} \lesssim \alpha_{1} \beta_{\mathrm{e}}\left\|\boldsymbol{v}_{h}^{\mathrm{f}}\right\|_{1, \Omega^{\mathrm{f}}}^{2} \lesssim \frac{\alpha_{1} \beta_{\mathrm{e}}}{\mu}\left\|\boldsymbol{v}_{h}^{\mathrm{f}}\right\|_{V}^{2},
$$

which completes the proof.

The previous result guarantees the well-posedness of problem (40) (see, e.g., [12, Theorem 3.5]). The energy estimate (42) hence follows by taking

$$
\boldsymbol{v}_{h}^{\mathrm{f}}=\boldsymbol{P}_{h}^{\mathrm{f}}(\boldsymbol{u}, \dot{\boldsymbol{d}}), \quad \boldsymbol{v}_{h}^{\mathrm{s}}=\boldsymbol{P}_{h}^{\mathrm{s}}(\boldsymbol{u}, \dot{\boldsymbol{d}}), \quad q_{h}=R_{h}(\boldsymbol{u}, \dot{\boldsymbol{d}}),
$$

in (40) and applying standard arguments to control the right-hand side.

We now focus on the a priori error estimate (43). To this purpose we first state the following approximation result.

Lemma 10 Let $\left(\left(\boldsymbol{u}_{h}, \dot{\boldsymbol{d}}_{h}\right), p_{h}\right) \in \boldsymbol{W}_{h} \times Q_{h}$ be given by (40). There holds

$$
\begin{aligned}
\left\|\boldsymbol{u}-\boldsymbol{u}_{h}\right\|_{\boldsymbol{V}}+\alpha_{1}^{\frac{1}{2}}\left\|\dot{\boldsymbol{d}}-\dot{\boldsymbol{d}}_{h}\right\|_{\mathrm{e}}+\left\|p_{h}\right\|_{Q}+\left|p_{h}\right|_{s_{h}} & \\
& \lesssim \inf _{\left(\left(\hat{\boldsymbol{v}}_{h}^{\mathrm{f}}, \hat{\boldsymbol{v}}_{h}^{\mathrm{s}}\right), \hat{p}_{h}\right) \in \boldsymbol{Z}_{h}}\left\{\left\|\boldsymbol{u}-\hat{\boldsymbol{v}}_{h}^{\mathrm{f}}\right\|_{\boldsymbol{V}}+\alpha_{1}^{\frac{1}{2}}\left\|\dot{\boldsymbol{d}}-\hat{\boldsymbol{v}}_{h}^{\mathrm{s}}\right\|_{\mathrm{e}}+\left\|\hat{p}_{h}\right\|_{Q}+\left|\hat{p}_{h}\right|_{s_{h}}\right\},
\end{aligned}
$$

where

$$
\boldsymbol{Z}_{h} \stackrel{\text { def }}{=}\left\{\left(\left(\hat{\boldsymbol{v}}_{h}^{\mathrm{f}}, \hat{\boldsymbol{v}}_{h}^{\mathrm{s}}\right), \hat{p}_{h}\right) \in \boldsymbol{W}_{h} \times Q_{h} / b\left(q_{h}, \hat{\boldsymbol{v}}_{h}^{\mathrm{f}}\right)=s_{h}\left(\hat{p}_{h}, q_{h}\right) \quad \forall q_{h} \in Q_{h}\right\} .
$$

Proof 10 Let $\left(\left(\hat{\boldsymbol{v}}_{h}^{\mathrm{f}}, \hat{\boldsymbol{v}}_{h}^{\mathrm{s}}\right), \hat{p}_{h}\right)$ be given in $\boldsymbol{Z}_{h}$, for all $\left(\boldsymbol{w}_{h}^{\mathrm{f}}, \boldsymbol{w}_{h}^{\mathrm{s}}\right) \in \boldsymbol{W}_{h}$, we have

$$
\begin{aligned}
a\left(\boldsymbol{u}_{h}-\hat{\boldsymbol{v}}_{h}^{\mathrm{f}}, \boldsymbol{w}_{h}^{\mathrm{f}}\right)+\alpha_{1} a^{\mathrm{e}}\left(\dot{\boldsymbol{d}}_{h}-\hat{\boldsymbol{v}}_{h}^{\mathrm{s}}, \boldsymbol{w}_{h}^{\mathrm{s}}\right)= & a\left(\boldsymbol{u}_{h}-\boldsymbol{u}, \boldsymbol{w}_{h}^{\mathrm{f}}\right)+\alpha_{1} a^{\mathrm{e}}\left(\dot{\boldsymbol{d}}_{h}-\dot{\boldsymbol{d}}, \boldsymbol{w}_{h}^{\mathrm{s}}\right) \\
& +a\left(\boldsymbol{u}-\hat{\boldsymbol{v}}_{h}^{\mathrm{f}}, \boldsymbol{w}_{h}^{\mathrm{f}}\right)+\alpha_{1} a^{\mathrm{e}}\left(\dot{\boldsymbol{d}}-\hat{\boldsymbol{v}}_{h}^{\mathrm{s}}, \boldsymbol{w}_{h}^{\mathrm{s}}\right) \\
= & -b\left(p_{h}, \boldsymbol{w}_{h}^{\mathrm{f}}\right)+a\left(\boldsymbol{u}-\hat{\boldsymbol{v}}_{h}^{\mathrm{f}}, \boldsymbol{w}_{h}^{\mathrm{f}}\right)+\alpha_{1} a^{\mathrm{e}}\left(\dot{\boldsymbol{d}}-\hat{\boldsymbol{v}}_{h}^{\mathrm{s}}, \boldsymbol{w}_{h}^{\mathrm{s}}\right) \\
= & -b\left(\hat{p}_{h}, \boldsymbol{w}_{h}^{\mathrm{f}}\right)+b\left(\hat{p}_{h}-p_{h}, \boldsymbol{w}_{h}^{\mathrm{f}}\right) \\
& +a\left(\boldsymbol{u}-\hat{\boldsymbol{v}}_{h}^{\mathrm{f}}, \boldsymbol{w}_{h}^{\mathrm{f}}\right)+\alpha_{1} a^{\mathrm{e}}\left(\dot{\boldsymbol{d}}-\hat{\boldsymbol{v}}_{h}^{\mathrm{s}}, \boldsymbol{w}_{h}^{\mathrm{s}}\right) .
\end{aligned}
$$

Then, we take $\boldsymbol{w}_{h}^{\mathrm{f}}=\boldsymbol{u}_{h}-\hat{\boldsymbol{v}}_{h}^{\mathrm{f}}$ and $\boldsymbol{w}_{h}^{\mathrm{s}}=\dot{\boldsymbol{d}}_{h}-\hat{\boldsymbol{v}}_{h}^{\mathrm{s}}$ to get

$$
\begin{aligned}
2 \mu\left\|\boldsymbol{\epsilon}\left(\boldsymbol{u}_{h}-\hat{\boldsymbol{v}}_{h}^{\mathrm{f}}\right)\right\|_{0, \Omega^{\mathrm{f}}}^{2}+\alpha_{1}\left\|\dot{\boldsymbol{d}}_{h}-\hat{\boldsymbol{v}}_{h}^{\mathrm{s}}\right\|_{\mathrm{e}}^{2}= & -b\left(\hat{p}_{h}, \boldsymbol{u}_{h}-\hat{\boldsymbol{v}}_{h}^{\mathrm{f}}\right)-\left|p_{h}-\hat{p}_{h}\right|_{s_{h}}^{2}+a\left(\boldsymbol{u}-\hat{\boldsymbol{v}}_{h}^{\mathrm{f}}, \boldsymbol{u}_{h}-\hat{\boldsymbol{v}}_{h}^{\mathrm{f}}\right) \\
& +\alpha_{1} a^{\mathrm{e}}\left(\dot{\boldsymbol{d}}-\hat{\boldsymbol{v}}_{h}^{\mathrm{s}}, \dot{\boldsymbol{d}}_{h}-\hat{\boldsymbol{v}}_{h}^{\mathrm{s}}\right) .
\end{aligned}
$$

Therefore, by using the Korn inequality (see, e.g., [5, Chapter 11]), the continuity of the bilinear forms $b, a$ and $a^{\mathrm{e}}$ and the Cauchy-Schwarz inequality, it follows that

$$
\left\|\boldsymbol{u}_{h}-\hat{\boldsymbol{v}}_{h}^{\mathrm{f}}\right\|_{\boldsymbol{V}}^{2}+\alpha_{1}\left\|\dot{\boldsymbol{d}}_{h}-\hat{\boldsymbol{v}}_{h}^{\mathrm{s}}\right\|_{\mathrm{e}}^{2}+\left|p_{h}-\hat{p}_{h}\right|_{s_{h}}^{2} \lesssim\left\|\boldsymbol{u}-\hat{\boldsymbol{v}}_{h}^{\mathrm{f}}\right\|_{\boldsymbol{V}}^{2}+\alpha_{1}\left\|\dot{\boldsymbol{d}}-\hat{\boldsymbol{v}}_{h}^{\mathrm{s}}\right\|_{\mathrm{e}}^{2}+\left\|\hat{p}_{h}\right\|_{Q}^{2},
$$

$\mathrm{RR} \mathrm{n}^{\circ} 8670$ 
which, in combination with a triangle inequality, yields

$$
\left\|\boldsymbol{u}-\boldsymbol{u}_{h}\right\|_{\boldsymbol{V}}+\alpha_{1}^{\frac{1}{2}}\left\|\dot{\boldsymbol{d}}-\dot{\boldsymbol{d}}_{h}\right\|_{\mathrm{e}}+\left|p_{h}\right|_{s_{h}} \lesssim\left\|\boldsymbol{u}-\hat{\boldsymbol{v}}_{h}^{\mathrm{f}}\right\|_{\boldsymbol{V}}+\alpha_{1}^{\frac{1}{2}}\left\|\dot{\boldsymbol{d}}-\hat{\boldsymbol{v}}_{h}^{\mathrm{s}}\right\|_{\mathrm{e}}+\left\|\hat{p}_{h}\right\|_{Q}+\left|\hat{p}_{h}\right|_{s_{h}} .
$$

It remains to have a control on $\left\|p_{h}\right\|_{0, \Omega^{\mathrm{f}}}$. Owing to the generalized inf-sup condition of Lemma 9 , there exists $\left(\boldsymbol{z}_{h}^{\mathrm{f}}, \boldsymbol{z}_{h}^{\mathrm{s}}\right) \in \boldsymbol{W}_{h}^{\mathrm{f}} \backslash\{\mathbf{0}\}$ such that

$$
\begin{aligned}
\left\|p_{h}-\hat{p}_{h}\right\|_{Q} & \lesssim\left|p_{h}-\hat{p}_{h}\right|_{s_{h}}+\frac{b\left(p_{h}-\hat{p}_{h}, \boldsymbol{z}_{h}^{\mathrm{f}}\right)}{\left\|\boldsymbol{z}_{h}^{\mathrm{f}}\right\|_{\boldsymbol{V}}+\alpha_{1}^{\frac{1}{2}}\left\|\boldsymbol{z}_{h}^{\mathrm{s}}\right\|_{\mathrm{e}}} \\
& \lesssim\left|p_{h}-\hat{p}_{h}\right|_{s_{h}}+\frac{a\left(\boldsymbol{u}-\boldsymbol{u}_{h}, \boldsymbol{z}_{h}^{\mathrm{f}}\right)+\alpha_{1} a^{\mathrm{e}}\left(\dot{\boldsymbol{d}}-\dot{\boldsymbol{d}}_{h}, \boldsymbol{z}_{h}^{\mathrm{s}}\right)-b\left(\hat{p}_{h}, \boldsymbol{z}_{h}^{\mathrm{f}}\right)}{\left\|\boldsymbol{z}_{h}^{\mathrm{f}}\right\|_{\boldsymbol{V}}+\alpha_{1}^{\frac{1}{2}}\left\|\boldsymbol{z}_{h}^{\mathrm{s}}\right\|_{\mathrm{e}}} \\
& \lesssim\left|p_{h}-\hat{p}_{h}\right|_{s_{h}}+\left\|\boldsymbol{u}-\boldsymbol{u}_{h}\right\|_{\boldsymbol{V}}+\alpha_{1}^{\frac{1}{2}}\left\|\dot{\boldsymbol{d}}-\dot{\boldsymbol{d}}_{h}\right\|_{\mathrm{e}}+\left\|\hat{p}_{h}\right\|_{Q} .
\end{aligned}
$$

Hence, owing to (84) and (85), we get

$$
\left\|p_{h}-\hat{p}_{h}\right\|_{Q} \lesssim\left\|\boldsymbol{u}-\hat{\boldsymbol{v}}_{h}^{\mathrm{f}}\right\|_{\boldsymbol{V}}+\alpha_{1}^{\frac{1}{2}}\left\|\dot{\boldsymbol{d}}-\hat{\boldsymbol{v}}_{h}^{\mathrm{s}}\right\|_{\mathrm{e}}+\left\|\hat{p}_{h}\right\|_{Q}+\left|\hat{p}_{h}\right|_{s_{h}} .
$$

We conclude the estimate for the pressure with the triangle inequality

$$
\left\|p_{h}\right\|_{Q} \leq\left\|p_{h}-\hat{p}_{h}\right\|_{Q}+\left\|\hat{p}_{h}\right\|_{Q}
$$

We conclude the proof by noting that the choice of $\left(\left(\hat{\boldsymbol{v}}_{h}^{\mathrm{f}}, \hat{\boldsymbol{v}}_{h}^{\mathrm{s}}\right), \hat{p}_{h}\right) \in \boldsymbol{Z}_{h}$ is arbitrary.

The next lemma shows that the constraint $\left(\left(\hat{\boldsymbol{v}}_{h}^{\mathrm{f}}, \hat{\boldsymbol{v}}_{h}^{\mathrm{s}}\right), \hat{p}_{h}\right) \in \boldsymbol{Z}_{h}$ can be relaxed in (83).

Lemma 11 Let $\left(\left(\boldsymbol{u}_{h}, \dot{\boldsymbol{d}}_{h}\right), p_{h}\right) \in \boldsymbol{W}_{h} \times Q_{h}$ be given by (40) and assume that div $\boldsymbol{u}=0$ in $\Omega^{\mathrm{f}}$. The following inequality holds:

$$
\begin{aligned}
\left\|\boldsymbol{u}-\boldsymbol{u}_{h}\right\|_{\boldsymbol{V}}+\alpha_{1}^{\frac{1}{2}} \| \dot{\boldsymbol{d}} & -\dot{\boldsymbol{d}}_{h}\left\|_{\mathrm{e}}+\right\| p_{h} \|_{Q}+\left|p_{h}\right|_{s_{h}} \\
& \lesssim \inf _{\left(\left(\boldsymbol{z}_{h}^{\mathrm{f}}, \boldsymbol{z}_{h}^{\mathrm{s}}\right), r_{h}\right) \in \boldsymbol{W}_{h} \times Q_{h}}\left\{\left\|\boldsymbol{u}-\boldsymbol{z}_{h}^{\mathrm{f}}\right\|_{\boldsymbol{V}}+\alpha_{1}^{\frac{1}{2}}\left\|\dot{\boldsymbol{d}}-\boldsymbol{z}_{h}^{\mathrm{s}}\right\|_{\mathrm{e}}+\left\|r_{h}\right\|_{Q}+\left|r_{h}\right|_{s_{h}}\right\} .
\end{aligned}
$$

Proof 11 Let $\left(\left(\boldsymbol{z}_{h}^{\mathrm{f}}, \boldsymbol{z}_{h}^{\mathrm{s}}\right), r_{h}\right)$ be given in $\boldsymbol{W}_{h} \times Q_{h}$. We denote by $\left(\left(\boldsymbol{x}_{h}^{\mathrm{f}}, \boldsymbol{x}_{h}^{\mathrm{s}}\right), y_{h}\right) \in \boldsymbol{W}_{h} \times Q_{h}$ the solution of the following auxiliary problem:

$$
\left\{\begin{aligned}
a\left(\boldsymbol{x}_{h}^{\mathrm{f}}, \boldsymbol{v}_{h}^{\mathrm{f}}\right)+\alpha_{1} a^{\mathrm{e}}\left(\boldsymbol{x}_{h}^{\mathrm{s}}, \boldsymbol{v}_{h}^{\mathrm{s}}\right)+b\left(y_{h}, \boldsymbol{v}_{h}^{\mathrm{f}}\right) & =0, \\
b\left(q_{h}, \boldsymbol{x}_{h}^{\mathrm{f}}\right)-s_{h}\left(y_{h}, q_{h}\right) & =b\left(q_{h}, \boldsymbol{u}-\boldsymbol{z}_{h}^{\mathrm{f}}\right)+s_{h}\left(r_{h}, q_{h}\right)
\end{aligned}\right.
$$

for all $\left(\left(\boldsymbol{v}_{h}^{\mathrm{f}}, \boldsymbol{v}_{h}^{\mathrm{s}}\right), q_{h}\right) \in \boldsymbol{W}_{h} \times Q_{h}$. The existence and uniqueness of the solution $\left(\left(\boldsymbol{x}_{h}^{\mathrm{f}}, \boldsymbol{x}_{h}^{\mathrm{s}}\right), y_{h}\right)$ results from Lemma 9. Since div $\boldsymbol{u}=0$ in $\Omega^{\mathrm{f}}$, the relation $(86)_{2}$ implies that $\left(\left(\boldsymbol{x}_{h}^{\mathrm{f}}+\boldsymbol{z}_{h}^{\mathrm{f}}, \boldsymbol{x}_{h}^{\mathrm{s}}+\boldsymbol{z}_{h}^{\mathrm{s}}\right), y_{h}+r_{h}\right) \in$ $Z_{h}$. Therefore,

$$
\begin{aligned}
& \quad \inf _{\left(\left(\hat{\boldsymbol{v}}_{h}^{\mathrm{f}}, \hat{\boldsymbol{v}}_{h}^{\mathrm{s}}\right), \hat{p}_{h}\right) \in \boldsymbol{Z}_{h}}\left\{\left\|\boldsymbol{u}-\hat{\boldsymbol{v}}_{h}^{\mathrm{f}}\right\|_{\boldsymbol{V}}+\alpha_{1}^{\frac{1}{2}}\left\|\dot{\boldsymbol{d}}-\hat{\boldsymbol{v}}_{h}^{\mathrm{s}}\right\|_{\mathrm{e}}+\left\|\hat{p}_{h}\right\|_{Q}+\left|\hat{p}_{h}\right|_{s_{h}}\right\} \\
& \leq\left\|\boldsymbol{u}-\left(\boldsymbol{x}_{h}^{\mathrm{f}}+\boldsymbol{z}_{h}^{\mathrm{f}}\right)\right\|_{\boldsymbol{V}}+\alpha_{1}^{\frac{1}{2}}\left\|\dot{\boldsymbol{d}}-\left(\boldsymbol{x}_{h}^{\mathrm{s}}+\boldsymbol{z}_{h}^{\mathrm{s}}\right)\right\|_{\mathrm{e}}+\left\|y_{h}+r_{h}\right\|_{Q}+\left|y_{h}+r_{h}\right|_{s_{h}} \\
& \leq\left\|\boldsymbol{u}-\boldsymbol{z}_{h}^{\mathrm{f}}\right\|_{\boldsymbol{V}}+\alpha_{1}^{\frac{1}{2}}\left\|\dot{\boldsymbol{d}}-\boldsymbol{z}_{h}^{\mathrm{s}}\right\|_{\mathrm{e}}+\left\|r_{h}\right\|_{Q}+\left|r_{h}\right|_{s_{h}}+\left\|\boldsymbol{x}_{h}^{\mathrm{f}}\right\|_{\boldsymbol{V}}+\alpha_{1}^{\frac{1}{2}}\left\|\boldsymbol{x}_{h}^{\mathrm{s}}\right\|_{\mathrm{e}}+\left\|y_{h}\right\|_{Q}+\left|y_{h}\right|_{s_{h}} .
\end{aligned}
$$


In order to control the last four terms, we take $\left(\boldsymbol{v}_{h}^{\mathrm{f}}, \boldsymbol{v}_{h}^{\mathrm{s}}\right)=\left(\boldsymbol{x}_{h}^{\mathrm{f}}, \boldsymbol{x}_{h}^{\mathrm{s}}\right)$ and $q_{h}=y_{h}$ in (86), which yields

$$
\left\|\boldsymbol{x}_{h}^{\mathrm{f}}\right\|_{\boldsymbol{V}}+\alpha_{1}^{\frac{1}{2}}\left\|\boldsymbol{x}_{h}^{\mathrm{s}}\right\|_{\mathrm{e}}+\left|y_{h}\right|_{s_{h}} \lesssim\left\|\boldsymbol{u}-\boldsymbol{z}_{h}^{\mathrm{f}}\right\|_{\boldsymbol{V}}+\left\|y_{h}\right\|_{Q}+\left|r_{h}\right|_{s_{h}} .
$$

Furthermore, using the generalized inf-sup condition of Lemma 9, we infer that

$$
\left\|y_{h}\right\|_{Q} \lesssim\left|y_{h}\right|_{s_{h}}+\left\|\boldsymbol{x}_{h}^{\mathrm{f}}\right\|_{\boldsymbol{V}}+\alpha_{1}^{\frac{1}{2}}\left\|\boldsymbol{x}_{h}^{\mathrm{s}}\right\|_{\mathrm{e}} .
$$

We hence conclude by inserting (88) and (89) into (87) and using the arbitrariness of $\left(\left(\boldsymbol{z}_{h}^{\mathrm{f}}, \boldsymbol{z}_{h}^{\mathrm{s}}\right), r_{h}\right) \in$ $\boldsymbol{W}_{h} \times Q_{h}$.

Finally, the error estimate (43) follows from Lemma 11, by setting $r_{h}=0$ and taking $\boldsymbol{z}_{h}^{\mathrm{f}}, \boldsymbol{z}_{h}^{\mathrm{s}}$ as the Lagrange interpolants of $\boldsymbol{u}, \dot{\boldsymbol{d}}$, respectively, and then using standard interpolation theory. Note that, with this choice we do have $\left(\boldsymbol{z}_{h}^{\mathrm{f}}, \boldsymbol{z}_{h}^{\mathrm{s}}\right) \in \boldsymbol{W}_{h}$, since $(\boldsymbol{u}, \dot{\boldsymbol{d}}) \in \boldsymbol{W}$.

\section{References}

[1] M. Astorino and C. Grandmont. Convergence analysis of a projection semi-implicit coupling scheme for fluid-structure interaction problems. Numer. Math., 116:721-767, 2010.

[2] S. Badia, F. Nobile, and C. Vergara. Fluid-structure partitioned procedures based on Robin transmission conditions. J. Comp. Phys., 227:7027-7051, 2008.

[3] S. Badia, A. Quaini, and A. Quarteroni. Splitting methods based on algebraic factorization for fluid-structure interaction. SIAM J. Sci. Comput., 30(4):1778-1805, 2008.

[4] J.W. Banks, W.D. Henshaw, and D.W. Schwendeman. An analysis of a new stable partitioned algorithm for FSI problems. Part I: Incompressible flow and elastic solids. J. Comput. Phys., 269:108-137, 2014.

[5] S.C. Brenner and L.R. Scott. The mathematical theory of finite element methods, volume 15 of Texts in Applied Mathematics. Springer, New York, 2008.

[6] F. Brezzi and J. Pitkäranta. On the stabilization of finite element approximations of the Stokes equations. volume 10 of Notes Numer. Fluid Mech., pages 11-19. Vieweg, 1984.

[7] M. Bukac, S. Canic, R. Glowinski, B. Muha, and A. Quaini. A modular, operator-splitting scheme for fluid-structure interaction problems with thick structures. Int. J. Numer. Meth. Fluids., 74(8):577-604, 2014.

[8] M. Bukac, S. Canic, R. Glowinski, J. Tambaca, and A. Quaini. Fluid-structure interaction in blood flow capturing non-zero longitudinal structure displacement. J. Comp. Phys., 235:515-541, 2013.

[9] E. Burman and M.A. Fernández. Galerkin finite element methods with symmetric pressure stabilization for the transient Stokes equations: stability and convergence analysis. SIAM J. Numer. Anal., 47(1):409-439, 2008.

[10] E. Burman and M.A. Fernández. Stabilization of explicit coupling in fluid-structure interaction involving fluid incompressibility. Comput. Methods Appl. Mech. Engrg., 198(5-8):766$784,2009$. 
[11] E. Burman and M.A. Fernández. Explicit strategies for incompressible fluid-structure interaction problems: Nitsche type mortaring versus Robin-Robin coupling. Internat. J. Numer. Methods Engrg., 97(10):739-758, 2014.

[12] E. Burman, M.A. Fernández, and P. Hansbo. Continuous interior penalty finite element method for Oseen's equations. SIAM J. Numer. Anal., 44(3):1248-1274, 2006.

[13] P. Causin, J.-F. Gerbeau, and F. Nobile. Added-mass effect in the design of partitioned algorithms for fluid-structure problems. Comput. Methods Appl. Mech. Engrg., 194(4244):4506-4527, 2005.

[14] V. Domínguez and F.-J. Sayas. Stability of discrete liftings. C. R. Math. Acad. Sci. Paris, 337(12):805-808, 2003.

[15] Q. Du, M. D. Gunzburger, L. S. Hou, and J. Lee. Analysis of a linear fluid-structure interaction problem. Discrete Contin. Dyn. Syst., 9(3):633-650, 2003.

[16] Q. Du, M. D. Gunzburger, L. S. Hou, and J. Lee. Semidiscrete finite element approximations of a linear fluid-structure interaction problem. SIAM J. Numer. Anal., 42(1):1-29 (electronic), 2004.

[17] M.A. Fernández. Incremental displacement-correction schemes for incompressible fluidstructure interaction: stability and convergence analysis. Numer. Math., 123(1):21-65, 2013.

[18] M.A. Fernández, J.F. Gerbeau, and C. Grandmont. A projection semi-implicit scheme for the coupling of an elastic structure with an incompressible fluid. Int. J. Num. Meth. Engrg., 69(4):794-821, 2007.

[19] M.A. Fernández and J. Mullaert. Displacement-velocity correction schemes for incompressible fluid-structure interaction. C. R. Math. Acad. Sci. Paris, 349(17-18):1011-1015, 2011.

[20] M.A. Fernández, J. Mullaert, and M. Vidrascu. Explicit Robin-Neumann schemes for the coupling of incompressible fluids with thin-walled structures. Comput. Methods Appl. Mech. Engrg., 267:566-593, 2013.

[21] M.A. Fernández, J. Mullaert, and M. Vidrascu. Generalized Robin-Neumann explicit coupling schemes for incompressible fluid-structure interaction: stability analysis and numerics. Internat. J. Numer. Methods Engrg., 101(3):199-229, 2015.

[22] C.A. Figueroa, I.E. Vignon-Clementel, K.E. Jansen, T.J.R. Hughes, and C.A. Taylor. A coupled momentum method for modeling blood flow in three-dimensional deformable arteries. Comput. Methods Appl. Mech. Engrg., 195(41-43):5685-5706, 2006.

[23] C. Förster, W.A. Wall, and E. Ramm. Artificial added mass instabilities in sequential staggered coupling of nonlinear structures and incompressible viscous flows. Comput. Methods Appl. Mech. Engrg., 196(7):1278-1293, 2007.

[24] G. Guidoboni, R. Glowinski, N. Cavallini, and S. Canic. Stable loosely-coupled-type algorithm for fluid-structure interaction in blood flow. J. Comp. Phys., 228(18):6916-6937, 2009.

[25] M.D. Gunzburger and S.L. Hou. Treating inhomogeneous essential boundary conditions in finite element methods and the calculation of boundary stresses. SIAM J. Numer. Anal., 29(2):390-424, 1992. 
[26] P. Hansbo. Nitsche's method for interface problems in computational mechanics. GAMMMitt., 28(2):183-206, 2005.

[27] F. Hecht. New development in FreeFem++. J. Numer. Math., 20(3-4):251-265, 2012.

[28] P. Joly. Variational methods for time-dependent wave propagation problems. In M. Ainsworth, P. Davies, D. Duncan, B. Rynne, and P. Martin, editors, Topics in Computational Wave Propagation, volume 31 of Lecture Notes in Computational Science and Engineering, pages 201-264. Springer Berlin Heidelberg, 2003.

[29] P. Le Tallec and S. Mani. Numerical analysis of a linearised fluid-structure interaction problem. Numer. Math., 87(2):317-354, 2000.

[30] M. Lukacova, G. Rusnakova, and A. Hundertmark. Kinematic splitting algorithm for fluidstructure interaction in hemodynamics. Comput. Methods Appl. Mech. Engrg., 265(1):83$106,2013$.

[31] F. Nobile and C. Vergara. An effective fluid-structure interaction formulation for vascular dynamics by generalized Robin conditions. SIAM J. Sci. Comput., 30(2):731-763, 2008.

[32] A. Quaini and A. Quarteroni. A semi-implicit approach for fluid-structure interaction based on an algebraic fractional step method. Math. Models Methods Appl. Sci., 17(6):957-983, 2007.

[33] V. Thomée. Galerkin finite element methods for parabolic problems. Springer, 2006. 


\section{Contents}

1 Introduction $\quad 3$

2 Problem setting $\quad 4$

3 Numerical methods $\quad 5$

3.1 Spatial semi-discrete approximation . . . . . . . . . . . . . . . . 6

3.2 Time discretization: coupling schemes . . . . . . . . . . . . . . . . . . 7

3.2.1 Semi-implicit coupling schemes . . . . . . . . . . . . . . . 7

3.2.2 Generalized Robin-Neumann explicit coupling schemes . . . . . . . . . . 8

3.2.3 Alternative splitting scheme . . . . . . . . . . . . . . 11

4 Convergence analysis $\quad 12$

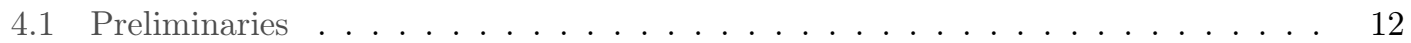

4.2 Projection operators . . . . . . . . . . . . . . . . . . . . 13

4.3 Error decomposition . . . . . . . . . . . . . . . . . . . 15

4.4 Discrete error estimation . . . . . . . . . . . . . . . . . . . . . . . . . . . . . . . . . . . . . . .

4.5 Stability of the discrete viscoelastic operator . . . . . . . . . . . . . . . . 25

4.6 A priori error estimates . . . . . . . . . . . . . . . . 27

$\begin{array}{llr}5 & \text { Numerical experiments } & \mathbf{2 8}\end{array}$

6 Conclusion $\quad 29$

$\begin{array}{lr}\text { A Proof of Lemma 3 } & 30\end{array}$ 


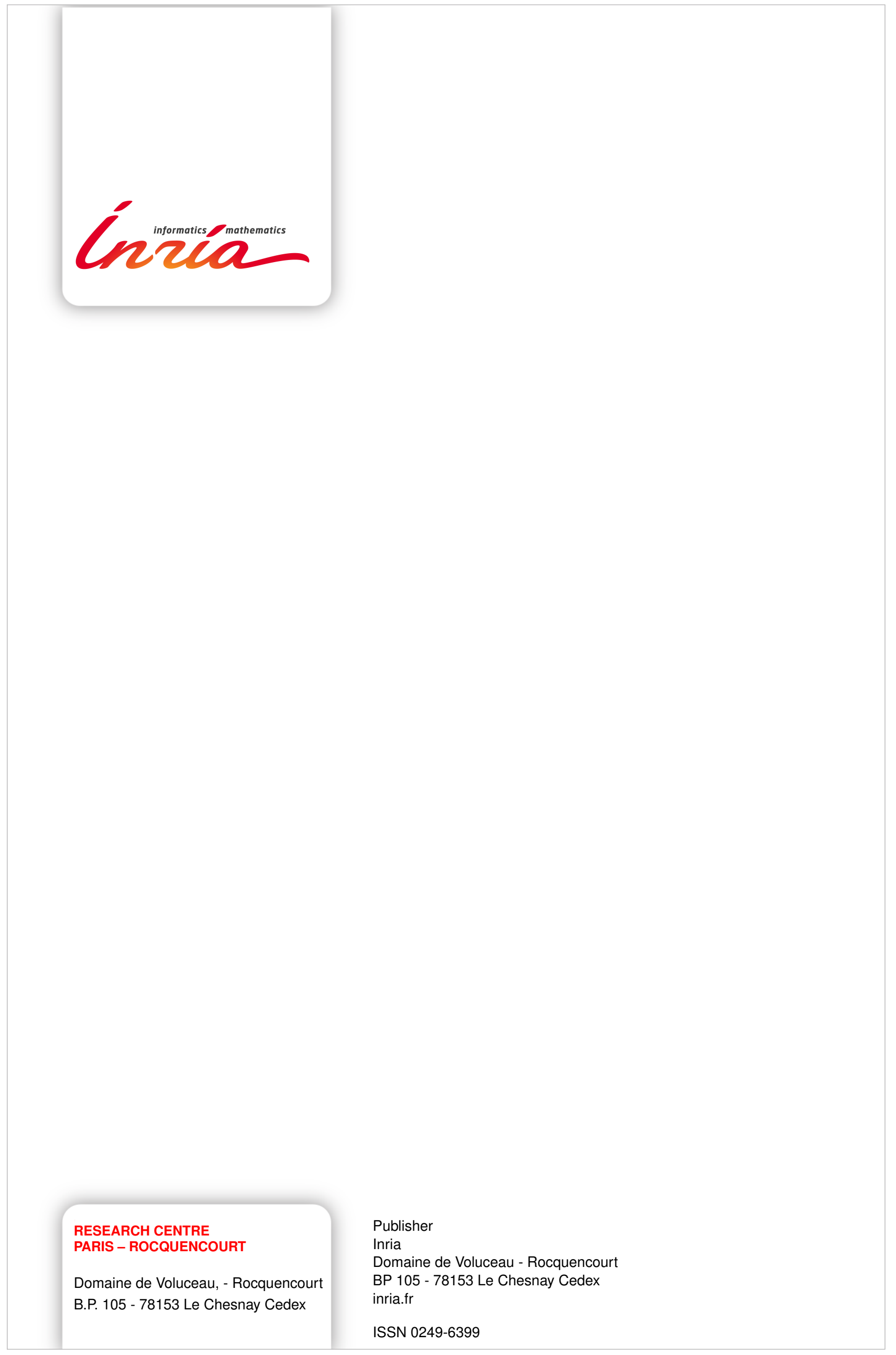

